SLAC-PUB-13383

September 2008

\title{
Interaction-Point Phase-Space Characterization using Single-Beam and Luminous-Region Measurements at PEP-II *
}

\author{
W. Kozanecki, CEA/IRFU/SPP, F-91191 Gif-sur-Yvette, France \\ A. J. Bevan, Queen Mary, University of London Dept of Physics, Mile End Road, London, E1 4NS, UK \\ B. F. Viaud `ं, Université de Montréal, Montréal, Québec, Canada H3C 3J7 \\ Y. Cai, A. S. Fisher, C. O'Grady, B. Lindquist, A. Roodman, J. M. Thompson, M. Weaver, Stanford \\ Linear Accelerator Center, Stanford CA 94309, U.S.A.
}

We present an extensive experimental characterization of the $e^{ \pm}$phase space at the interaction point of the SLAC PEP-II $B$-Factory, that combines a detailed mapping of luminous-region observables using the $B A B A R$ detector, with stored-beam measurements by accelerator techniques.

\section{Introduction}

Measuring the beam parameters at the interaction point (IP) of $e^{+} e^{-}$colliders has historically been both essential and extremely challenging for accelerator builders. Exploiting the shape of the event-vertex distribution to measure bunch length, emittance and IP $\beta$-function was pioneered by Cinabro et al. [1][2] using the CLEO detector at CESR; a similar approach was later exploited at the Tevatron [3]. The advent of highluminosity $e^{+} e^{-}$Factories opened opportunities for more refined measurements, at the price however of an increased complexity: in a two-ring collider, the phase space and IP optical functions of the two beams are no longer constrained to be equal. At PEP-II [4], the very large usable event rate, combined with the performance of the $B A B A R$ silicon vertex tracker [5] and with advances in data acquisition and on-line processing capability, made it possible to reconstruct in real time the spatial and transverse-momentum distributions of $e^{+} e^{-}, \mu^{+} \mu^{-}$final states, which reflect the phase-space distributions of the colliding $e^{ \pm}$. This allowed to provide feedback to the accelerator, on the time scale of a few tens of minutes, on the position, orientation, size and shape of the luminous region.

\footnotetext{
*Submitted to NIM-A for publication. Work supported in part by DOE Contract DE-AC02-76SFO0515

${ }^{\dagger}$ now at Laboratoire de l'Accélérateur Linéaire, Orsay, France
}

In this paper, we present an extensive characterization of the $e^{ \pm}$phase space at the PEP-II IP, that combines (Fig. 1) a detailed mapping of luminous-region observables using $e^{+} e^{-} \rightarrow$ $\mu^{+} \mu^{-}, e^{+} e^{-}$events, with single-beam measurements by conventional accelerator techniques. The $B$-Factory and the relevant aspects of the BABAR detector are described in Sec. 2. The formalism relating experimental observables to beam parameters is presented in Sec. 3. On-line event reconstruction allows real-time monitoring of IP position and beam angles, horizontal and longitudinal luminous sizes, vertical $\beta$-function and angular divergences (Sec. 4). The spatial variation of the luminosity (Secs. 5 and 6), and the transverse-boost distribution (Sec. 7) of the colliding $e^{ \pm}$provide ready access to simple combinations of the spatial and angular variables describing the beam matrices near the IP. The specific luminosity (which is proportional to the inverse product of the overlap IP beam sizes) is continuously monitored, while transverse spot sizes are measured in the two rings using synchrotronlight monitors and extrapolated to the IP using measured lattice functions (Sec. 8). A combined offline analysis of these measurements provides constraints on the emittances, horizontal and vertical spot sizes, $\beta$ functions and bunch lengths of both beams at the IP during physics data-taking (Sec. 9). 


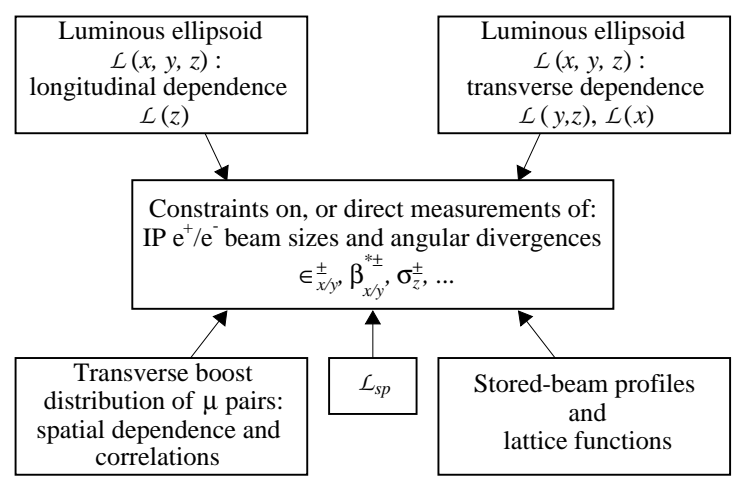

Figure 1. Conceptual flow chart of the combined phase-space analysis at the PEP-II IP.

\section{Experimental Context}

\subsection{The SLAC B-Factory}

PEP-II $[4,6]$ is an asymmetric-energy collider designed to operate at the $\Upsilon(4 S)$ resonance with the $e^{+} e^{-}$center-of-mass boosted by $\beta \gamma=0.56$. It consists of two rings, stacked vertically in a $2.2 \mathrm{~km}$ tunnel. Positrons from the SLAC linac are continuously injected on-energy and accumulated in the low-energy ring (LER), as are electrons in the high-energy ring (HER). The beams collide head-on in the middle of the BABAR experiment, and are separated magnetically by a combination of permanent-magnet dipoles and offaxis quadrupoles. Beam parameters at the IP are listed in Table 1 . The history of the B-Factory luminosity performance is summarized in Fig. 2.

$B A B A R$ [5] is a general-purpose detector for particle physics, consisting of multiple subsystems for the detection and identification of charged and neutral particles. The detector is longitudinally asymmetric, commensurate with the difference between the $e^{-}$and $e^{+}$beam energies. The Silicon Vertex Tracker (SVT) is located closest to the beam pipe. Charged-particle tracking is performed by the SVT in conjunction with the surrounding drift chamber (DCH). Outside the $\mathrm{DCH}$ are the quartz bars of the Cerenkov-light detector (DIRC), followed by the thallium-doped
Table 1

Design beam parameters [4] at the PEP-II IP. The numbers in parentheses are the best-achieved values (not necessarily simultaneously).

\begin{tabular}{|c|cc|c|}
\hline & LER $\left(e^{+}\right)$ & HER $\left(e^{-}\right)$ & Units \\
\hline Energy & 3.1 & 9.0 & $\mathrm{GeV}$ \\
$\epsilon_{x} / \epsilon_{y}$ & $64 / 2.6$ & $48 / 1.9$ & nm-rad \\
$\beta_{x}^{*} / \beta_{y}^{*}$ & $37.5 / 1.5$ & $50 / 2.0$ & $\mathrm{~cm}$ \\
$\sigma_{x}^{*} / \sigma_{y}^{*}$ & $155 / 6.2$ & $155 / 6.2$ & $\mu \mathrm{m}$ \\
$\sigma_{x}^{\prime} / \sigma_{y}^{\prime}$ & $0.4 / 0.4$ & $0.3 / 0.3$ & $\mathrm{mrad}$ \\
$\sigma_{z}$ & 1.0 & 1.0 & $\mathrm{~cm}$ \\
\hline Current & $2140(3213)$ & $990(2069)$ & $\mathrm{mA}$ \\
$N_{\text {bunch }}$ & \multicolumn{2}{|c|}{$1658(1726)$} & \\
$\mathcal{L}$ & $3(12) \times 10^{33}$ & $\mathrm{~cm}^{-2} \mathrm{~s}^{-1}$ \\
\hline
\end{tabular}

cesium-iodide crystals that form the electromagnetic calorimeter (EMC). The EMC measures electromagnetic showers, providing sensitivity to neutral particles and discrimination between electrons and muons. These detectors are immersed in a $1.5 \mathrm{~T}$ solenoidal magnetic field. The outermost detector is the Instrumented Flux Return, composed of steel plates interleaved with gaseous detectors, for the detection and identification of muons and $K_{L}^{0}$.

The fast luminosity monitor [7] consists of a fused-silica Čerenkov hodoscope with photomultiplier-tube readout, that counts photons emitted at the IP, through the radiativeBhabha process $\left(e^{+} e^{-} \rightarrow e^{+} e^{-} \gamma\right)$, collinear with the incoming positrons. It is located $8 \mathrm{~m}$ downstream of the IP and provides bunch-by-bunch and total luminosity measurements at the rate of a few Hz. Its absolute normalization is pegged to the luminosity measured, on a slower time scale, using wider-angle Bhabhas reconstructed in BABAR.

\subsection{The BABAR Tracking System}

The BABAR charged-particle tracking system, the performance of which is crucial to many of the results in this paper, was designed for precision vertexing, as well as for excellent momentum resolution over the entire $B A B A R$ fiducial volume. This is achieved by combining SVT measure- 

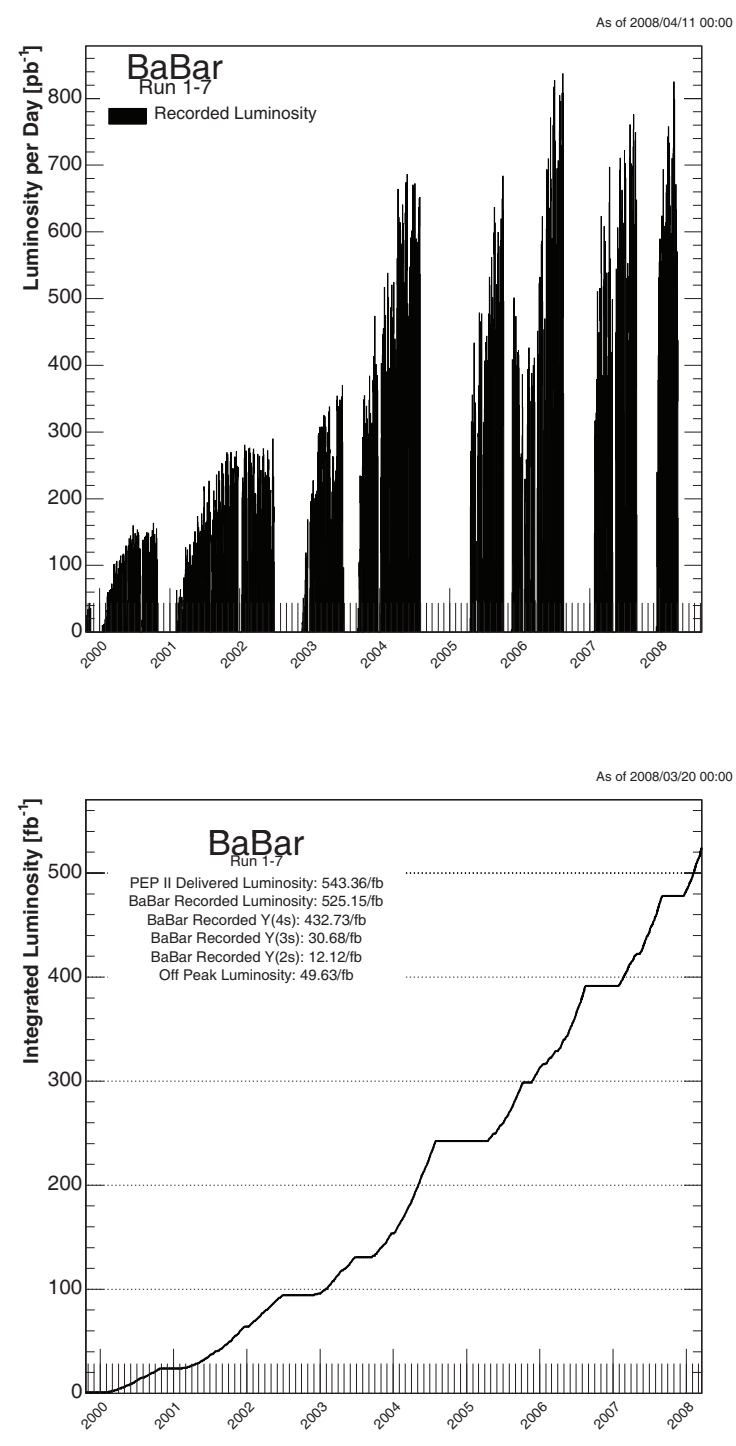

Figure 2. Daily integrated luminosity (top) and cumulative integrated luminosity (bottom) for PEP-II and BABAR.

ments, which allow stand-alone precision tracking close to the beam line, with those of the Drift Chamber, which provide a longer lever arm for momentum measurement. Signals from the DCH are also used to find tracks for the $B A B A R$ trigger system.

\subsubsection{The Silicon Vertex Tracker}

The SVT consists of five layers of $300 \mu \mathrm{m}$ thick double-sided silicon-strip detectors that provide both $z$ and $r-\phi$ coordinate measurements as close as possible to the primary event vertex. A custom application-specific integrated circuit (ASIC) reads out the time-over-threshold for each strip.

The BABAR beam-pipe has an inner radius of $2.5 \mathrm{~cm}$ and consists of two cylinders of Be with a cooling-water channel; it is a total of $0.9 \% X_{0}$ thick. The inner three layers of the SVT consist of six modules arranged azimuthally around the beam pipe, at radii of $3.2,4.0$, and $5.4 \mathrm{~cm}$. The two outer layers consist of a barrel portion plus an angled arch to increase the coverage in polar angle $\theta$ while minimizing the amount of multiple scattering. While the inner three layers are the most critical for vertex resolution, the entire five-layer device enables tracks to be found in the SVT alone, allowing redundant track finding between the SVT and DCH. The readout pitch ranges from $50 \mu \mathrm{m}$ to $220 \mu \mathrm{m}$, depending on the layer and view. For the $100 \mu \mathrm{m}$ pitch detectors, the single hit resolution is better than $20 \mu \mathrm{m}$ at normal incidence. The silicon strip detectors are mounted on very-low-mass carbon-fiber supports, so at normal incidence the SVT as a whole is $2.3 \% X_{0}$ thick. The entire SVT is mounted in a carbon-fiber support tube $0.5 \% X_{0}$ thick.

\subsubsection{The Drift Chamber}

The DCH consists of 40 layers arranged in 10 super-layers. There are four axial and six stereo super-layers, balancing the requirements of track finding and measurements in the $r-z$ view. The DCH uses an 80:20 mixture of helium and isobutane, a gas that provides nearly minimum charge collection time, while also minimizing the total radiative thickness of the detector. The inner wall of the $\mathrm{DCH}$ is $0.3 \% X_{0}$ thick and the gas and wires comprise only $0.16 \% X_{0}$. The DCH has hexagonal cells, arranged in a close-packed pattern, within each super-layer. The single hit resolution is better than $100 \mu \mathrm{m}$ in the interior of each cell. Custom ASICs digitize the drift time 
and charge on each wire.

\subsubsection{Tracking And Vertexing Perfor- mance}

Track finding is done independently in the SVT and $\mathrm{DCH}$, and the resulting tracks are combined whenever possible. Tracks are fitted with a Kalman-filter algorithm [8] that incorporates detailed information about the magnetic field and detector material. The Kalman filter starts at the outside of the DCH and, for each step, adds measured hits with appropriate errors, the integral of the magnetic field traversed, and multiple scattering from detector material. Extrapolated to the beam, each track fit has a covariance matrix that includes the detector resolution for each SVT and DCH hit on the track, as well as the estimated multiple scattering from a detailed detector model. Thus the covariance matrix is an accurate representation of the errors for each track.

The relative alignment of the SVT and DCH is critical in obtaining bias-free measurements of decay vertices and particle trajectories. This alignment is updated approximately once per hour by minimizing the differences between tracks reconstructed separately in the SVT and DCH. The SVT is treated as a rigid body in this "global" alignment. An additional "local" alignment is critical for the luminous-region studies described in Secs. 4-7, that depend on an accurate alignment of the SVT at the individual-sensor level. The SVT alignment is performed [9] with pairs of tracks from Bhabha and muon-pair events, selected to be sensitive to local misalignments. The free parameters in the local alignment fit are the position and orientation of each of the 340 SVT sensors, as well as a piece-wise linear bend along the long axis of each sensor. These alignment constants are determined from a combination of track residuals and optical survey constraints. Particular attention was paid to prevent global distortions. The alignment procedure was determined to be accurate at the level of $5 \mu \mathrm{m}$ or better. Because of the complexity of the local alignment, it is performed more rarely (roughly once per month).

The track momentum resolution is parameter- ized as: $\sigma\left(p_{T}\right) / p_{T}=(0.13 \pm 0.01) \% \cdot p_{T}+(0.45 \pm$ $0.03) \%$ [5]. The vertex resolution for high momentum tracks is $23 \mu \mathrm{m}$ on the average, with a range of 15 to $30 \mu \mathrm{m}$ and above, depending on the region of the SVT traversed.

\section{Experimental Observables and IP Beam Parameters}

\subsection{Overview}

The notation used to describe the single-beam phase-space at the IP is defined in Sec. 3.2 below. The spatial distribution of the luminosity can be quantitatively described in terms of the parameters of the luminous ellipsoid (Sec. 3.3), and experimentally characterized using the distribution of event vertices (Sec. 4). Of particular interest for extracting IP parameters is the hourglass effect, discussed in Sec. 3.4. This formalism is applied to the experimental study of the longitudinal luminosity distribution in Sec. 5; it is then used, in Sec. 6, to extract the vertical emittance and IP $\beta$-function from the measured longitudinal dependence of the vertical luminous size. Both the spatial and the angular distributions of the incoming electrons and positrons determine the transverse-boost distribution of $\mu^{+} \mu^{-}$pairs: the formalism is presented in Sec. 3.5, and the corresponding experimental studies in Sec. 7. Singlebeam parameters can in principle be determined by a combination of orbit-based lattice diagnostics (Sec. 3.6) and synchrotron-light beam-profile measurements (Sec. 3.7); the experimental data and their interpretation in terms of IP parameters are presented in Sec. 8.

Except where specified otherwise, the following assumptions apply throughout this paper.

- $x$-y coupling is small enough to be neglected, both around the collider rings and within the BABAR solenoid. This assumption will be relaxed in Secs. 3.7 and 8, and in part of Sec. 9.

- IP dispersion and angular dispersion are considered negligible.

- The transverse $e^{ \pm}$densities are Gaussian, implying that beam-beam induced distortions and lattice non-linearities are assumed 
to remain negligible. The potential impact of the beam-beam interaction on specific measurements will be considered in the corresponding chapters.

- RF-impedance effects are supposed to be weak enough for the longitudinal $e^{ \pm}$densities to remain Gaussian within measurement errors. The validity of this simplifying assumption is tested in Sec. 5.1.

\subsection{Single-beam IP Parameters}

If one assumes only that the beams are Gaussian, the $e^{ \pm}$particle density distribution can be written

$$
\begin{gathered}
\rho\left(x, x^{\prime}, y, y^{\prime}, z, t\right)=\frac{N}{\sqrt{(2 \pi)^{5} \operatorname{det} \sigma_{T}} \sigma_{z}} \times \\
\exp \left[-\frac{(z \pm c t)^{2}}{2 \sigma_{z}^{2}}\right] \times \exp \left[-\frac{1}{2} \overrightarrow{\delta r}^{T} \sigma_{T}^{-1} \overrightarrow{\delta r}\right]
\end{gathered}
$$

with

$$
\begin{aligned}
\overrightarrow{\delta r} & =\left(\begin{array}{c}
\delta x \\
\delta x^{\prime} \\
\delta y \\
\delta y^{\prime}
\end{array}\right), \\
\sigma_{T} & =\left(\begin{array}{cccc}
\sigma_{x}^{2} & \sigma_{x x^{\prime}} & \sigma_{x y} & \sigma_{x y^{\prime}} \\
\cdots & \sigma_{x^{\prime}}^{2} & \sigma_{x^{\prime} y} & \sigma_{x^{\prime} y^{\prime}} \\
\cdots & \cdots & \sigma_{y}^{2} & \sigma_{y y^{\prime}} \\
\cdots & \cdots & \cdots & \sigma_{y^{\prime}}^{2}
\end{array}\right) .
\end{aligned}
$$

Here $x, y, z, x^{\prime}, y^{\prime}$ are the spatial coordinates and angles of a beam particle at time $t$, the vector $\overrightarrow{\delta r}$ is the deviation of the particle trajectory from the closed orbit, $\sigma_{z}$ is the bunch length and $\sigma_{T}$ the beam matrix. In the case of no transverse coupling the latter simplifies to

$\sigma_{T}=\left(\begin{array}{cc}\Lambda_{x} & 0 \\ 0 & \Lambda_{y}\end{array}\right)$

where $i=x$ or $y$,

$$
\begin{aligned}
\Lambda_{i} & =\epsilon_{i}\left(\begin{array}{cc}
\beta_{i} & -\alpha_{i} \\
-\alpha_{i} & \gamma_{i}
\end{array}\right), \\
\alpha_{x, y} & =-\frac{1}{2} \frac{\delta \beta_{x, y}}{\delta s},
\end{aligned}
$$

and

$$
\gamma_{x, y}=\frac{1+\alpha_{x, y}^{2}}{\beta_{x, y}} .
$$

Between the optical waist (nominally located at the IP) and the first focusing element, and neglecting the $B A B A R$ solenoid, these simplify to

$\beta_{y}=\beta_{y}^{*}+\frac{\left(z-z_{y}^{w}\right)^{2}}{\beta_{y}^{*}}$

$\alpha_{y}=-\frac{z-z_{y}^{w}}{\beta_{y}^{*}}$

and similarly for $x$. The parameters determining the transverse distributions at the IP are, for each beam, the $x$ and $y$ emittances $\epsilon$, IP $\beta$-functions $\beta^{*}$ and waist locations $z^{w}$. The individual transverse beam sizes $\sigma_{i b}(i=x, y$ and $b=+,-)$ are given by:

$\sigma_{i b}=\sqrt{\epsilon_{i b} \beta_{i b}}$.

These are not directly measurable at the IP, but can be inferred from synchrotron-light beamprofile measurements elsewhere in the rings (Sec. 3.7), provided the lattice functions are known with sufficient accuracy.

\subsection{The Luminous Ellipsoid}

Under the assumptions in Sec. 3.1 above, the particle distribution in a bunch becomes

$\rho_{b}(x, y, z, t)=\frac{N_{b}}{\sqrt{(2 \pi)^{3}} \sigma_{x b} \sigma_{y b} \sigma_{z b}} \times$

$\exp \left[-\frac{\left(x-\bar{x}_{b}\right)^{2}}{2 \sigma_{x b}^{2}}-\frac{\left(y-\bar{y}_{b}\right)^{2}}{2 \sigma_{y b}^{2}}-\frac{(z-c t)^{2}}{2 \sigma_{z b}^{2}}\right]$

where $b=+$ and $b=-$ are associated with the LER and HER, respectively. $N_{b}$ is the number of particles in the bunch, the $\sigma_{j b}(j=x, y, z)$ are the transverse and longitudinal stored-beam sizes, and $\bar{x}_{b}$ and $\bar{y}_{b}$ are the transverse bunch centroids. The three-dimensional spatial luminosity distribution $\mathcal{L}(x, y, z)$, also known as the luminous ellipsoid, is determined by the timeintegrated product of the overlapping particle densities of the two colliding beams.

In general, luminosity-weighted observables can be calculated by taking the appropriate moment of the product of beam particle densities. 
For example, the vertical luminous centroid is given by

$\bar{y}_{\mathcal{L}}(z)=\frac{\int y \rho_{+} \rho_{-}}{\int \rho_{+} \rho_{-}}$

where the integral runs over $x, y, x_{+}^{\prime}, x_{-}^{\prime}, y_{+}^{\prime}$, $y_{-}^{\prime}$ and $t$. Similarly, the vertical (or $y-z$ ) $l u$ minous tilt is defined as $\bar{y}_{\mathcal{L}}=\frac{\delta \bar{y}_{\mathcal{L}}}{\delta z}$. Equivalent expressions hold for the horizontal centroid and luminous tilt, and for the longitudinal centroid. These parameters define the position and orientation of the luminous ellipsoid; they are continuously monitored on-line (Sec. 4), and must be taken into account when analyzing the long-term history of IP parameters (Secs. 5-7).

For head-on collisions (no relative transverse offsets, negligible crossing angles), the longitudinal luminosity distribution is given by

$$
\begin{aligned}
\frac{d \mathcal{L}}{d z} & =2 c \int \rho_{+} \rho_{-} d t d x d y \\
& =\frac{2 N_{-} N_{+}}{\sqrt{(2 \pi)^{3}} \Sigma_{z} \Sigma_{x} \Sigma_{y}} \exp \left(-\frac{\left(z-z_{c}\right)^{2}}{2\left(\Sigma_{z} / 2\right)^{2}}\right)
\end{aligned}
$$

where $z_{c}$ is the longitudinal location where the bunches collide and

$\Sigma_{j}=\sqrt{{\sigma_{j-}{ }^{2}+\sigma_{j+}{ }^{2}}}$

are the convoluted beam sizes. The longitudinal convoluted beam size $\Sigma_{z}$ can be extracted, together with the effective vertical IP $\beta$ function $\beta_{y, \text { eff }}^{*}$, from the longitudinal luminosity distribution; that formalism is outlined in Sec. 3.4. The transverse convoluted beam sizes $\Sigma_{x}, \Sigma_{y}$ can be measured by beam-beam scans [10]-[12], but only at small bunch currents, i.e. when the beambeam parameters are low enough.

A related - albeit distinct - measure of transverse phase space is supplied by the vertical $l u$ minous size $\sigma_{y \mathcal{L}}$, defined by

$\left(\sigma_{y \mathcal{L}}\right)^{2}=\overline{y^{2}}{ }_{\mathcal{L}}(z)=\frac{\int y^{2} \rho_{+} \rho_{-}}{\int \rho_{+} \rho_{-}}$,

which is related to the stored-beam sizes by

$\sigma_{y \mathcal{L}}=\left(\frac{1}{\sigma_{y-}{ }^{2}}+\frac{1}{\sigma_{y+}{ }^{2}}\right)^{-1 / 2}$, with equivalent expressions for $\sigma_{x \mathcal{L}}$. These two parameters describe the transverse shape of the luminous ellipsoid; they are directly measurable (provided the BABAR vertexing resolution is properly taken into account), and carry information about $\beta$ functions and emittances (Sec. 6). In the limiting case where the $e^{+}$and $e^{-}$beam sizes are pair-wise equal, one recovers the familiar expressions valid in single-ring colliders:

$\sigma_{i \mathcal{L}}=\sigma_{i b} / \sqrt{2}=\Sigma_{i} / 2$.

The specific luminosity $\mathcal{L}_{s p}$ is defined as the luminosity per bunch and per unit bunch currents. Integrating Eq. 6 yields $\mathcal{L}_{s p} \sim 1 / \Sigma_{x} \Sigma_{y}$ : this observable, which is continuously monitored using high-rate, radiative-Bhabha events, can thus be used to constrain certain $e^{ \pm}$beam-size combinations, as discussed in Sec. 9.

\subsection{The Hourglass Effect}

In the vicinity of the IP, the $e^{ \pm}$trajectories are straight lines, and the IP angular spread $\sigma_{i b}^{\prime}$ $(i=x, y)$ induces a longitudinal dependence of the transverse beam size:

$\sigma_{i b}^{2}(z)={\sigma_{i b}^{*}}^{2}+{\sigma_{i b}^{\prime}}^{2}\left(z-z_{i b}^{w}\right)^{2}$

where $z_{i b}^{w}$ is the longitudinal position of the optical waist, $\sigma_{i b}^{*}$ is the IP spot size, and

$\sigma_{i b}^{\prime}=\sqrt{\frac{\epsilon_{i b}}{\beta_{i b}^{*}}}$

is the RMS angular divergence. Equivalently,

$\sigma_{i b}^{2}(z)=\epsilon_{i b} \beta_{i b}^{*}\left(1+\frac{\left(z-z_{i b}^{w}\right)^{2}}{\beta_{i b}^{* 2}}\right)$.

This hourglass effect is noticeable only when $\beta_{i b}^{*}$ is smaller than or comparable to the bunch lengths. In $e^{ \pm}$rings this is typically true in the vertical only: $\sigma_{y b}(z)$ increases with the distance to the waist, while within a few bunch lengths of the IP, the horizontal beam sizes remain essentially constant (except possibly in the presence of strong beam-beam effects).

The dominantly Gaussian shape of the longitudinal luminosity distribution (Eq. 6) is modified, through its $\Sigma_{y}$ dependence, by an hourglass factor function of $\beta_{y+}^{*}$ and $\beta_{y-}^{*}$ (the $\beta_{x}^{*}$ dependence 
is negligible). This can be expressed in terms of a single effective IP $\beta$-function:

$$
\beta_{y, \text { eff }}^{*}=\sqrt{\frac{\epsilon_{y-} \beta_{y-}^{*}+\epsilon_{y+} \beta_{y+}^{*}}{\epsilon_{y-} / \beta_{y-}^{*}+\epsilon_{y+} / \beta_{y+}^{*}}}
$$

provided the two optical waists occur at the same location $\left(z_{y-}^{w}=z_{y+}^{w}\right)$. The problem simplifies if either the vertical $e^{ \pm}$emittances or IP $\beta$ functions are equal (both conditions are satisfied in singlering $e^{+} e^{-}$colliders). Simultaneous measurements of $\beta_{y, \text { eff }}^{*}$ and $\Sigma_{z}$ are presented in Sec. 5 .

The vertical luminous size $\sigma_{y} \mathcal{L}$ also increases as a function of the distance to the waist, at a rate that depends on $\beta_{y+,-}^{*}$ and $\epsilon_{y+,-}$, albeit with a slightly different functional dependence from that of $\beta_{y, \text { eff }}^{*}$ above. For such measurements the IP $\beta$ functions of the LER and HER are assumed to be identical $\left(\beta_{y+}^{*}=\beta_{y-}^{*}=\beta_{y}^{*}\right)$, as are the positions of the optical waists $\left(z_{y+}^{w}=z_{y-}^{w}\right)$. From the fit to the $z$-dependence of the vertical luminous size, one then extracts an effective vertical emittance:

$\epsilon_{y, \mathrm{eff}}=2 \frac{\epsilon_{y+} \epsilon_{y-}}{\epsilon_{y+}+\epsilon_{y-}}$

and a common value of $\beta_{y}^{*}$, as presented in Sec. 6 .

\subsection{The Boost Formalism}

The luminous products of $e^{+} e^{-}$collisions provide another experimentally measurable quantity. The boost vector $\mathbf{B}$ is defined as the total threemomentum of the $\mu^{+} \mu^{-}$pair. The distributions of the horizontal and vertical angles of this vector, or boost angles, and in particular the spatial dependence of their mean and RMS spread, carry extensive information about the IP parameters of both beams.

In the coordinate system of the incoming electron beam, the horizontal boost angle $x_{\mathrm{B}}^{\prime}$ of a muon pair is related to the angles $x_{-}^{\prime}$ and $x_{+}^{\prime}$ of the parent electron and positron by

$$
\begin{aligned}
x_{\mathrm{B}}^{\prime} & =\mathrm{B}_{x} /|\mathbf{B}| \\
& \approx\left(E_{-} x_{-}^{\prime}-E_{+} x_{+}^{\prime}\right) /\left(E_{-}-E_{+}\right) \\
& =f_{-} x_{-}^{\prime}-f_{+} x_{+}^{\prime},
\end{aligned}
$$

where the total momentum has been approximated by the difference in beam energies, and $f_{-(+)}$is the ratio of the electron (positron) beam energy to the difference of the two beam energies.
The mean horizontal boost angle is given by

${\overline{x^{\prime}}}_{\mathrm{B}}(z)=\frac{\int\left(f_{-} x_{-}^{\prime}-f_{+} x_{+}^{\prime}\right) \rho_{+} \rho_{-}}{\int \rho_{+} \rho_{-}}$,

where the integrals are performed over time and all transverse coordinates. When the two beams cross the IP with a non-zero mean angle $x_{\circ}^{\prime}$ and a half crossing-angle $\theta_{c}$, the mean horizontal boost angle depends on the emittances and $\beta$ functions of both beams:

$$
\begin{aligned}
\overline{x^{\prime}} \mathrm{B}= & x_{\circ}^{\prime}+\theta_{c}\left\{f_{-}+f_{+}-\right. \\
& \left.\frac{2 z^{2}}{\Sigma_{x}^{2}}\left(f_{-} \frac{\epsilon_{x-}}{\beta_{x-}^{*}}+f_{+} \frac{\epsilon_{x+}}{\beta_{x+}^{*}}\right)\right\} .
\end{aligned}
$$

The position of the horizontal luminous centroid depends on the relative horizontal beam sizes:

$\bar{x}_{\mathcal{L}}=z\left(x_{\circ}^{\prime}+\theta_{c} \frac{\sigma_{x+}^{2}-\sigma_{x-}^{2}}{\sigma_{x+}^{2}+\sigma_{x-}^{2}}\right)$,

and so does, therefore, the horizontal luminous tilt $\bar{x}_{\mathcal{L}}=\frac{\delta \bar{x}_{\mathcal{L}}}{\delta z}$. Combining the measured horizontal luminous tilt and mean boost angle, and assuming limits on the ratio of the horizontal IP spot sizes, provides a reliable estimate of the horizontal crossing angle:

$\overline{x^{\prime}}{ }_{\mathrm{B}}-\overline{x^{\prime}} \mathcal{L} \approx \theta_{c}\left(f_{-}+f_{+}-\frac{\sigma_{x+}^{2}-\sigma_{x-}^{2}}{\sigma_{x+}^{2}+\sigma_{x-}^{2}}\right)$

(using the very good approximation of $z \ll \beta_{x}^{*}$ ). Examples of such measurements are detailed in Sec. 7.3.2.

When the beams collide with small enough a crossing angle, the spread in horizontal boost angle is given by

${\sigma_{x_{\mathrm{B}}^{\prime}}}^{2} \approx \frac{f_{-}^{2} \epsilon_{x-}}{\beta_{x-}^{*}}+\frac{f_{+}^{2} \epsilon_{x+}}{\beta_{x+}^{*}}=f_{-}^{2}{\sigma_{x_{-}^{\prime}}^{2}}^{*}+f_{+}^{2} \sigma_{x_{+}^{\prime}}^{2}$

where we have used the fact that $\beta_{x}^{*}$ is considerably larger than the bunch lengths. Assuming similar horizontal angular divergences for the two beams, the horizontal-boost angular spread $\sigma_{x_{\mathrm{B}}^{\prime}}$ is largely determined by the angular divergence of the higher-energy $e^{-}$beam.

In the vertical plane, the boost angular spread is given by the more general expression of Eq. 14 . 


$$
\begin{aligned}
\sigma_{y_{\mathrm{B}}^{\prime}}^{2} & =\frac{\int\left(f_{-} y_{-}^{\prime}-f_{+} y_{+}^{\prime}\right)^{2} \rho_{+} \rho_{-}}{\int \rho_{+} \rho_{-}}-\left[\bar{y}_{\mathrm{B}}^{\prime}(z)\right]^{2} \\
& =\frac{\left\{\left(\epsilon_{y-} \beta_{y-}^{*}+\epsilon_{y+} \beta_{y+}^{*}\right)\left(f_{-}^{2} \frac{\epsilon_{y-}}{\beta_{y-}^{*}}+f_{+}^{2} \frac{\epsilon_{y+}}{\beta_{y+}^{*}}\right)+\frac{\epsilon_{y-}}{\beta_{y-}^{*}} \frac{\epsilon_{y+}}{\beta_{y+}^{*}}\left(f_{-} \alpha_{y+} \beta_{y+}^{*}-f_{+} \alpha_{y-} \beta_{y-}^{*}\right)^{2}\right\}}{\left[\epsilon_{y-} \beta_{y-}^{*}\left(1+\alpha_{y-}^{2}\right)+\epsilon_{y+} \beta_{y+}^{*}\left(1+\alpha_{y+}^{2}\right)\right]}
\end{aligned}
$$

Its $z$-dependence is determined by the $\alpha$ terms, and receives observable contributions from both beams. As a consequence, the boost angle depends on where the collision occurs with respect to the optical waists of the two beams. This correlation is most naturally expressed in the coordinate system defined by the axes of the luminous ellipsoid. Provided the $y-z$ crossing angle is zero, the mean vertical angle of those electrons that contribute to the luminosity at a given point $(x, y, z)$ is related to their vertical position $y$ by

$$
\begin{aligned}
{\overline{y^{\prime}}}_{-} & =\frac{\int y_{-}^{\prime} \rho_{+} \rho_{-}}{\int \rho_{+} \rho_{-}} \\
& =y \frac{z-z_{y-}^{w}}{{\beta_{y-}^{*}{ }^{2}+\left(z-z_{y-}^{w}\right)^{2}}^{2}} .
\end{aligned}
$$

The mean vertical boost angle can thus be written

$$
\begin{aligned}
{\overline{y^{\prime}}}_{\mathrm{B}}= & f_{-}{\overline{y^{\prime}}}_{-}-f_{+}{\overline{y^{\prime}}}_{+} \\
= & y \frac{f_{-}\left(z-z_{y-}^{w}\right)}{\beta_{y-}^{*}{ }^{2}+\left(z-z_{y-}^{w}\right)^{2}}- \\
& y \frac{f_{+}\left(z-z_{y+}^{w}\right)}{\beta_{y+}^{*}+\left(z-z_{y+}^{w}\right)^{2}} \\
\frac{\delta \bar{y}^{\prime} \mathrm{B}}{\delta y}(z)= & \frac{f_{-}\left(z-z_{y-}^{w}\right)}{\beta_{y-}^{*}{ }^{2}+\left(z-z_{y-}^{w}\right)^{2}} \\
& -\frac{f_{+}\left(z-z_{y+}^{w}\right)}{{\beta_{y+}^{*}}^{2}+\left(z-z_{y+}^{w}\right)^{2}} .
\end{aligned}
$$

This relation is altered by (partially correlated) measurement errors on $y_{\mathrm{B}}^{\prime}$ and on the vertex $y$ coordinate. Assuming Gaussian resolution functions, the observed $y-{\overline{y^{\prime}}}_{\mathrm{B}}$ correlation obeys

$$
\left(\frac{\delta{\overline{y^{\prime}}}_{\mathrm{B}}}{\delta y}\right)_{\mathrm{obs}}=\frac{\sigma_{y y_{\mathrm{res}}^{\prime}}}{\sigma_{y \mathcal{L}^{2}+\sigma_{y_{\mathrm{res}}}^{2}}}+
$$

$$
\begin{aligned}
& \frac{\sigma_{y \mathcal{L}}^{2}}{\sigma_{y \mathcal{L}^{2}}+\sigma_{y_{\mathrm{res}}}^{2}}\{ \\
& \frac{f_{-}\left(z-z_{y-}^{w}\right)}{\beta_{y-}^{*}{ }^{2}+\left(z-z_{y-}^{w}\right)^{2}}- \\
& \left.\frac{f_{+}\left(z-z_{y+}^{w}\right)}{\beta_{y+}^{*}{ }^{2}+\left(z-z_{y+}^{w}\right)^{2}}\right\}
\end{aligned}
$$

where the vertical vertexing resolution $\sigma_{y_{\text {res }}}$ and vertexing-trajectory correlation $\sigma_{y y_{\text {res }}^{\prime}}$ can be extracted from the data.

These relationships illustrate the dependence of the boost angles on the LER and HER emittances, IP $\beta$ functions and waist positions. Precise measurement of the means and spreads of these angles, including their spatial dependence, allows the extraction of the individual beam parameters as discussed in Sec. 7 .

\subsection{Lattice diagnostics}

Lattice functions are measured one ring at a time, by exciting the beam horizontally or vertically at the corresponding betatron frequency, and by recording, on a thousand consecutive revolutions, synchronized readings of all the beam position monitors (BPMs). Such turn-by-turn orbits are recorded both at the nominal beam energy, and with the RF frequency varied up and down by typically $0.5 \mathrm{kHz}$ to determine in addition the dispersion function. Harmonic analysis of these data provides high-precision measurements of the phase and amplitude, at each BPM, of the coherent component of the transverse motion of the beam. The procedure is always carried out in single-bunch mode to eliminate any risk of multibunch-induced distortions of the betatron motion. The data can be analyzed either in terms of uncoupled betatron functions, or exploited to infer a full model of the ring optics with 
minimal a priori assumptions.

The first approach is that of the phase-advance method [13], which is well established and relatively straightforward to implement. Briefly, relative phase advances between three consecutive BPMs allow to determine the ratio of the $\beta$-function measured at each of these BPMs, to that predicted, at the same location, by the optical model of the lattice. Repeating the procedure around the ring yields, at any location, the values of $\beta$ and $\alpha-$ or more precisely the ratio between their actual and predicted values. In practice however, the accuracy of the measurement is degraded, in the vicinity of the IP, by unfavorable phase relationships between consecutive BPMs. In addition, the formalism implemented for PEP-II is fundamentally uncoupled, and its applicability questionable in the presence of strong local $x-y$ coupling, as is for instance the case in the interaction region of the LER.

In contrast, the "model-independent analysis" (MIA) approach [14] is based on a fully-coupled formalism, and designed to remain insensitive to modeling assumptions. Starting from the resonantly excited turn-by-turn BPM data, it reconstructs a detailed model of the lattice that includes the fitted strengths of all the normal and skew quadrupoles (or quadrupole families), as well as quadrupole feed-down from offset sextupoles. The final result is an optics model of the lattice, called a virtual machine [15], that in principle mirrors the optical properties of the actual ring. While the method often proved very powerful, the results are sometimes affected by the varying quality of the BPM data, and their interpretation may be delicate because of the intricacy of the fitting procedure.

\subsection{Use of synchrotron-light profile moni- tors}

By combining, in each ring, profile-monitor data with measured lattice functions, one can in principle extract the eigenemittances and predict the $e^{ \pm}$spot sizes at the IP.

PEP-II is equipped with several beam-profile monitors. These devices determine locally the $x$ and $y e^{ \pm}$beam sizes, and in most cases also the associated $x-y$ tilt. In the LER, a visi- ble synchrotron-light monitor (SLML) [16], located in a high-coupling region $30 \mathrm{~m}$ from the $\mathrm{IP}$, is complemented by a pin-hole X-ray camera (SXML) [17] at a separate, more favorable location. The HER is equipped with one visiblelight monitor (SLMH) in a nominally uncoupled region [18]. A synchrotron-light interferometer, associated with each SLM, splits off a portion of the light from the same source to provide a higher-resolution measurement of the narrow vertical beam size [19-21].

In the presence of $x-y$ coupling, the simplified phase-space description of Eq. 1 can be generalized using the one-turn matrix formalism developed in Refs. $[12,22]$. In this context, the projected beam sizes $\left(\sigma_{x}, \sigma_{y}\right)$ anywhere around a ring can be expressed in terms of the emittances of the two transverse normal modes and of ten lattice parameters, that together fully describe the beam phase space at the location considered:

$$
\begin{aligned}
\sigma_{x}^{2}= & \beta_{1} \epsilon_{1} g^{2}+ \\
& \left(\beta_{2} w_{22}^{2}+2 \alpha_{2} w_{22} w_{12}+\gamma_{2} w_{12}^{2}\right) \epsilon_{2}+\sigma_{\eta_{x}}^{2} \\
\sigma_{y}^{2}= & \beta_{2} \epsilon_{2} g^{2}+ \\
& \left(\beta_{1} w_{11}^{2}-2 \alpha_{1} w_{11} w_{12}+\gamma_{1} w_{12}^{2}\right) \epsilon_{1}+\sigma_{\eta_{y}}^{2} .
\end{aligned}
$$

Here, $w$ is a $2 \times 2$ quasi-symplectic matrix describing the coupling between $x$ and $y, g^{2}=1-\operatorname{det}(w)$, $\epsilon_{i}(i=1,2)$ is the emittance of eigenmode $i, \beta_{i}$ is the corresponding $\beta$ function, $\alpha_{i}$ and $\gamma_{i}$ are defined by Eqs. 2 and $3, \eta_{x, y}$ is the horizontal or vertical dispersion, and $\sigma_{\eta_{x, y}}=\eta_{x, y} \Delta p / p$ represents the dispersive contribution to the beam size.

Lattice properties are measured by resonant excitation as described in Sec. 3.6. The BPM data are analyzed by the MIA method [15], and fitted in the context of the LEGO package [23,24] to produce a set of fully-coupled lattice functions. As a byproduct, this procedure also predicts, for later comparison, the $e^{ \pm}$eigenemittances and the IP spot sizes in the absence of beam-beam interactions.

The eigenemittances, being invariants, should not depend on where in the ring they are measured. They can therefore be inferred, using Eqs. 17 and 18, from the lattice functions at the source point of the profile monitor, combined with the transverse beam sizes measured at the same 
location.

In addition, because $x-y$ coupling may also cause a tilted profile-monitor image, it is possible to further constrain the analysis by a third equation that involves the tilt angle $\psi$ measured at the monitor:

$\tan (2 \psi)=2 \sigma_{x y} /\left(\sigma_{x}^{2}-\sigma_{y}^{2}\right)$

where

$\sigma_{x y}=g\left[\left(\beta_{2} w_{22}+\alpha_{2} w_{12}\right) \epsilon_{2}-\left(\beta_{1} w_{11}\right.\right.$ $\left.\left.-\alpha_{1} w_{12}\right) \epsilon_{1}\right]+\sigma_{\eta_{x}} \sigma_{\eta_{y}}$.

An SVD fit making use of all the available measurements improves the determination of the emittances. This approach is especially important for the LER, which is heavily coupled at the SLML, but where the SXML monitor provides a redundant set of equations.

Once the eigenemittances have been determined from profile-monitor data, they can be combined with the fitted lattice functions at the IP to predict the $e^{ \pm}$transverse beam sizes at the collision point: these studies are presented in detail in Sec. 8.

\section{Online Monitoring of the Luminous Re- gion}

The quasi-elastic scattering processes $e^{+} e^{-} \rightarrow$ $\mu^{+} \mu^{-}, e^{+} e^{-}$are chosen for our purposes, because of their simple, background-free topology and low track multiplicity, which translate into faster event processing (Sec. 4.1). The three-dimensional distribution of event vertices (Sec. 4.2) monitors the position, orientation, size and shape of the luminous ellipsoid. The convoluted bunch length $\Sigma_{z}$, effective vertical $\beta$ function $\beta_{y \text {,eff }}^{*}$ and waist position $z_{y}^{w}$ are also continuously monitored, using algorithms developed for the offline analysis of the longitudinal luminosity distribution (Sec. 5). The boost vector of reconstructed $\mu^{+} \mu^{-}$pairs is used online to track the horizontal $e^{+} e^{-}$crossing angle and the energyweighted angular divergence; the presentation of the latter two analyses is deferred until Sec. 7 .

\subsection{Data Acquisition and Online Process- ing}

When PEP-II operates at a luminosity of $10^{34} \mathrm{~cm}^{-2} s^{-1}$, about $1500 e^{+} e^{-} \rightarrow e^{+} e^{-}, \mu^{+} \mu^{-}$ events are recorded per minute in $B A B A R$. Event data are collected on a farm of 32 Linux nodes. Based on the Level-1 trigger flags, subsets of events are redirected to eight monitoring CPUs that run the full BABAR tracking software. Charged-particle trajectories are reconstructed using hits recorded in the SVT and DCH, and the resulting track list is fed to a selection algorithm that retains the appropriate two-prong events.

Events accepted for online monitoring are required to have exactly two oppositely-charged, well-reconstructed tracks. Track quality criteria include a minimum of 5 hits in the SVT and between 20 and 50 hits in the DCH; the track polar angle $\theta$ must satisfy $\cos \theta<0.9$ to reject trajectories traversing the permanent-magnet beam-separation dipoles. Two-body final-state kinematics are enforced by requiring an invariant mass above $9.5 \mathrm{GeV}$, with the two tracks almost back-to-back in the transverse plane $(\cos \Delta \phi<$ -0.99 , where $\Delta \phi$ is the difference in azimuthal angles). Exploiting the asymmetry of beam energies, cosmic rays are completely removed by requiring that the two tracks satisfy $1 / \tan \theta_{1}+$ $1 / \tan \theta_{2}>0.5$. The $\chi^{2}$ per degree of freedom of the vertex fit must be less than 3 . In order to fully exploit the SVT resolution, the boost analysis is further restricted to muon pairs, rejecting Bhabha events by requiring that the total EMC energy be less than $3.0 \mathrm{GeV}$. The offline analyses discussed in Secs. 5 to 7 apply the same primary event selection as that described above, with somewhat tightened criteria for some applications; such cases are mentioned in the corresponding sections.

\subsection{Online Characterization of the Lumi- nous Ellipsoid}

The spatial coordinates of the retained event vertices are continuously histogrammed. All distributions are available for viewing in real time over network links by many clients; they are typically refreshed once per second (faster update rates are possible). The mean and standard devi- 
ation of these distributions, which reflect the centroid location and the RMS size of the luminous region, are regularly updated and, after correction for experimental resolution, shipped to the accelerator control system. Here the typical refresh period is 10 minutes, which at a luminosity of $10^{34} \mathrm{~cm}^{-2} \mathrm{~s}^{-1}$ corresponds to 7000 accumulated events (there is insufficient CPU available to process online all the two-prong events that $B A B A R$ records). All measurements, together with other accelerator parameters, are also archived for long-term monitoring and offline study.

\subsubsection{Luminous Centroids and Beam IP Angles.}

The knowledge and long-term stability of the interaction-region (IR) orbit is crucial to ensure the reproducibility of the ring optics. The expected resolution on the position of the luminous centroid is about $1 \mu \mathrm{m}, 0.5 \mu \mathrm{m}$, and $0.1 \mathrm{~mm}$ for $\bar{x}_{\mathcal{L}}, \bar{y}_{\mathcal{L}}$ and $\bar{z}_{\mathcal{L}}$ respectively, as confirmed by the sample-to-sample scatter in the time history of the actual centroids (Fig. 3).

In $e^{+} e^{-}$colliders, the transverse IP position is typically monitored using BPMs located one or more quadrupoles away from the IP. Such a measurement is compared to the online BABAR data in Fig. 3 . The IP-position variations reported by the BPMs are inconsistent in amplitude, and a couple of hours off in phase, compared to those reported by BABAR. This is attributed to the combination of several factors: lattice-modeling errors (which affect the transfer matrix from the BPMs to the IP); drifts in the BPM electronics; and mechanical motion (thermal expansion of mechanical supports, differential long-term ground motion), that distorts, in a time-dependent manner, the relation between the beam positions and angles at the IP and those at the BPMs. In contrast, the $B A B A R$ IP position directly measures the quantity of interest, on an absolute scale set by the extremely precise and stable mechanical structure of the SVT. It has proven a valuable tool to diagnose large thermally-induced motion in the IR, or to properly restore the horizontal IP position (to which the $e^{+}$optics is rather sensitive) after long interruptions.

Similar observations apply to the vertical

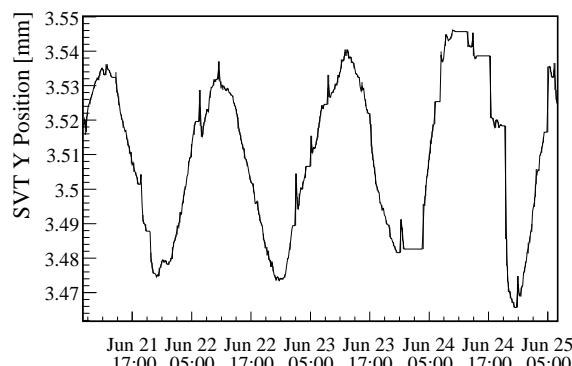

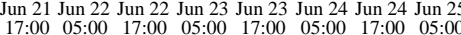

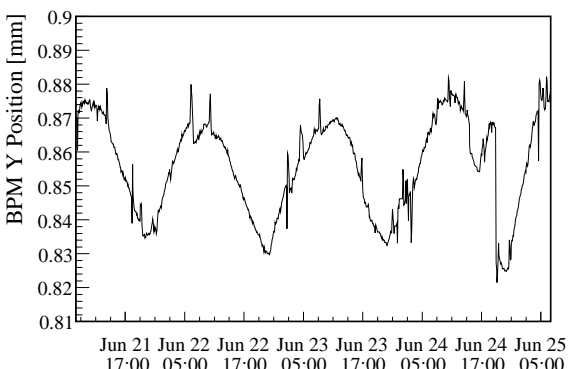

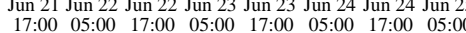

Figure 3. Time history of the vertical IP position. Top: vertical luminous centroid reported by BABAR. Bottom: y position of the $e^{+}$beam at the IP, measured by the BPMs. The BPM data have an arbitrary zero offset. The sine-like behavior is caused by day-night temperature variations.

(Fig. 4, top) and horizontal luminous tilts, for which BABAR measurements are more reliable than the individual beam angles extracted from BPM data. In the vertical plane, the ribbon beams are held in tight relative alignment by an accelerator feedback loop that maximizes the instantaneous luminosity; the actual value of the common vertical IP angle is narrowly constrained by synchrotron-radiation heating of IR beam pipes, and needs to be closely monitored. In the horizontal plane, the difference between the mean boost angle and luminous tilt yields the horizontal crossing angle (Eq. 12), as detailed in Sec. 7 .

The longitudinal position $z_{c} \sim \bar{z}_{\mathcal{L}}$ of the collision point, which ideally should coincide with 


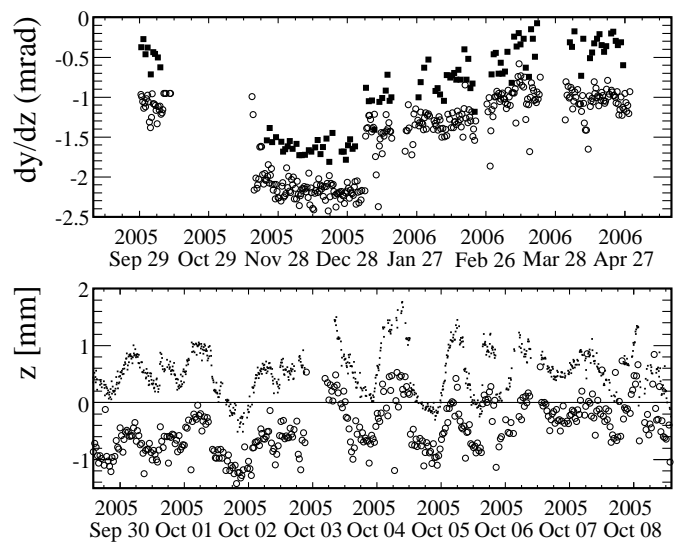

Figure 4. Top: history of the vertical luminous tilt $\overline{y^{\prime}} \mathcal{L}$ (full squares), and vertical $e^{-}$IP angle reported by the beam position monitor system (open circles). The steps reflect deliberate adjustments of the orbit after major accelerator maintenance. Bottom: history of the longitudinal luminous centroid (dots), and half of the differential $\mathrm{RF}$ phase between the two rings (open circles). The RF-phase data have an arbitrary zero offset.

that of the optical waists, is not directly measurable by accelerator techniques: only its relative variation averaged over the bunch train can be inferred from empirical, luminosity-driven adjustments of the RF-phase difference between the two rings. The SVT resolution is sufficient to routinely detect sub-mm changes in the trainaveraged longitudinal luminous centroid $\left\langle\bar{z}_{\mathcal{L}}\right\rangle$ (Fig. 4, bottom). The technique can be refined to measure the variation of $z_{c}$ along the bunch train (Sec. 5.3.1), revealing systematic variations in longitudinal centroid location that depend on the bunch pattern and total beam current.

\subsubsection{Luminous size, bunch length and vertical $\beta$ function.}

The transverse projections of the event-vertex distribution provide a direct measure of the horizontal luminous size $\sigma_{x \mathcal{L}}$. Because the actual vertical luminous size $\left(\sigma_{y \mathcal{L}} \sim 3 \mu \mathrm{m}\right)$ is much smaller than the vertexing resolution $\left(\sigma_{y_{\mathrm{res}}} \sim 25-35 \mu \mathrm{m}\right.$ depending on the cuts), the apparent vertical luminous size directly measures that resolution, and must be taken into account when reporting the horizontal size. An early application of luminoussize monitoring is presented in Sec. 7.2.2: when the horizontal tunes were brought close to $\nu_{x}^{ \pm}=$ 0.5 in 2003, the combination of an actual reduction in $\beta_{x}^{*-}$, and of the dynamic- $\beta$ effect in both rings, caused $\sigma_{x \mathcal{L}}$ to shrink from about 90 to $67 \mu \mathrm{m}$.

As the resolution of the vertexing algorithms is too coarse to determine the actual value of $\sigma_{y \mathcal{L}}$ in real time, a more precise method was developed that exploits the azimuthal dependence of the transverse impact parameters of the two finalstate muons. This offline approach allows one to calibrate the experimental resolution using the data themselves, and to measure the hourglass effect through the $z$-dependence of the vertical luminous size (Sec. 6).

At the end of each data-taking run (approximately 1 hour), the longitudinal luminosity distribution is used to monitor $\Sigma_{z}, \beta_{y \text {,eff }}^{*}, z_{c}$, and $z_{y}^{w}$. The full distribution and the corresponding fit results are archived for later analysis. These studies are detailed in Sec. 5.1 below.

\section{Longitudinal Luminosity Distribution}

\subsection{Measurement of Hourglass Parame- ters}

\subsubsection{Principle of the Method}

When fitting Eq. 6 to the $z$-distribution of $\mu^{+} \mu^{-}$event vertices (Fig. 5), the convoluted bunch length $\Sigma_{z}$ and collision point $z_{c}$ are determined by the Gaussian component of the distribution, while the effective vertical IP $\beta$-function $\beta_{y \text {,eff }}^{*}$ (Eq. 10) modifies the width of the Gaussian through the $z$-dependence of the convoluted beam size $\Sigma_{y}$. In addition, if the optical waist $z_{y}^{w}$ of either beam does not overlap with the collision point, it introduces a detectable left-right asymmetry in the distribution.

Because the length of the luminous region $\left(\sigma_{z \mathcal{L}} \sim 7.5 \mathrm{~mm}\right)$ is much larger than the corresponding vertexing resolution $\left(\sigma_{z_{\text {res }}} \sim 25 \mu \mathrm{m}\right)$, measurements extracted from the longitudinal luminosity profile are insensitive to the details of 


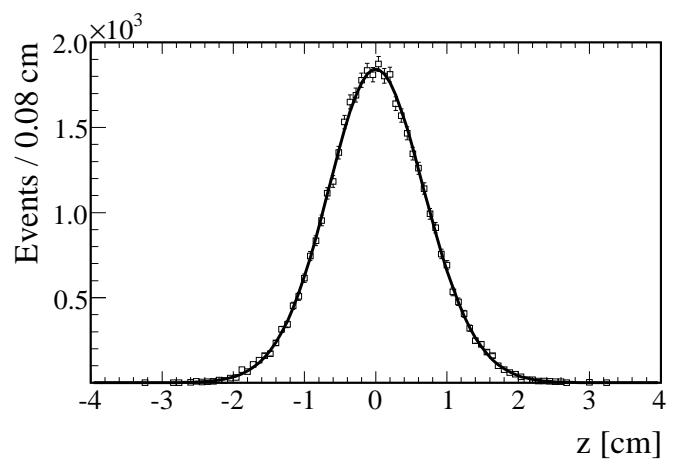

Figure 5. Measured longitudinal luminosity distribution $\frac{d \mathcal{L}}{d z}$, after all cuts (circles). The curve is the result of the fit.

the resolution function (in contrast, the vertical luminous-size measurements of Sec. 6 rely on an exacting control of the vertical vertexing resolution).

\subsubsection{Potential Biases and Systematic Un- certainties}

Any effect that distorts the shape of the measured $\frac{d \mathcal{L}}{d z}$ distribution can potentially bias the measurements of $\Sigma_{z}$ and $\beta_{y \text {,eff }}^{*}$. In particular, the event reconstruction efficiency (Fig. 6, top) must be independent of $z$ throughout the luminous region. Small distortions induced by geometricalacceptance effects were investigated using real data samples as well as a full GEANT 4 simulation [25] that incorporates a complete description of the BABAR response. The event selection criteria were tightened to eliminate pooracceptance regions and minimize residual resolution and background effects. An example is shown in Fig. 6 (bottom): if left undetected, a small localized gap in SVT coverage would have caused a $z$-dependent distortion of the distribution, inducing a $\beta_{y, \text { eff }}^{*}$ bias of up to $0.5 \mathrm{~mm}$.

The fitting procedure was validated using parameterized simulations of $\mu^{+} \mu^{-}$events. Highstatistics $\frac{d \mathcal{L}}{d z}$ distributions were generated under the assumptions of Sec. 3.1, covering a wide range
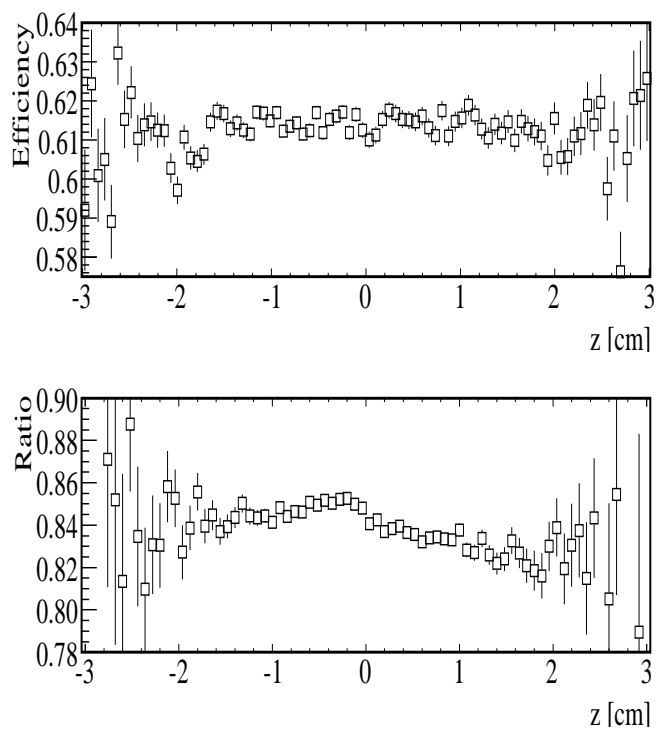

Figure 6. Top: $z$-dependence of the $\mu$-pair reconstruction efficiency, after all event-selection cuts, determined using a full Monte Carlo simulation of the BABAR detector. Bottom: ratio of the measured longitudinal luminosity profile after all cuts, to that obtained without removing the region with degraded SVT tracking efficiency.

of assumed emittances $\epsilon_{y \pm}$, IP $\beta$-functions $\beta_{y \pm}^{*}$ and bunch lengths $\sigma_{z \pm}$. The resulting fitted values of $\Sigma_{z}$ and $\beta_{y, \text { eff }}^{*}$ are, as expected, strongly anti-correlated; they agree with the true (generated) values within $2 \%$ provided the fitted distribution contains at least 25,000 events.

When the $e^{+}$and $e^{-}$vertical waists coincide, the fitted values of $\Sigma_{z}$ and $\beta_{y \text {,eff }}^{*}$ are insensitive to the distance between the common waist and the collision point. But when the two waists occur at different locations, the longitudinal luminosity distribution flattens slightly. The potential quantitative impact is illustrated in Fig. 7: a $2 \mathrm{~cm}$ separation between vertical waists biases $\Sigma_{z}$ by $0.1 \mathrm{~mm}$ at most, but $\beta_{y \text {,eff }}^{*}$ by almost $4 \mathrm{~mm}$. In practice, the longitudinal positions of the collision point and of the vertical waists are unknown: they are empirically adjusted by the PEP-II op- 
erators to optimize the luminosity, to an accuracy estimated to $\sim 0.5 \mathrm{~cm}$. The resulting systematic uncertainty on $\beta_{y, \text { eff }}^{*}$ is less than $0.5 \mathrm{~mm}$, and that on $\Sigma_{z}, z_{c}$ and $z_{y}^{w}$ negligible.
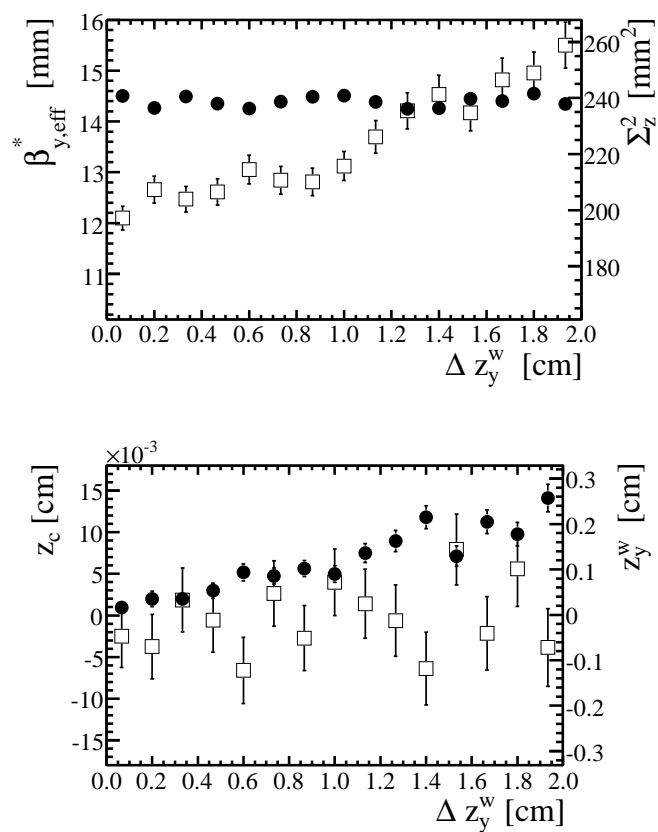

Figure 7. Top: influence of the true waist separation $\Delta z_{y}^{w}=\left|z_{y+}^{w}-z_{y-}^{w}\right|$, in simulated $\frac{d \mathcal{L}}{d z}$ distributions, on the fitted values of $\beta_{y \text {,eff }}^{*}$ (open squares, left scale) and $\Sigma_{z}^{2}$ (full circles, right scale). Bottom: same, but for the fitted collision point $z_{c}$ (open squares) and assumed common waist location $z_{y}^{w}$ (full circles). The true (generated) collision point remains fixed at $z_{c}=0$.

Other beam-dynamics effects can also impact the shape of the longitudinal luminosity profile. Because the abort gap (a few tens of empty buckets at the end of the bunch train) induces a different RF-phase transient in the two rings, the longitudinal position $z_{c}$ of the collision point varies by up to $6 \mathrm{~mm}$ along the train (Sec. 5.3.1). This is taken into account, when accumulating the longitudinal luminosity distribution, by correcting the measured longitudinal vertex position of each event by a bucket-dependent $z$-offset determined directly from the data for each run $^{3}$. Slow drifts in the train-averaged longitudinal luminous centroid are similarly corrected.

The bunch lengths depend on the $e^{ \pm}$beam currents and RF voltages. These quantities are continuously recorded at six-minute intervals, and the luminous-region data appropriately segregated in batches corresponding to reasonably constant conditions. The RF voltage also varies within the train itself. This induces bunchlength modulation, which is directly measurable (Sec. 5.3.1), but sufficiently small not to affect train-averaged measurements in a significant fashion.

In the LER, the high bunch current coupled with RF-impedance effects induces a longitudinal asymmetry of 6 to $8 \%$ in the longitudinal $e^{+}$distribution [26]. The corresponding bias on $\beta_{y, \text { eff }}^{*}$ is small compared to other systematic uncertainties: the distortion of a simulated luminosity profile by a $10 \%$ asymmetry in the longitudinal positron distribution, shifts the fitted value of $\beta_{y \text {,eff }}^{*}$ by less than $0.5 \mathrm{~mm}$.

Another potential distortion of the longitudinal vertex distribution is associated with the IP luminosity feedback. In order to maintain headon collisions, the transverse position and vertical angle of the electron beam are "dithered" at a frequency of a few $\mathrm{Hz}$ and continuously adjusted to maximize the luminosity. The typical dither amplitude is $\sim 5-10 \%$ of the corresponding beam size, inducing a small $(\sim 1 \%)$ luminosity degradation that is largest at the waist: the net effect is a very slight flattening of the longitudinal luminosity profile. Simulating this procedure and fitting the resulting luminosity profile shows that the resulting bias on $\beta_{y \text {,eff }}^{*}$ remains below $0.1 \mathrm{~mm}$.

\footnotetext{
$\overline{{ }^{3} \text { Such corrections are applied in the offline analyses pre- }}$ sented in this section, but are neglected for the online diagnostics of Sec. 4.
} 


\subsubsection{History of Hourglass Parameters Measured at the PEP-II IP}

Figures 8 and 9 display the long-term evolution of the hourglass parameters extracted from the longitudinal luminosity distribution, under the assumptions listed in Sec. 3.1. The results are highly insensitive to variations in the event selection cuts. The instrumental systematic uncertainties are estimated to $\pm 1 \mathrm{~mm}$ on $\beta_{y \text {,eff }}^{*}$, and $\pm 0.5 \mathrm{~mm}$ on $\Sigma_{z}$. The waist $z_{y}^{w}$ shown in Fig. 9 is often slightly different from the collision point $z_{c}$. This feature is discussed in Sec. 9.

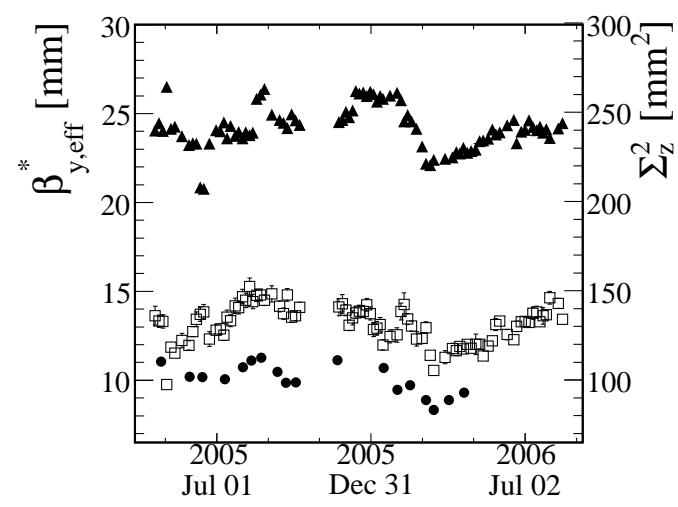

Figure 8. Right scale: one-year history of the squared convoluted bunch length $\Sigma_{z}^{2}$ (full triangles), as extracted from the fit to the longitudinalluminosity profile. Left scale: history of $\beta_{y \text {,eff }}^{*}$ (open squares), from the same fit as $\Sigma_{z}$. The full circles represent phase-advance measurements of $\beta_{y+,-}^{*}$, appropriately combined assuming $\epsilon_{y+}=$ $1.5 \mathrm{~nm}-\mathrm{rad}, \epsilon_{y-}=2.5 \mathrm{~nm}-\mathrm{rad}$ and neglecting $x-y$ coupling.

To investigate the potential impact of beambeam-induced distortions of the luminous region, the fit is also applied to luminosity distributions produced by the beam-beam simulations of Ref. [27], both at nominal and at very low bunch currents (where distortions should be negligible). At the lowest bunch currents, all fitted parame-

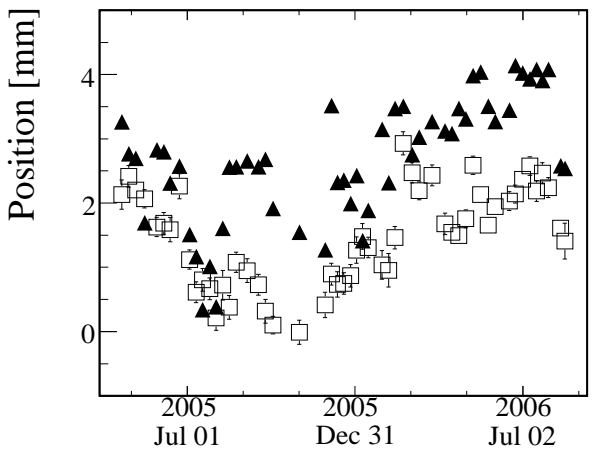

Figure 9. One-year history of the fitted longitudinal position of the collision point $z_{c}$ (black triangles) and of the (assumed common) vertical waist position $z_{y}^{w}$ (open squares), as extracted from the fit to the longitudinal-luminosity profile.

ters agree very well with the generated values; in particular, the "true" values of $\beta_{y \text {,eff }}^{*}$ and $\Sigma_{z}$ are reproduced to within $\sim 0.2$ and $\sim 0.1 \mathrm{~mm}$ respectively. The same applies at high bunch currents, except for $\beta_{y}^{*}$ which decreases by approximately $0.5 \mathrm{~mm}$. The same small dynamic- $\beta$ effect is apparent when comparing directly, at low and high bunch currents, the RMS widths of the vertical position and angle distribution of the $e^{ \pm}$macroparticles at the IP.

Also shown on Fig. 8 is a comparison with a set of uncoupled lattice functions measured in single-bunch mode by the phase-advance method (Sec. 3.6). The disagreement cannot be accounted for by systematic biases associated with the geometric acceptance of the detector, the event selection or reconstruction efficiencies or other instrumental effects. It cannot not be explained either by any of the beam-dynamics effects considered above; it persists under reasonable variations of the relative $e^{ \pm}$waist position, or of the vertical emittances assumed when combining the electron and positron lattice functions (Eq. 10). Similar or larger discrepancies are observed when comparing the same lattice-function measurements to IP $\beta$-functions determined using the boost method 
(Sec. 7). A possible explanation of these apparent inconsistencies is explored in Sec. 9.

\subsection{Bunch Length Measurements}

The sensitivity of the luminous length to moderate changes in RF voltage is illustrated in Fig. 10. This suggests to measure the individual $e^{+}$and $e^{-}$bunch lengths by analyzing the longitudinal shape of the luminosity distribution in the presence of known variations in RF voltage(s) and/or beam current(s), one beam at a time.

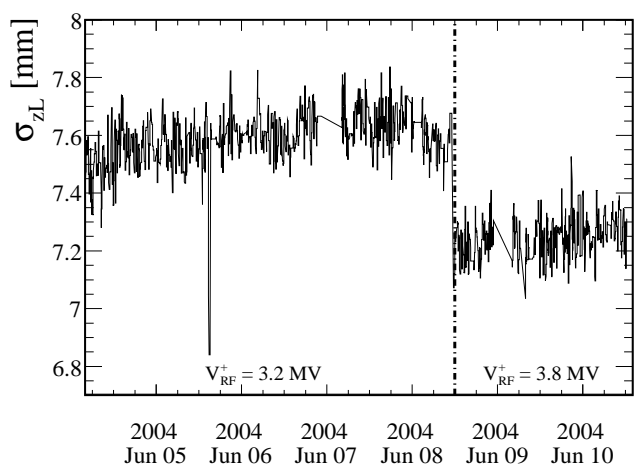

Figure 10. History of the RMS luminous length $\sigma_{z \mathcal{L}}$, before and after the LER RF voltage $V_{R F}^{+}$ was increased (dashed vertical line). The HER RF voltage $V_{R F}^{-}$remained constant.

We first analyzed large samples $(\sim 0.5-2.5 \times$ $\left.10^{6}\right)$ of $\mu^{+} \mu^{-}$events recorded at two different settings of the LER RF voltage, with $V_{R F}^{-}$fixed. The known $V_{R F}$-dependence of the bunch length

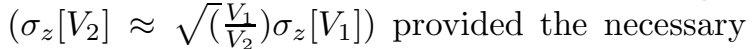
extra constraint. The results are summarized in Table 2. The fitted value of $\beta_{y \text {,eff }}^{*}$ in these particular data samples, is typical of the results shown in Fig. 8. If instead $\beta_{y, \text { eff }}^{*}$ is fixed to the significantly smaller value inferred from singlebunch lattice measurements during the same period, the $\chi^{2}$ of the fit degrades significantly, suggesting the results are less reliable; even then, both the $e^{-}$and $e^{+}$bunch lengths increase only slightly. These results are highly insensitive to variations in the portion of the bunch train included in the fit, or to the assumed values of parameters such as waist positions, emittances or horizontal IP $\beta$-functions in the two rings. In both rings, the longitudinal luminosity distribution yields bunch length measurements that, within statistical errors, are consistent with recent results (Table 3) using the more traditional streak-camera technique [26]. Earlier measurements that exploit the high-frequency roll-off of the bunch spectrum $[28,29]$ are also listed for comparison.

Table 2

Bunch length measurements extracted from the longitudinal luminosity distribution, varying $V_{R F}^{+}$. The results are quoted at the nominal bunch currents $I_{+}\left(I_{-}\right) \approx 1.4(0.9) \mathrm{mA}$, and at $V_{R F}^{+}\left(V_{R F}^{-}\right)=3.8(16) \mathrm{MV}$. The errors are statistical only. $\sigma_{z}$ and $\beta_{y}^{*}$ are in $\mathrm{mm}$.

\begin{tabular}{|c|cc|}
\hline Parameter & LER $\left(e^{+}\right)$ & HER $\left(e^{-}\right)$ \\
\hline$\beta_{y, \text { eff }}^{*}($ fitted $)$ & \multicolumn{2}{|c|}{$16.1 \pm 0.2$} \\
$\sigma_{z}($ fitted $)$ & $11.1 \pm 1.1$ & $11.1 \pm 1.1$ \\
$\chi^{2} /$ D.O.F. & \multicolumn{2}{|c|}{3.5} \\
\hline$\beta_{y}^{*}$ (phase advance) & 10.6 & 10.3 \\
$\sigma_{z}($ fitted $)$ & $11.5 \pm 0.4$ & $12.0 \pm 0.4$ \\
$\chi^{2} /$ D.O.F. & \multicolumn{2}{|c|}{30} \\
\hline
\end{tabular}

Table 3

Bunch length measurements by conventional accelerator techniques. The results are quoted at $I_{+}\left(I_{-}\right)=1.4(0.9) \mathrm{mA} /$ bunch, and at $V_{R F}^{+}$ $\left(V_{R F}^{-}\right)=3.8$ (16) MV. $\sigma_{z}$ is in $\mathrm{mm}$.

\begin{tabular}{|c|cc|}
\hline Parameter & LER $\left(e^{+}\right)$ & HER $\left(e^{-}\right)$ \\
\hline$\sigma_{z}$ (streak camera) & $11.8[26]$ & $10.7[26]$ \\
$\sigma_{z}$ (spectral method) & $12.4[29]$ & $11.6[28]$ \\
\hline
\end{tabular}

In a second experiment, we took advantage of a long coast-down during which the $e^{+}\left(e^{-}\right)$cur- 
rent decayed from 2.4 (1.5) A to 0.65 (1.0) A. The luminous length exhibits a clear beam-current dependence (Fig. 11). As streak-camera measurements suggest a strong (weak) dependence of the $e^{+}\left(e^{-}\right)$bunch length on the corresponding total current, the data was fitted under the assumption that $\sigma_{z+}$ varied linearly with current, and that $\sigma_{z-}$ remained fixed to the value determined in the first experiment. The fit yields $\sigma_{z+}=11.2 \pm$ $0.9 \mathrm{~mm}$ at full current $\left(I_{+}=1.5 \mathrm{~mA} /\right.$ bunch $)$, and $d \sigma_{z+} / d I_{+}=0.84 \pm 0.37 \mathrm{~mm} /(\mathrm{mA} /$ bunch $)$, fully consistent with, but less precise than, the corresponding streak-camera measurements [26].

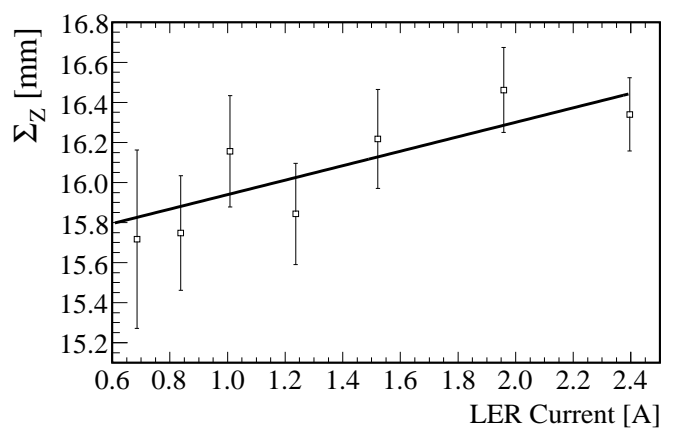

Figure 11. $e^{+}$-current dependence of the fitted value of $\Sigma_{z}$ measured during a long coast-down. The line shows the fitted current-dependence (see text). The HER bunch length and the IP $\beta$ functions are fixed in the fit.

Fig. 12 displays archived online measurements of $\Sigma_{z}$ at different accelerating voltages, and compares them to the quadratic sum of the $e^{+}$and $e^{-}$bunch lengths measured separately, at distant time intervals, by fitting the frequency spectrum at pickup electrodes in the HER and LER [28]. The two methods report comparable changes in luminous length, with a magnitude roughly consistent with the variations in accelerating voltage; the absolute length scales of the two sets of measurements agree within about $3 \%$. However, the more frequent luminous-region measurements exhibit systematic variations of up to $\pm 0.2 \mathrm{~mm}$, at apparently constant $\mathrm{RF}$ voltage, that remain to be understood. If these variations are taken as a measure of the systematic uncertainty on $\Sigma_{z}$, they translate into systematic uncertainties of roughly $\pm 2 \mathrm{~mm}$ on the values of $\sigma_{z+}$ and $\sigma_{z-}$ determined using RF-voltage changes. These uncertainties on the $e^{+}$and $e^{-}$bunch lengths are totally anti-correlated. The reason they are much larger, in relative terms, than that affecting $\Sigma_{z}$, is that the determination of the individual bunch lengths relies on the small difference between two $\Sigma_{z}$ values less than $1 \mathrm{~mm}$ apart.

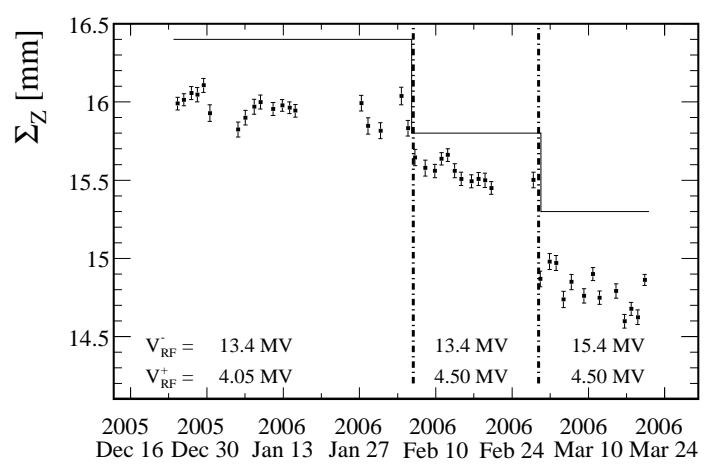

Figure 12. $\Sigma_{z}$ measurements based on the longitudinal luminosity profile (points with statistical error bars), and computed from bunch length measurements in the two rings by the spectral method (solid lines) at various gap voltages.

\subsection{Variation of Beam Parameters along the Bunch Train}

Accelerator fill patterns change on a time scale of days to months. Where relevant in this paper, care has been taken to analyze train-averaged data with consistent fill patterns and beam currents. But it is actually possible to study IP parameters as a function of the bucket number within the train. The $59.5 \mathrm{MHz}$ clock signal for 
the $B A B A R$ data-acquisition and trigger systems is synchronized with every 8th tick of the $476 \mathrm{MHz}$ PEP-II RF clock. This allows one to use the time stamp of the trigger, relative to the accelerator turn signal, to determine the approximate bucket number during which the final-state lepton pair was produced. More precise drift-chamber timing information identifies the exact bucket to within $\sim 2 \mathrm{~ns}$, or about half of the shortest bunch-tobunch separation.

\subsubsection{Gap transient and Bunch-length Modulation}

The variation, along the bunch train, of $\Sigma_{z}^{2}$ and $z_{c}$, are compared in Fig. 13 to an analytical prediction [30] of $\Sigma_{z}^{R F}$ (computed from the individual $e^{+}$and $e^{-}$bunch-length predictions) and of the differential RF-phase transient. This prediction takes into account the actual bunch pattern, bunch currents and RF complement to compute, in each ring separately, the expected RF loading, phase transient, RF-voltage and bunchlength variation along the train. The predicted amplitude and phase of the bunch-length modulation and phase transient are in satisfactory agreement with our measurement. Because the $\mathrm{RF}$ model yields only the zero-current bunch lengths, its predictions remain to be corrected for impedance-induced bunch lengthening, which is expected to remain approximately constant along the train.

\subsubsection{Investigation of luminosity deficit in Fall 2005}

For several months in 2005, PEP-II observed a significant, systematic variation of the luminosity along the bunch train. This is illustrated in Fig. 14 (top), where the rate of reconstructed $e^{+} e^{-}, \mu^{+} \mu^{-}$events is displayed as a function of where along the train the event occurred. The event rate, equivalent to the specific luminosity $\mathcal{L}_{s p}$ because the bunch currents are uniform within about $2 \%$ RMS, drops by $\sim 15 \%$ from the head to the tail of each mini-train.

Extensive studies were performed to understand these observations, which accounted for a $\sim 10 \%$ deficit in average luminosity. In the particular dataset presented here, the longitudinal lu-

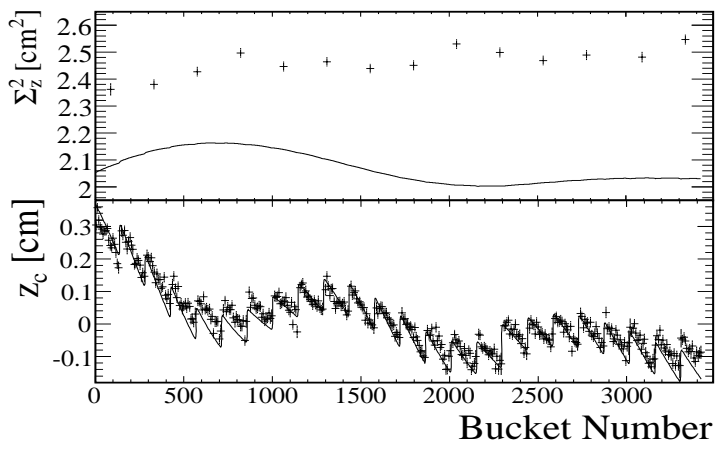

Figure 13. Bunch-number dependence of $\Sigma_{z}^{2}$ (top) and of the longitudinal luminous centroid (bottom) along a full train. Luminous-region data (points with error bars) are compared to the prediction (solid line) of an analytical RF model.

minous centroid (Fig. 14, center) varies by $5 \mathrm{~mm}$ peak-to-peak along the full train, reflecting the differential RF-phase transient between the $e^{+}$ and $e^{-}$rings. A $1-2 \mathrm{~mm}$ variation occurs within each mini-train; geometrical considerations (that ignore beam-beam effects, if any) limit its predicted impact on $\mathcal{L}_{s p}$ to less than $2 \%$. The most statistically significant signal is a $2 \mu \mathrm{m}$ variation of $\sigma_{x \mathcal{L}}$ along the mini-train (Fig. 14, bottom), which, depending on the $e^{+} / e^{-}$IP spot size ratios, might explain most of the luminosity drop. Unfortunately, the statistical precision of the vertical luminous-size measured along the mini-train using the method of Section 6, remains insufficient to reveal any systematic degradation of the vertical IP spot size, as might be induced for instance by electron-cloud effects. Studies of variations in $\Sigma_{z}$ or $\beta_{y \text {,eff }}^{*}$ along the train provide no additional clue. Empirical tuning ultimately eliminated most of the $\sigma_{x \mathcal{L}}$ and $\mathcal{L}_{s p}$ variation along the train. 

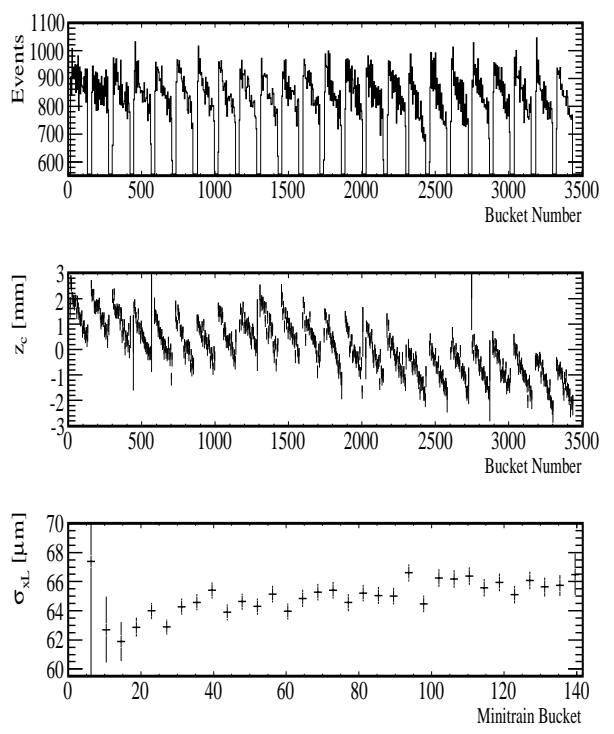

Figure 14. Variation of the number of $e^{+} e^{-}$, $\mu^{+} \mu^{-}$events (top) and of the longitudinal luminous centroid $z_{c}$ (center) along the full bunch train. The abort gap occurs at the right edge of the plots. Bottom: variation of the horizontal luminous size along one mini-train, averaged over all mini-trains.

\section{Longitudinal Dependence of the Trans- verse Luminous Size}

\subsection{Methodology}

The vertical hourglass effect manifests itself both by a distortion of the longitudinal luminosity profile, analyzed in Sec. 5.1 above, and by the growth of the vertical luminous size $\sigma_{y \mathcal{L}}$ with increasing distance from the vertical waist. While the longitudinal-profile analysis is relatively straightforward in terms of instrumental performance, transverse luminous-size measurements require an exacting control of the tracking resolution, which in the vertical plane is several times larger than the RMS width of the luminous region one attempts to measure.

The combination of the two methods was originally exploited by Cinabro et al.[1], who used $e^{+} e^{-} \rightarrow \mu^{+} \mu^{-}$events recorded by the CLEO detector. These authors performed a simultaneous fit to the $z$-dependence of the luminosity and of the vertical luminous size to extract the tracking resolution, the bunch length, the vertical emittance and $\beta_{y}^{*}$ at the CESR IP. A crucial ingredient in their approach was that, in the CLEO detector and within the then-available statistical precision, the luminous-size resolution was considered essentially independent of the position of the event vertex: it could therefore be directly identified with the minimum of $\sigma_{y \mathcal{L}}(z)$. The resulting correlation between the resolution, the fitted value of $\epsilon_{y}$ and that of $\beta_{y}^{*}$ was lifted using the longitudinal luminosity distribution.

The principle of the original measurement of $\sigma_{y \mathcal{L}}$ ran as follows. In the limit of a perfect detector, and for muons emitted close to the horizontal plane $(\phi \approx 0, \pi)$, the distribution of their distance of closest approach (DOCA) to the beam axis reflects the vertical size of the luminous region. Binning the DOCA distribution (corrected for detector resolution) of quasi-horizontal muons as a function of the longitudinal position of the $\mu^{+} \mu^{-}$vertex, thus measures the vertical hourglass shape. Similarly, the DOCA distribution of quasi-vertical muons $(\phi \approx \pm \pi / 2)$ measures the horizontal luminous size (but remains insensitive to the horizontal hourglass effect because $\beta_{x}^{*} \gg \sigma_{z}$ ). While conceptually less straightforward than simply vertexing the two muon tracks, the DOCA method has the major advantage that its resolution is self-calibrating (as will be shown below) and at least as good as that of the vertexing method.

The analysis reported in the present Section exploits, as did Ref. [1], the azimuthal dependence of the transverse impact parameter of the $\mu^{ \pm}$to the beam axis. The statistical sensitivity of the measurement is enhanced by performing maximum-likelihood fits to the full azimuthal and longitudinal dependence of the DOCA distribution.

In contrast to the CLEO report however, an early study [31] found that in BABAR and with the very large event samples afforded by the $B$ Factory, assuming a uniform resolution would be highly inadequate and lead to severe biases. We 
therefore introduce a technique in which detectorresolution estimates for each event are used in conjunction with detailed resolution maps obtained from the data themselves. This approach allows one to extract the vertical IP $\beta$-function and effective emittance from the measurement of $\sigma_{y \mathcal{L}}(z)$ alone, thereby providing a measurement independent of, and complementary to, that obtained from the longitudinal luminosity distribution.

This chapter is organized as follows. The accessible beam parameters and the related formalism were already presented in Sec. 3.4. The experimental determination of the distance of closest approach and of its event-by-event error is detailed in Sec. 6.2 below. Section 6.3 is devoted to the extraction of the resolution maps from the data. The measurement of the horizontal and vertical luminous size, the fitting technique, the accompanying validation studies and the estimation of the systematic uncertainties are presented in Sec. 6.4. Finally, the technique is applied to archived BABAR data to display the long-term evolution of the horizontal $\left(\sigma_{x \mathcal{L}}\right)$ and vertical $\left(\sigma_{y \mathcal{L}}\right)$ luminous sizes, and of the vertical IP $\beta$-function, effective waist position and effective emittance.

\subsection{Fundamental Observables}

$e^{+} e^{-} \rightarrow \mu^{+} \mu^{-}$events are reconstructed as described in Sec. 2.2.3. The Kalman fit of each muon returns the five helix parameters of the track and an error matrix. These fits are carried out in a coordinate system defined by the $B A B A R$ drift chamber. In order to extract the luminous sizes, the reconstructed tracks are then translated and rotated into the coordinate system defined by the luminous ellipsoid; the error matrices are transformed accordingly.

The key observables are shown in Fig. 15. For each track we fit the point of closest approach (POCA) in the transverse plane to the beam axis. The signed distances from each POCA to the beam axis are called distances of closest approach and denoted by $d_{1}$ and $d_{2}$. We also reconstruct the azimuthal angle of each track $\left(\phi_{1}, \phi_{2}\right)$, and their polar angle with respect to the beam axis $\left(\theta_{1}, \theta_{2}\right)$. The $z$-coordinate $z_{i}(i=1,2)$ of each muon at the event vertex is defined as the value of $z$ along the corresponding track in the detector coordinate system, at the point of closest approach. The uncertainties on $d_{1}$ and $d_{2}$, from the error matrices of the track-reconstruction fits, are called $\delta_{1}$ and $\delta_{2}$.

Based on the variables above, several additional quantities are defined to characterize an event. We estimate the DOCA of the actual event vertex using the average DOCA of the two muons, $d \equiv\left(d_{1}-d_{2}\right) / 2$. We also define the "miss distance" $m$ as half the distance between the POCAs in the $x$ - $y$ plane: $m \equiv\left(d_{1}+d_{2}\right) / 2$ (note that $m$ is zero for a perfectly reconstructed event). The total error on $d$ is estimated by $\delta \equiv \sqrt{\delta_{1}^{2}+\delta_{2}^{2}} / 2$, which is mathematically identical to the error on $m$. The longitudinal position of the $\mu^{+} \mu^{-}$vertex is calculated as the weighted average $z_{v} \equiv$ $\left(z_{1} \tan \lambda_{2}+z_{2} \tan \lambda_{1}\right) /\left(\tan \lambda_{2}+\tan \lambda_{1}\right)$, where the dip angle $\lambda$ is the complement of the polar angle $(\lambda=\pi / 2-\theta)$.

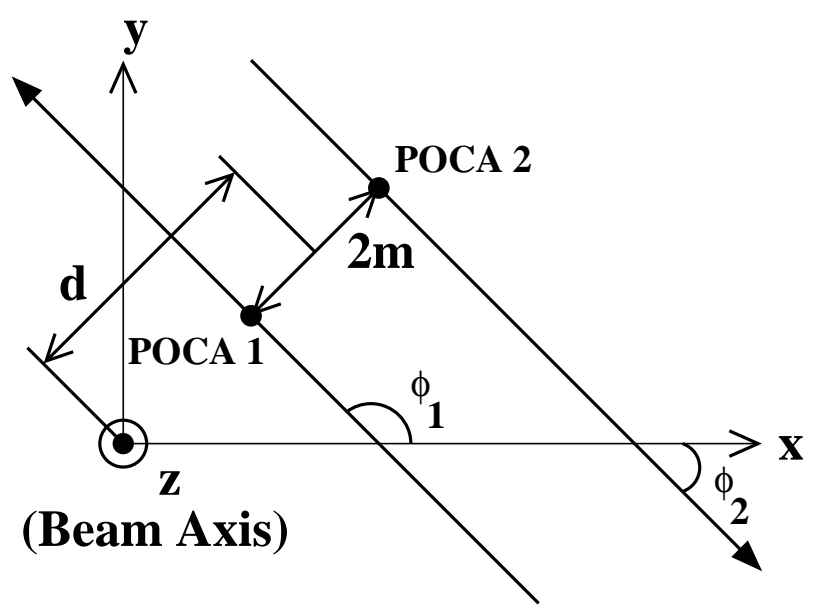

Figure 15. Schematic view of two reconstructed muon tracks in the transverse plane. Each track has a reconstructed point of closest approach to the beam axis (POCA), from which the average DOCA $d$ and the miss distance $m$ are calculated. 


\subsection{Resolution Model}

\subsubsection{Single-event Resolution Estimator}

The DOCA error $\delta$ is an estimate of the resolution for one given event. For an ensemble of events, the width of the miss-distance distribution (Fig. 16) provides a direct measurement of the actual resolution in the data. Figure 17 shows the width $\sigma_{m}$ of this distribution as a function of $\delta$ : the two quantities are tightly, and linearly, correlated over a wide range. Therefore we can use the per-event error $\delta$ as a reliable estimate of the uncertainty affecting the transverse vertex position in that particular event. A detailed study of the $\sigma_{m}-\delta$ correlation reveals, however, that for very large values of $\delta$, this variable no longer provides a good estimate of the miss-distance resolution [32]. Events with $2 \delta>35 \mu \mathrm{m}$ are therefore excluded from the data sample.

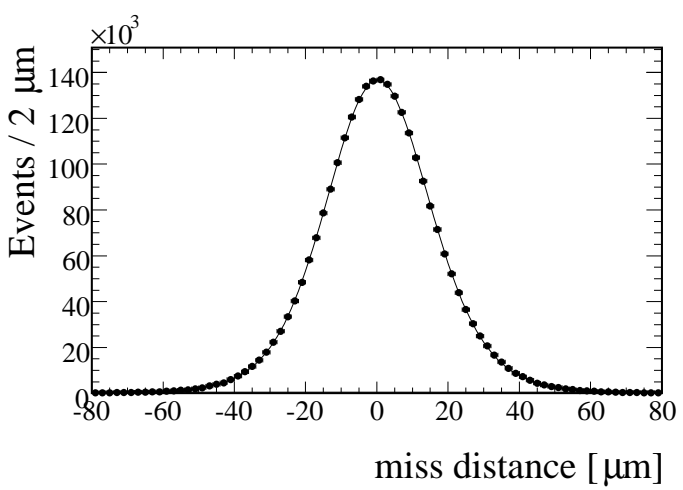

Figure 16. Distribution of the miss distance $m$ for a typical data sample. The curve is the global fit to the resolution function of Eq. 20.

The $\phi$ - and $z$-dependence of the DOCA error are presented in Fig. 18. The strong variation of the resolution with azimuthal angle is a direct consequence of the structure of the SVT (Sec. 2.2.1). Those muons traversing a section of the inner two layers with a finer readout pitch, exhibit a significantly smaller vertexing uncertainty. The error also depends on the distance from the

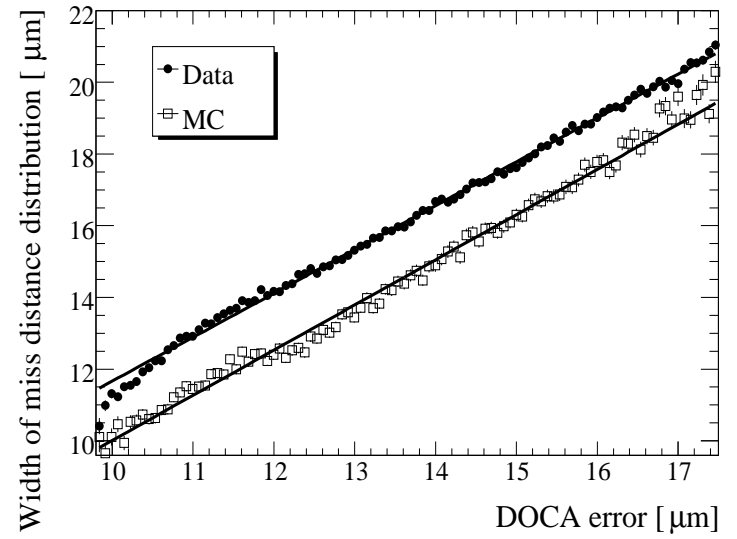

Figure 17. Width $\sigma_{m}$ of the miss-distance distribution, as extracted from Gaussian fits to real (full circles) or simulated (open squares) data, as a function of the DOCA error $\delta$. The lines are linear fits to the points.

event vertex to the first layer of the SVT, as reflected by the hexagonal periodicity of Fig. 18a. In contrast to what was reported for CLEO [1], we also observe a significant $z$-dependence of the resolution (Fig. 18b), which must imperatively be taken into account to avoid biasing the measured longitudinal dependence of the vertical luminous size.

\subsubsection{Event-by-event Resolution Function}

While the DOCA error $\delta$ provides a good eventby-event estimate of the actual resolution, the slope of the linear fits in Fig. 17 is noticeably larger than 1 . To provide a quantitatively accurate estimate of the resolution in a given event, $\delta$ must therefore be scaled by an appropriate factor, that will be obtained from the data themselves.

We translate from $\delta$ to the measured resolution using a resolution function, $\mathcal{R}$, constructed as the sum of three Gaussian distributions $G_{i}$ :

$$
\begin{aligned}
\mathcal{R}(d) & =f_{1} G_{1}\left(d ; \mu_{1}(\delta), S_{1}(\delta) \delta\right) \\
& +f_{2} G_{2}\left(d ; \mu_{2}, S_{2} \delta\right) \\
& +f_{3} G_{3}(d ; 0,62.5 \mu \mathrm{m}) .
\end{aligned}
$$



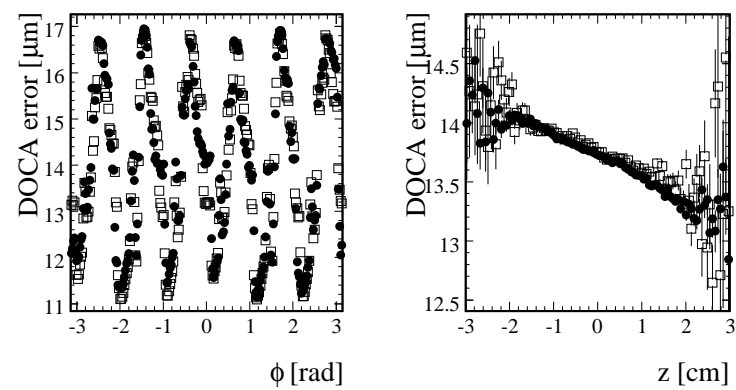

Figure 18. DOCA error $\delta$ as a function of (left) the azimuthal angle $\phi$ of the muon pair, and (right) the longitudinal position $z_{v}$ of the $\mu^{+} \mu^{-}$ vertex, for data (full circles) and for simulation (open squares).

Here the coefficients $f_{i}$ represent the fraction in each component, and are constrained by $f_{3}=$ $1-f_{2}-f_{1}$. The distributions have means $\mu_{i}$, and widths that include scale factors $S_{i}$. Although this parameterization of the resolution gives a good fit to the overall miss distance distribution (Fig. 16), it remains insufficient to fully describe the detector resolution; in particular, the scale factors vary significantly as a function of the vertex position and direction of the muons. The actual resolution functions are therefore determined in two steps, as follows.

We first determine the fractions $f_{i}$ by fitting a large event sample (a minimum of one month of data-taking) to the resolution function of Eq. 20, with means $\mu_{i}$ and scale factors $S_{i}$ that do not depend explicitly on the direction and production point of the muons. To achieve a good fit, it proved necessary at this stage to split the mean and scale factor of the core Gaussian $G_{1}$ as a function of $\delta: \mu_{1}$ and $S_{1}$ have separate values for events with $2 \delta<25 \mu \mathrm{m}$ and events with $2 \delta>25 \mu \mathrm{m}$.

In a second step, we split the data in bins of the vertex position $z_{v}$, azimuth $\phi_{1}$ and polar angle $\theta_{1}$ of the leading muon. In each bin, we refit the miss-distance distribution to Eq. 20, with the fractions $f_{i}$ fixed to the values found in the first step and without splitting $\mu_{1}$ and $S_{1}$ by the value of $\delta$. This yields the final scale factors $S_{i}$ and means $\mu_{i}$ that define the resolution function we will use for extracting the beam parameters from the measured DOCA distribution. The result of this procedure is illustrated in Fig. 19 for the scale factor $S_{1}$ of the core Gaussian, revealing up to $30 \%$ peak-to-peak variations in the width of the resolution function.

\subsection{Transverse Luminous Size Measure- ments}

\subsubsection{Fitting Procedure}

The Gaussian probability density function $(\mathrm{PDF}) \mathcal{P}$, that describes the DOCA distribution for a perfect detector, can be written as

$$
\begin{aligned}
& \mathcal{P}(d, \phi, z)= \\
& \quad \exp \left(-\frac{1}{2} \frac{\left[d-\left(y_{0}(z) \cos \phi_{t}-x_{0} \sin \phi_{t}\right)\right]^{2}}{\left[\sigma_{y \mathcal{L}}(z) \cos \phi_{t}\right]^{2}+\left[\sigma_{x \mathcal{L}} \sin \phi_{t}\right]^{2}}\right) .
\end{aligned}
$$

Here the transverse luminous sizes $\sigma_{y \mathcal{L}}$ and $\sigma_{x \mathcal{L}}$ are simply related to the single-beam spot sizes in the vicinity of the IP (Eq. 8); the $z$-dependence of $\sigma_{y \mathcal{L}}$ is controlled by the vertical hourglass parameters; and the variable $\phi_{t} \equiv \phi-t_{x y}$ allows for a global rotation of the luminous ellipsoid around the beam axis by an angle $t_{x y}$, with respect to the detector coordinate system. The parameters $x_{0}$ and $y_{0}$ account for medium-term drifts of the luminous centroid, and the $z$-dependence of the vertical centroid:

$y_{0}(z)=y_{0}(z=0)+\left(d y_{0} / d z\right) z$

reflects the fact that the collision axis is tilted vertically by 1 to $2 \mathrm{mrad}$ (see Fig. 4 , top).

To extract these IP parameters, we perform an unbinned maximum-likelihood fit of the measured DOCA distribution to the convolution of the DOCA PDF above with the detector resolution $\mathcal{R}$. This fitting function $\mathcal{P}(d, \phi, z) \otimes \mathcal{R}(d)$ depends on the resolution parameters $\left(f_{i}, \mu_{i}, S_{i}\right)$, which are kept fixed to the values determined in Sec. 6.3.2, and on the following beam parameters which are left free in the fit:

- the mean transverse position $x_{0}$ and $y_{0}$ of the luminous centroid, and the mean vertical luminous tilt $d y_{0} / d z$; 
- the $z$-averaged horizontal luminous size $\sigma_{x \mathcal{L}}$

- the vertical luminous size at the waist, $\sigma_{y \mathcal{L}}\left(z=z_{y}^{w}\right)$

- the longitudinal position $z_{y}^{w}=z_{y+}^{w}=z_{y-}^{w}$ of the (common) vertical waist;

- the (common) vertical IP $\beta$-function $\beta_{y, D}^{*}$ $=\beta_{y+}^{*}=\beta_{y-}^{*}$, which determines the $z$ dependence of $\sigma_{y \mathcal{L}}$;

- the rotation $t_{x y}$ of the luminous ellipsoid around the collision axis.

Under the assumptions that the vertical $\beta$ functions and the location of the vertical waist are the same for the two beams, $\sigma_{y \mathcal{L}}(z)$ is given by

$$
\begin{aligned}
\sigma_{y \mathcal{L}}(z) & =\sqrt{\frac{\epsilon_{y, \mathrm{eff}} \beta_{y, D}^{*}}{2}\left[1+\left(\frac{z-z_{y}^{w}}{\beta_{y, D}^{*}}\right)^{2}\right]} \\
& =\sigma_{y \mathcal{L}}\left(z=z_{y}^{w}\right) \sqrt{1+\left(\frac{z-z_{y}^{w}}{\beta_{y, D}^{*}}\right)^{2}}
\end{aligned}
$$

where the final expression is the one used in our fit.

Figure 20 shows the measured DOCA distribution, overlaid with the fit results, for three different cases. A typical data sample is shown on the top; the overall fit result is shown in blue, while the red curve shows the contribution of the resolution to the shape of the distribution. The subset of quasi-vertical muons (center) is primarily sensitive to the horizontal luminous size. The overall fit result for those events is shown in blue, and the contribution to that curve from the horizontal size only, ignoring the vertical size and resolution, appears in green: it totally determines the shape of this distribution. Finally, quasihorizontal muons (bottom) determine the vertical luminous size. Here the blue curve is defined as in the other two plots, while the magenta curve displays the contribution of the vertical luminoussize size only, ignoring horizontal size and instrumental resolution; the difference between the blue and magenta curves reflects the combined contributions, for these muons, of the resolution and of the horizontal luminous size.

As a check on the self-consistency of the fitting procedure, we refit the data in slices of $z$ and compare the results to those of the global fit above, revealing an excellent internal agreement (Fig. 21). The vertical hourglass shape is apparent, and highly reproducible from one dataset to the next. The horizontal luminous size is consistent with a constant within $<0.5 \mu \mathrm{m}$ RMS: the expected horizontal hourglass effect is of comparable magnitude, and therefore unlikely to be detectable.

The values of $\beta_{y, D}^{*}$ and $\sigma_{y \mathcal{L}}\left(z=z_{y}^{w}\right)$ are highly correlated in the fits. Figure 22, which shows the error contours for the aforementioned parameters for a typical fit, illustrates this correlation.

\subsubsection{Validation Studies}

The fitting procedure was validated using a large number of simulated ("toy") experiments, where event samples are generated using the same PDFs and resolution functions as those subsequently used for fitting. No convergence problems nor significant fit biases were found during those checks.

More sensitive tests were then carried out using a GEANT-4 simulation that incorporates the full response of the BABAR detector. In a first step, ten different simulated data samples were produced, with generated $\sigma_{y \mathcal{L}}$ values ranging from 2 to $20 \mu \mathrm{m}$ but constant with $z$ (i.e. ignoring the hourglass effect). Comparing the "measured" vertical luminous size returned by the fit $\left(\sigma_{y \mathcal{L}_{\text {fit }}}\right)$ to its "true" value $\left(\sigma_{y} \mathcal{L}_{\text {generated }}\right)$ reveals a systematic bias (Fig. 23). A simple fit to

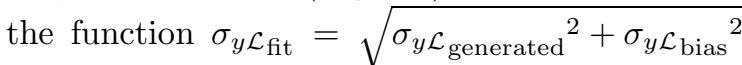
yields $\sigma_{y \mathcal{L}_{\text {bias }}}=2.0 \pm 0.3 \mu \mathrm{m}$. This "bias correction" must be applied to the fitted value of $\sigma_{y \mathcal{L}}$ to properly reproduce the true (generated) luminous size. No such bias is present when analyzing the parameterized ("toy") simulations mentioned above, suggesting that the problem is somehow related to the detailed description of the geometry or of the material of the BABAR detector. The source of this effect remains unknown, and 
a $z$-independent $2 \mu \mathrm{m}$ subtraction is applied in quadrature to all subsequent $\sigma_{y \mathcal{L}}$ fit results. Empirical as it may be, this approach yields vertical luminous-size measurements that are fairly consistent with those of the boost method, as will be shown in Sec. 9.

In a second step, simulated dimuon samples were generated using realistic emittance values and vertical-hourglass parameters, and analyzed using a procedure identical to that applied to real data (including the bias correction above). The results of this validation are presented in Table 4 . The fitted values of $\beta_{y}^{*}$ agree with the generated input within statistical errors.

These studies highlight the fundamental importance of the very detailed parameterization of the resolution function introduced in Sec. 6.3.2, and that was used to produce the results shown in Table 4. If we fit the same simulated samples using a simpler resolution model that includes only global scale factors (i.e. that does not account for the variations shown in Fig. 19), the fit results for $\beta_{y, D}^{*}, z_{y}^{w}$ and $t_{x y}$ exhibit significant biases.

Table 4

Fit results on simulated event samples (full detector simulation). The errors are statistical only.

\begin{tabular}{l|rr} 
Parameter & Generated & Fit result \\
\hline \hline & \multicolumn{2}{|c}{ Sample 1: 1,172,222 events } \\
\hline$\beta_{y+}^{*}=\beta_{y-}^{*}(\mathrm{~cm})$ & 1.21 & $1.16 \pm 0.13$ \\
$\sigma_{y \mathcal{L}}\left(z=z_{y}^{w}\right)(\mu \mathrm{m})$ & 3.25 & $3.55 \pm 0.17$ \\
$z_{y}^{w}(\mathrm{~cm})$ & -0.9 & $-0.99 \pm 0.08$ \\
$t_{x y}(\mathrm{mrad})$ & 0 & $0.33 \pm 0.54$ \\
$\sigma_{x \mathcal{L}}(\mu \mathrm{m})$ & 75.76 & $76.26 \pm 0.06$ \\
\hline \hline & \multicolumn{2}{|c|}{ Sample 2: 1,336,813 events } \\
\hline$\beta_{y+}^{*}=\beta_{y-}^{*}(\mathrm{~cm})$ & 0.80 & $0.88 \pm 0.07$ \\
$\sigma_{y \mathcal{L}}\left(z=z_{y}^{w}\right)(\mu \mathrm{m})$ & 2.64 & $3.12 \pm 0.15$ \\
$z_{y}^{w}(\mathrm{~cm})$ & -0.9 & $-0.84 \pm 0.06$ \\
$t_{x y}(\mathrm{mrad})$ & 0 & $0.11 \pm 0.51$ \\
$\sigma_{x \mathcal{L}}(\mu \mathrm{m})$ & 75.76 & $76.17 \pm 0.06$
\end{tabular}

The potential impact of beam-beam-induced distortions was investigated using the same strong-strong simulation as in Sec. 5. At the low- est bunch currents, the "true" values of $\sigma_{y \mathcal{L}}(z=$ $\left.z_{y}^{w}\right)$ and of $\beta_{y, D}^{*}$ are reproduced to better than $0.01 \mu \mathrm{m}$ and $0.1 \mathrm{~mm}$ respectively. At high bunch currents, where the simulated vertical luminosity distribution displays distinctly non-Gaussian tails, the luminous size at the waist is nevertheless reproduced to better than $0.2 \mu \mathrm{m}$, and $\beta_{y, D}^{*}$ decreases by $\sim 0.4 \mathrm{~mm}$, which is consistent with the result extracted, in Sec. 5.1.3, from the longitudinal luminosity profile using the same simulated sample.

\subsubsection{Systematic Uncertainties}

The main source of systematic uncertainty is the $\sim 2 \mu \mathrm{m}$ bias correction, which because of the intrinsic correlation between $\sigma_{y \mathcal{L}}\left(z=z_{y}^{w}\right)$ and $\beta_{y, D}^{*}$, affects both of these parameters. We estimate this uncertainty by varying the correction by $\pm 1 \mu \mathrm{m}$ and refitting the data. The corresponding variations in $\sigma_{y \mathcal{L}}$ and $\beta_{y, D}^{*}$ depend on the central values of both parameters, and result in asymmetrical error bars as illustrated in Figs. 24 and 25. In most cases, the systematic uncertainty on each parameter is comparable to, or slightly larger than, the corresponding statistical error.

We use a standard BABAR procedure for estimating uncertainties due to possible misalignment of the SVT. Simulated events are reconstructed using a variety of alignment configurations which purposely introduce errors in the alignment. We then repeat fits to these samples to estimate the uncertainty introduced by alignment errors. This method is intended to set an upper limit on the possible error. We find that in the worst case, misalignment increases the measured values of $\beta_{y, D}^{*}$ by about $20 \%$ and of $\sigma_{y \mathcal{L}}$ $\left(z=z_{y}^{w}\right)$ by about $30 \%$.

Additional systematic errors could be introduced by short-term drifts of the transverse luminous centroid, that would remain unaccounted for by the medium-term average parameters $x_{0}$ and $y_{0}$ (see Sec. 6.4.1). For instance, rapid variations in the actual vertical centroid could, if large enough, bias the $\sigma_{y \mathcal{L}}$ and $\beta_{y, D}^{*}$ measurement. We have studied several weeks of data, binned in one-day intervals, and concluded that the transverse centroid motion is typically slow enough and small enough for the associated systematic errors 
to be neglected.

\subsection{History of Transverse Luminous Sizes and Vertical $\beta$-function at the PEP-II IP}

The history of the IP parameters extracted from the dimuon DOCA distribution over about eighteen months of BABAR running, are presented in Figs. 24 to 28. The vertical luminous size (Fig. 24) varies from 2 to $6 \mu \mathrm{m}$, corresponding to $e^{ \pm}$IP spot sizes of 3 to $9 \mu \mathrm{m}$, and reasonably consistent with PEP-II design parameters (Table 1). The $\beta_{y}^{*}$ measurements extracted from the longitudinal dependence of $\sigma_{y \mathcal{L}}$ are consistent with the equivalent quantity computed from uncoupled lattice functions measured in single-bunch mode by the phase-advance method (Fig. 25). Combining the measured values of $\beta_{y, D}^{*}$ and $\sigma_{y \mathcal{L}}$ yields, under the assumption that $\beta_{y+}^{*}=\beta_{y-}^{*}$, an estimate of the effective vertical emittance (Eq. 11), measured in collision and at nominal beam currents, i.e. including the impact of the actual beam-beam-induced emittance blowup. The resulting time history (Fig. 26) indicates $e^{ \pm}$emittances of a few $\mathrm{nm}$, consistent with the design goal of $2-3 \mathrm{~nm}$.

The history of the horizontal luminous size $\sigma_{x \mathcal{L}}$ is displayed in Fig. 27; the results are consistent with expectations, provided the dynamic- $\beta$ effect is taken into account. No convincing signature of an horizontal hourglass effect was found in the data, as expected from the large value of $\beta_{x}^{*} / \sigma_{z \pm}$. Finally, Fig. 28 shows the history of the azimuthal tilt of the luminous region around the beam axis. The data establish the presence, beyond any statistical doubt, of significant $x-y$ coupling throughout the data-taking period; the observed tilt is consistent, in sign and magnitude, with that of the luminosity ellipse (not to be confused with the luminous ellipsoid) reconstructed by transverse beam-beam scans at different angles (see e.g. Sec. 5 of Ref. [12]).

Longer-term histories of the vertical hourglass parameters, a comparison of the transverse IP spot sizes and emittances measured by this and other methods, as well as a brief discussion of the impact of $x-y$ coupling on luminous-region parameters, will be presented in Sec. 9.

\section{Characterization of the Colliding-Beam Phase Space Using the Transverse- Boost Distribution of Muon Pairs}

The angular distribution of $\mu^{+} \mu^{-}$pairs was first exploited at CESR [33], in conjunction with the transverse luminous size, to estimate the $e^{ \pm}$ emittance and IP $\beta$-function. Here the technique is generalized to the case of asymmetric colliders, and its diagnostic reach extended by exploiting the spatial dependence of the boost-angle distributions. After presenting the measurement technique in Sec. 7.1 below, we apply the formalism of Sec. 3.5 to the characterization of the vertical (Sec. 7.2) and horizontal (Sec. 7.3) phase space at the PEP-II IP.

\subsection{Measurement Technique \\ 7.1.1. Single-event Observables}

The angular spread of the beams sets the scale for the measurement resolution required to extract beam parameters from the angular distribution of the boost vector B. Taking design PEP-II parameters as a guide (Table 1), suggests that the systematic uncertainty on this resolution should not exceed about $0.3 \mathrm{mrad}$.

The process $e^{+} e^{-} \rightarrow \mu^{+} \mu^{-}$produces finalstate muons with transverse momenta in the 3 to $5 \mathrm{GeV}$ range, resulting in an excellent angular resolution but a poor momentum resolution [5]:

$$
\begin{aligned}
\sigma_{\theta, \phi} & =\frac{1.0(\mathrm{mrad})}{p_{T}(\mathrm{GeV} / \mathrm{c})} \approx 0.25 \mathrm{mrad} \\
\frac{\sigma_{p_{T}}}{p_{T}} & =0.45 \%+0.13 \% \times p_{T}(\mathrm{GeV} / \mathrm{c}) \approx 1.0 \%
\end{aligned}
$$

for the individual muon tracks. Simply calculating the boost $\mathbf{B}$ as the vector sum of the two muon momenta results in boost-angle measurements with a wide range of resolutions averaging about $6 \mathrm{mrad}$, because of the resolution-limited track-curvature measurements.

The decay plane is defined by the $\mu^{+} \mu^{-}$momentum vectors (Fig. 29). While the direction of $\mathbf{B}$ within this plane is poorly determined because of the relatively large uncertainties affecting the magnitude of the individual muon momenta, the orientation of the decay plane itself is not affected by curvature-measurement errors. We therefore limit ourselves to measuring the event-by-event 
orientation of the vector $\hat{n}$ normal to this plane, defined by

$\hat{n}=\frac{\hat{p}_{\mu^{+}} \times \hat{p}_{\mu^{-}}}{\left\|\hat{p}_{\mu^{+}} \times \hat{p}_{\mu^{-}}\right\|}$.

The dip angle $\lambda_{n}$ and azimuthal angle $\phi_{n}$ of this vector are related to the boost angles $\left(x_{\mathrm{B}}^{\prime}, y_{\mathrm{B}}^{\prime}\right)$ of the parent $e^{+} e^{-}$pair by

$\tan \lambda_{n}=-x_{\mathrm{B}}^{\prime} \cos \phi_{n}-y_{\mathrm{B}}^{\prime} \sin \phi_{n}$.

The typical single-event dip-angle resolution is $0.6 \mathrm{mrad}$, and is affected by low enough a systematic uncertainty to measure the interesting spatial-dependent effects.

\subsubsection{Event Selection}

The dimuon events used in this analysis satisfy selection criteria similar to those listed in Sec. 4, but slightly tightened to maintain reliable covariance-matrix estimates for the track parameters. The events must contain two reconstructed tracks, each with at least 6 SVT hits and $28 \mathrm{DCH}$ hits; the forward-most track must lie well within the tracking acceptance $(\tan \lambda<2.5)$. The two-body invariant mass must satisfy $10.3<$ $M_{\mu \mu}<11.0 \mathrm{GeV}$, and the total calorimeter energy remain inconsistent with that of a Bhabha event $\left(E_{c a l}<3 \mathrm{GeV}\right)$. Cosmic-ray contamination is eliminated by a cut similar to that in Sec. 4 . The normal to the decay plane is reconstructed from the two muon tracks according to Eq. 23. The error on $\lambda_{n}$ is estimated from the covariance matrices of the reconstructed tracks and required not to exceed $0.8 \mathrm{mrad}$. To estimate the collision point, a common vertex is fit to the tracks and required to lie within a reasonable distance of the nominal interaction point $\left(\left|z_{v}\right|<5 \mathrm{~cm}\right.$, $\left.r_{v}<1 \mathrm{~cm}\right)$.

\subsubsection{Angular Distribution of the Boost Vector}

The collision point is first transformed to the luminous-region frame (the coordinate system defined by the luminous ellipsoid), by correcting for run-to-run shifts of the luminous centroid and for rotations of the average collision axis. An additional calibration fits the mean dip angle $\lambda_{n}$ as a function of $\phi_{n}$ (Fig. 30) according to Eq. 24, in order to determine the mean boost angles $\overline{x^{\prime}}{ }_{\mathrm{B}}, \overline{y^{\prime}}{ }_{\mathrm{B}}$ for each run and correct the event-by-event angles $\lambda_{n}$ for these short-term variations. The corrected dip-angle distributions are then binned in $\phi_{n}$, and each bin separately fit to extract its RMS spread $\sigma_{\lambda_{n}}$ accounting for detector resolution and radiation as described below. The resulting function of $\phi_{n}$ is fit (Fig. 31) to the function

$\sigma_{\lambda_{n}}^{2}=\sigma_{x_{\mathrm{B}}^{\prime}}^{2} \cos ^{2}\left(\phi_{n}\right)+\sigma_{y_{\mathrm{B}}^{\prime}}^{2} \sin ^{2}\left(\phi_{n}\right)$

to extract the horizontal and vertical boost angular spreads $\left(\sigma_{x_{\mathrm{B}}^{\prime}}, \sigma_{y_{\mathrm{B}}^{\prime}}\right)$.

The measured spread $\sigma_{\lambda_{n}}$ receives contributions from detector resolution, as well as from initial and final-state radiation:

- the geometrical layout of the BABAR silicon vertex tracker results in a $\phi$-dependent resolution, pictured in Fig. 32, and calculated by error propagation using the individual covariance matrices of the two tracks;

- initial-state radiation in $e^{+} e^{-} \rightarrow \mu^{+} \mu^{-}$ events also produces observable tails in the $\lambda_{n}$ distribution. Monte Carlo simulation of the $e^{+} e^{-} \rightarrow \mu^{+} \mu^{-}$process with initialstate radiation is used to create an exponential parameterization that reproduces these tails. The convolution of this exponential with a Gaussian that describes both the physical angular spread and the detector resolution, is then fitted to the $\lambda_{n}$ distribution. Accounting for initial-state radiation reduces the measured $\lambda_{n}$ spread by typically $0.05 \mathrm{mrad}$.

\subsubsection{Spatial Dependence of Boost Vari- ables}

The $z$-dependence of the boost angular spread is measured by extracting $\sigma_{x_{\mathrm{B}}^{\prime}}$ and $\sigma_{y_{\mathrm{B}}^{\prime}}$ as described above, in bins of the longitudinal position of the $\mu^{+} \mu^{-}$vertex. It is assumed that the optical waists do not move significantly over the duration of a single measurement; therefore the vertex $z$-positions are not corrected for short-term displacements of the collision point that could be caused, for instance, by RF-phase fluctuations.

The correlation between position and boost angle is measured by extracting the mean boost angle in bins of transverse vertex position within 
the luminous-region frame. For example, the $y-y^{\prime}$ correlation is measured by first binning events in $y$ and then, in each bin, fitting $\lambda_{n}$ versus $\phi_{n}$ for the mean angle $\overline{y^{\prime}}{ }_{\mathrm{B}}$. A linear fit to the resulting $y$-dependence of $\overline{y^{\prime}}{ }_{\mathrm{B}}$ (as suggested by Eq. 15) yields the slope $\delta \bar{y}^{\prime}{ }_{\mathrm{B}} / \delta y$. This process is repeated in bins of $z$ to measure the longitudinal variation of this slope, to which Eq. 16 can be fit. Fig. 33 shows simulations of the $x-\bar{x}^{\prime}{ }_{\mathrm{B}}$ and $y-\bar{y}_{\mathrm{B}}{ }_{\mathrm{B}}$ correlations with and without including the detector resolution, illustrating the impact of correlated errors in the estimated angle and point of origin of the tracks. In the case of the $x-\overline{x^{\prime}}{ }_{\mathrm{B}}$ correlation, tracking errors introduce an overall, $z$ independent shift in the measured correlation coefficient. For the $y-\bar{y}_{\mathrm{B}}{ }_{\mathrm{B}}$ correlation measurement, detector resolution additionally produces a strong dilution of the variation with $z$, since the vertexing resolution is considerably larger than the vertical beam sizes near the IP.

\subsection{Characterization of Vertical Phase Space}

The vertical hourglass effect leaves striking footprints in the spatial dependence of the vertical angular distribution of the boost vector, thereby providing measurements of the vertical emittance, IP $\beta$-function and optical-waist position (Sec. 7.2.1) in the presence of beam-beam effects (Sec. 7.2.2). These BABAR-based measurements, carried out during routine physics running, allow to reconstruct the long-term history of the $e^{+}$and $e^{-}$vertical phase space at the PEP-II IP (see Sec. 7.2.3).
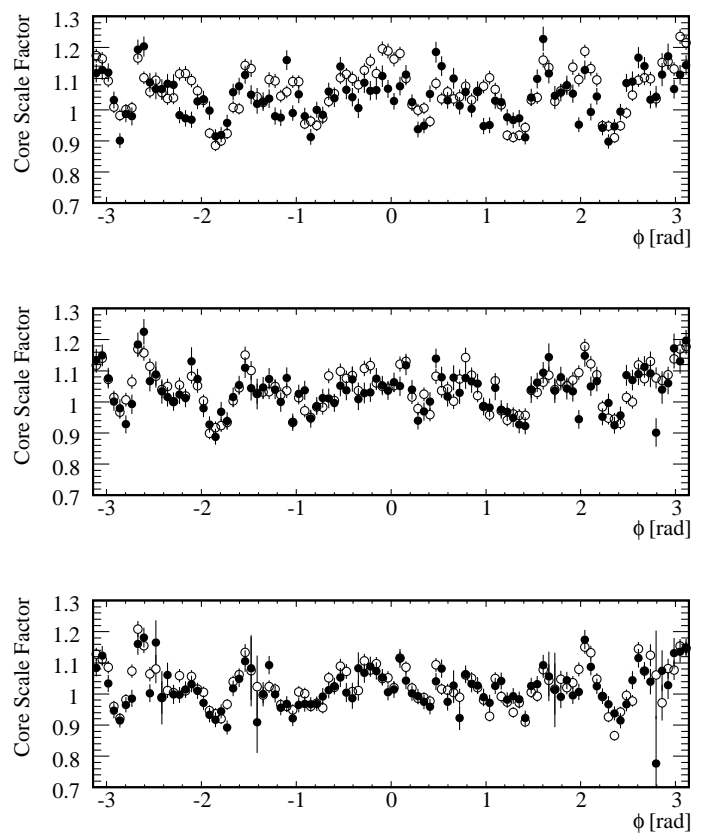

Figure 19. Scale factor $S_{1}$ of the core component of the resolution function as a function of $\phi_{1}$, from a fit of Eq. 20 to a representative data sample. The detector is binned in three bins of $\cos \theta$ : $\cos \theta_{1}<0.65$ (top), $0.65<\cos \theta_{1}<0.75$ (center), $0.75<\cos \theta_{1}<0.85$ (bottom); and three bins of $z_{v}$ : negative $z_{v}$ region (full circles), central $z_{v}$ region (not shown), positive $z_{v}$ region (open circles), where $z_{v}$ is measured in detector coordinates and the exact boundaries of each $z_{v}$ region depend on the data-taking period. The points for the central $z_{v}$ region are omitted for the sake of clarity; they typically fall between the full and open circles. 

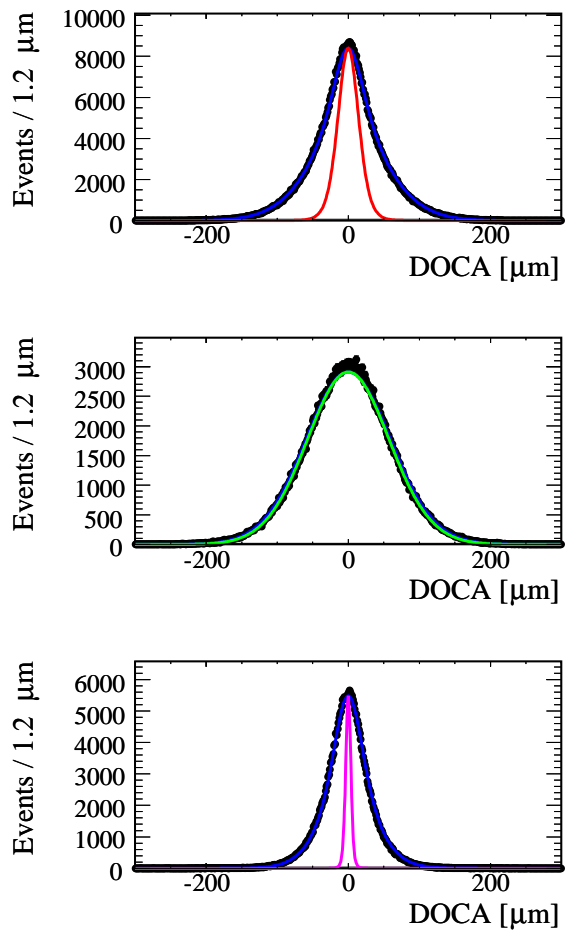

Figure 20. Measured distribution of $d$, the distance of closest approach to the beam line, for a typical data sample. Top: all muons; center: quasi-vertical muons $\left(\pi / 4<\left|\phi_{1}\right|<3 \pi / 4\right)$; bottom: quasi-horizontal muons $\left(\left|\phi_{1}\right|<\pi / 4\right.$, $\left.\left|\phi_{1}-\pi\right|<\pi / 4\right)$. The points are the data; the curves are described in the text.
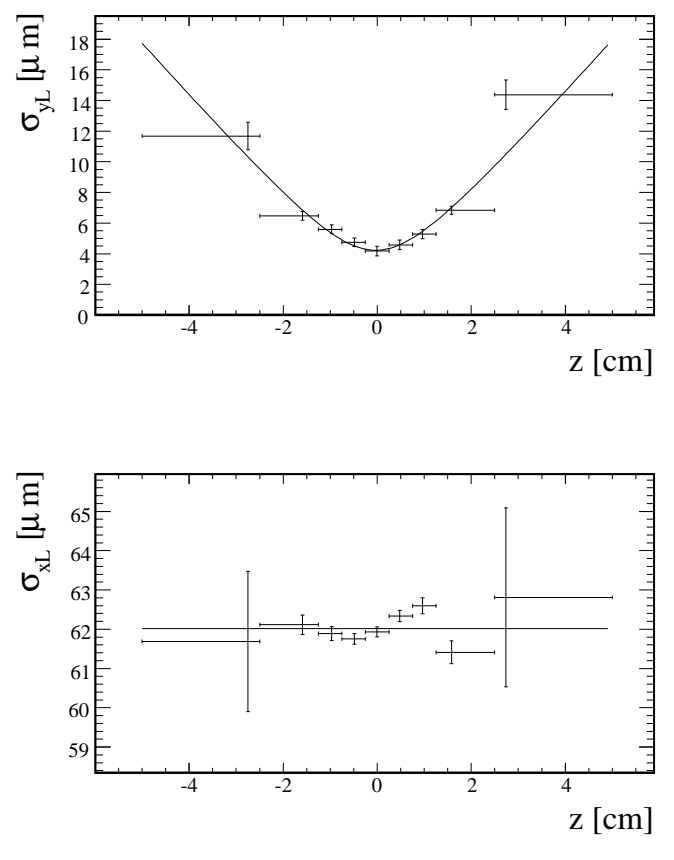

Figure 21. Measured $z$-dependence of the vertical (top) and horizontal (bottom) luminous size, extracted from a sample of $8.5 \times 10^{5} \mu^{+} \mu^{-}$events collected over ten days in December 2003. The lines show the result of the simultaneous fit to all events; the points with error bars result from fitting the data separately in each $z$ bin. 


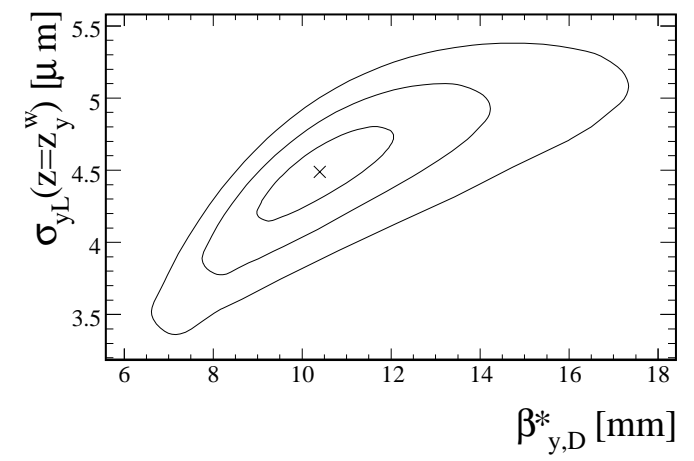

Figure 22. The inner, center, and outer curves show the boundaries of the 1,2 , and $3 \sigma$ regions allowed by the fit around the central value indicated by a cross. These results are from a typical fit to the data. The allowed regions are tilted due to the correlation between $\beta_{y, D}^{*}$ and $\sigma_{y \mathcal{L}}\left(z=z_{y}^{w}\right)$.

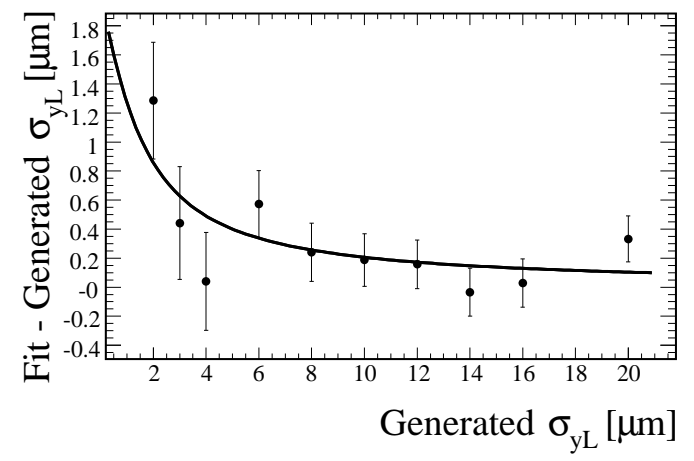

Figure 23. Difference between the fitted and the generated values of $\sigma_{y \mathcal{L}}$ in simulated event samples generated with no hourglass effect. The error bars reflect the statistics of the simulation. The curve fitted to the data points provides a parameterization of the measurement bias.

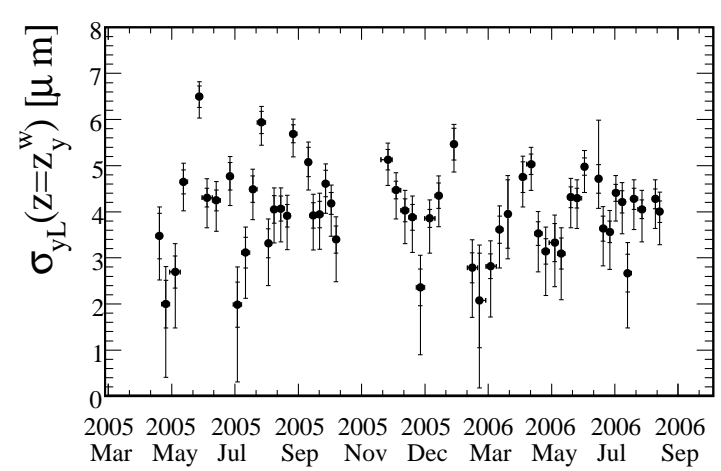

Figure 24. Medium-term history of the vertical luminous size at the waist, as measured using the dimuon DOCA distribution. The inner error bar is statistical only; the outer error bar is the sum (in quadrature) of the statistical error and of the systematic uncertainty associated with the bias correction. 


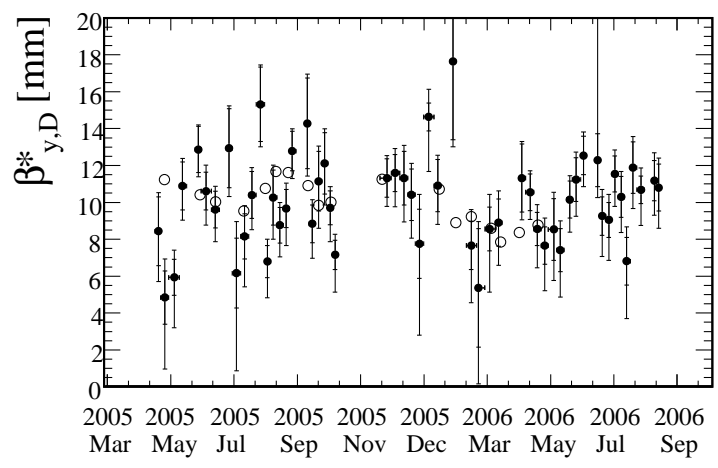

Figure 25. Medium-term history of the (assumed common) vertical IP $\beta$-function $\beta_{y, D}^{*}$, as measured using the DOCA distribution. The inner error bar is statistical only; the outer error bar is the sum (in quadrature) of the statistical error and of the systematic uncertainty associated with the bias correction. The open circles represent phase-advance measurements of $\beta_{y+,-}^{*}$, appropriately combined assuming $\epsilon_{y+}=$ $1.5 \mathrm{~nm} \mathrm{rad}, \epsilon_{\mathrm{y}-}=2.5 \mathrm{~nm}$ rad and neglecting $x-y$ coupling.

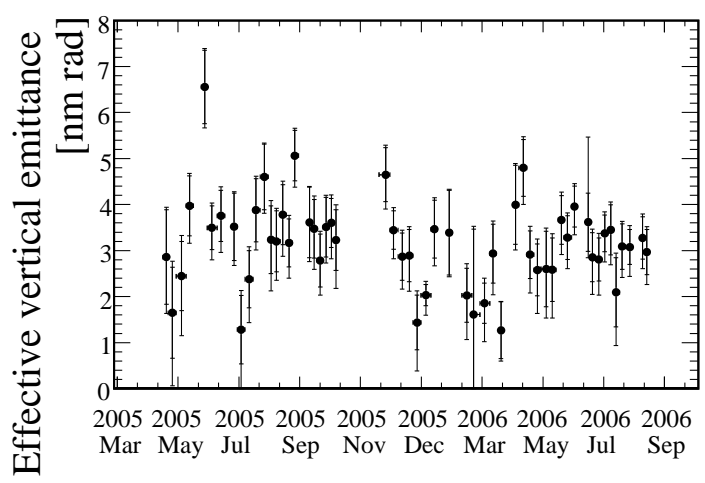

Figure 26. Medium-term history of the effective vertical emittance $\epsilon_{y \text {,eff }}$, as inferred from the values of $\sigma_{y \mathcal{L}}$ and $\beta_{y, D}^{*}$ extracted from the DOCA distribution. The inner and outer error bars have the same meaning as in Figs. 24 and 25.

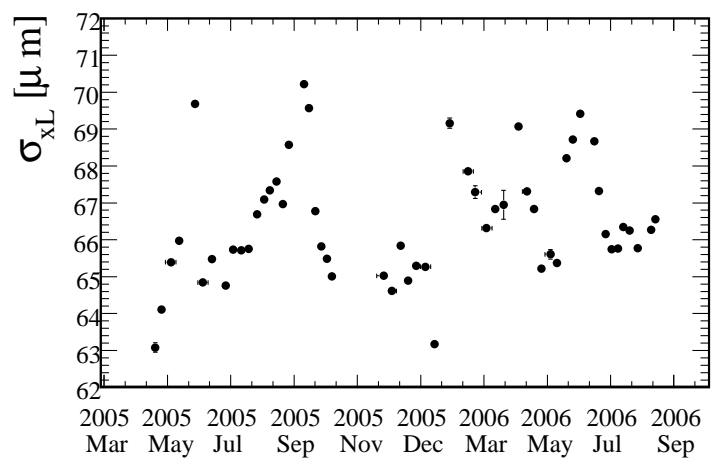

Figure 27. Medium-term history of the horizontal luminous size $\sigma_{x \mathcal{L}}$, as measured using the DOCA distribution. The errors are statistical only (and in many cases smaller than the data point).

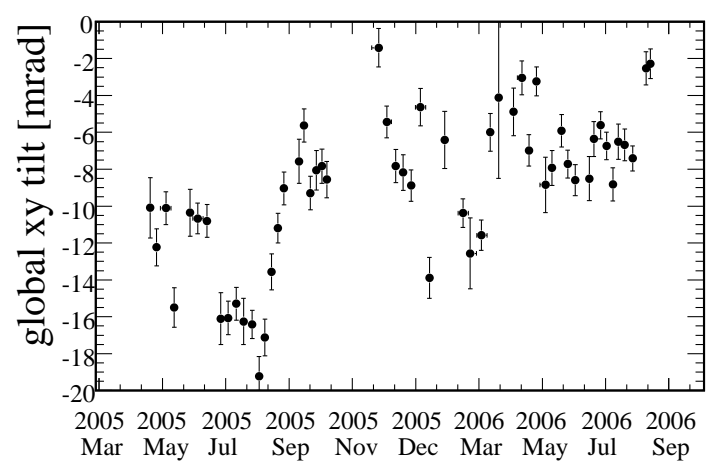

Figure 28. Medium-term history of $t_{x y}$, the azimuthal tilt of the luminous ellipsoid, as measured using the DOCA distribution. The errors are statistical only. 


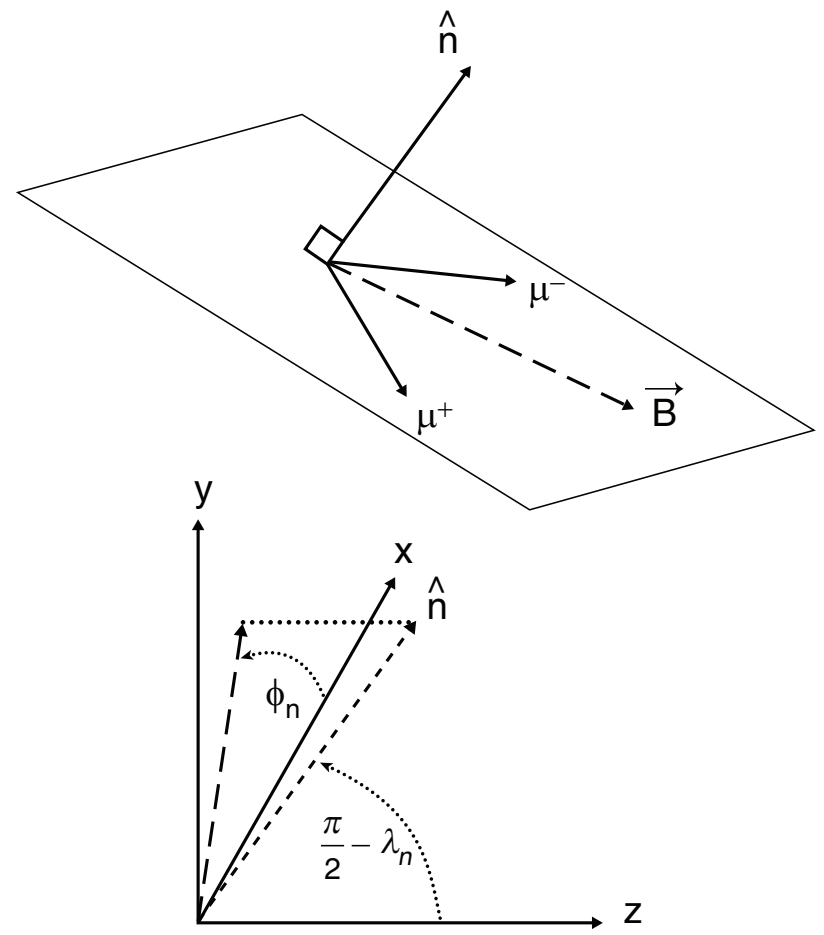

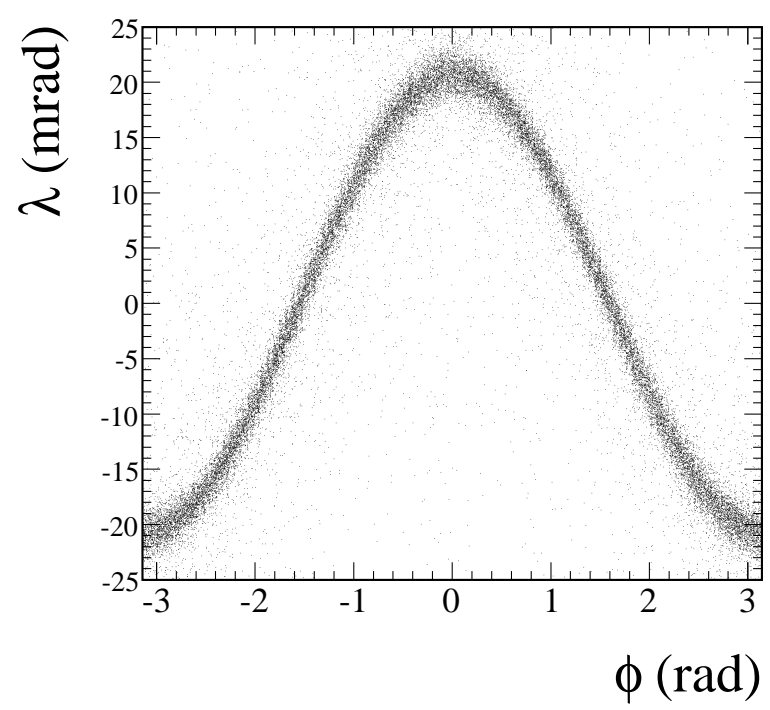

Figure 30. Measured $\mu$-pair distribution in the $\lambda_{n}-\phi_{n}$ plane, in the BABAR reference frame. The nominal $20 \mathrm{mrad}$ horizontal angle between the PEP-II collision axis and the detector coordinate system is clearly visible. plement of its polar angle) and by its azimuthal angle $\phi_{n}$, the angle its $x-y$ projection makes with the horizontal axis $x$ (bottom). 


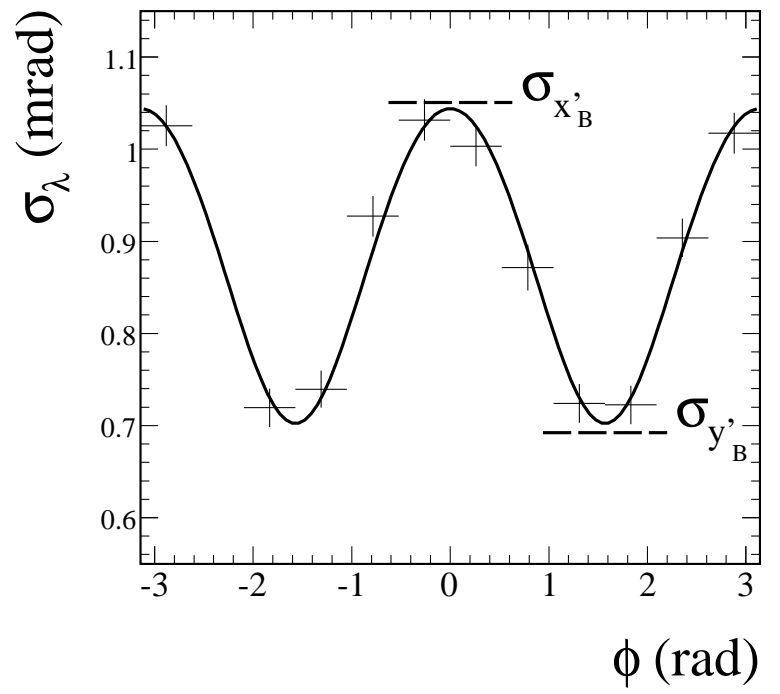

Figure 31. Azimuthal dependence of the angular spread $\sigma_{\lambda_{n}}$, corrected for initial-state radiation and detector resolution. A boost angular spread of $\sigma_{x_{\mathrm{B}}^{\prime}}=1.05$ and $\sigma_{y_{\mathrm{B}}^{\prime}}=0.69 \mathrm{mrad}$ are measured in this sample.

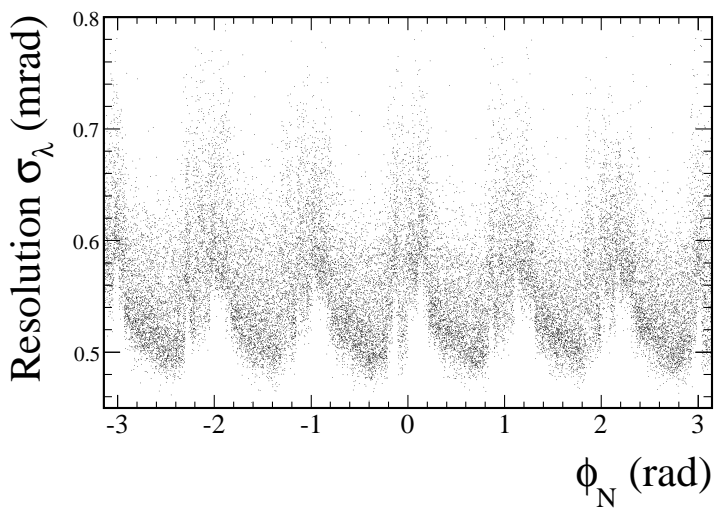

Figure 32. Azimuthal dependence of the detector resolution in dip angle $\lambda_{n}$. Although most of the dilution is caused by the coarser tracking resolution of the BABAR drift chamber, the six-fold symmetry of the silicon vertex tracker is apparent.
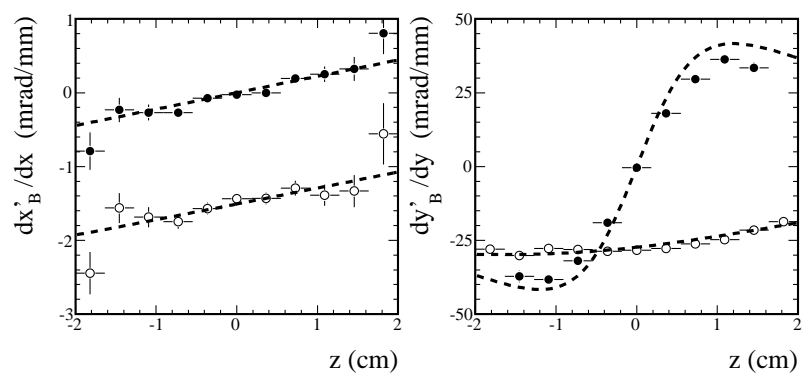

Figure 33. Longitudinal dependence of the $x$ $\bar{x}_{\mathrm{B}}$ (left) and $y-\bar{y}_{\mathrm{B}}^{\prime}$ (right) correlation in Monte Carlo simulation, without (closed circles) and with (open circles) the distortion caused by detector resolution. The dashed curves show the $z$-dependence predicted by Eq. 16 . 


\subsubsection{Signatures of the Vertical Hourglass Effect}

The longitudinal dependence of the verticalboost angular spread is given by (see Sec. 3.5):

$$
\begin{aligned}
& \sigma_{y_{\mathrm{B}}^{\prime}}^{2}= \\
& \left\{\left(\epsilon_{y-} \beta_{y-}^{*}+\epsilon_{y+} \beta_{y+}^{*}\right)\left(f_{-}^{2} \frac{\epsilon_{y-}}{\beta_{y-}^{*}}+f_{+}^{2} \frac{\epsilon_{y+}}{\beta_{y+}^{*}}\right)\right. \\
& \left.\quad+\frac{\epsilon_{y-}}{\beta_{y-}^{*}} \frac{\epsilon_{y+}}{\beta_{y+}^{*}}\left(f_{-} \alpha_{y+} \beta_{y+}^{*}-f_{+} \alpha_{y-} \beta_{y-}^{*}\right)^{2}\right\} \\
& \quad /\left[\epsilon_{y-} \beta_{y-}^{*}\left(1+\alpha_{y-}^{2}\right)+\epsilon_{y+} \beta_{y+}^{*}\left(1+\alpha_{y+}^{2}\right)\right]
\end{aligned}
$$

The first line describes the boost distribution at the interaction point, or more precisely at the waist $\left(\alpha_{y}=0\right)$. There no correlation exists between the vertical angles of the incoming particles and the $y$-position of the collision: the boost angular spread is the incoherent sum of the energyweighted individual beam spreads. In contrast, far from the IP $\left(\left|\alpha_{y}\right| \gg 1\right)$ the second line dominates: the $y$-position of the $\mu^{+} \mu^{-}$vertex is completely correlated with the vertical angles of the colliding $e^{ \pm}$. In this regime, the angular spread of beam particles contributing to collisions is proportional to the vertical size of the luminous region divided by the longitudinal distance to the IP. Each collision is "head-on", but the direction of the effective collision axis varies as a function of $y$ : it always points back to the nominal IP.

Figure 34 (left) illustrates the measurement of the vertical IP $\beta$-function, using simulated muon pairs generated with three different values of $\beta_{y-}^{*}=\beta_{y+}^{*}$, and reflecting representative event statistics. The boost distribution is constructed from a wider angular range of colliding particles at the waist than it is at large $|z|: \sigma_{y_{\mathrm{B}}^{\prime}}$ measurements show excellent sensitivity to changes in the common value of $\beta_{y}^{*}$.

The distance scale over which the $y-y^{\prime}$ correlation evolves is also $\beta_{y}^{*}$. Experimentally, this correlation can be probed directly by measuring the $y$-dependence of the mean vertical boost angle $\overline{y^{\prime}}{ }_{\mathrm{B}}$ (Fig. 34, right). Eq. 16 describes the expected relation between the measured observables and the actual beam parameters. Detector-resolution effects, which are large compared to the vertical luminous size, degrade the effectiveness of the method, but some constraining power remains.
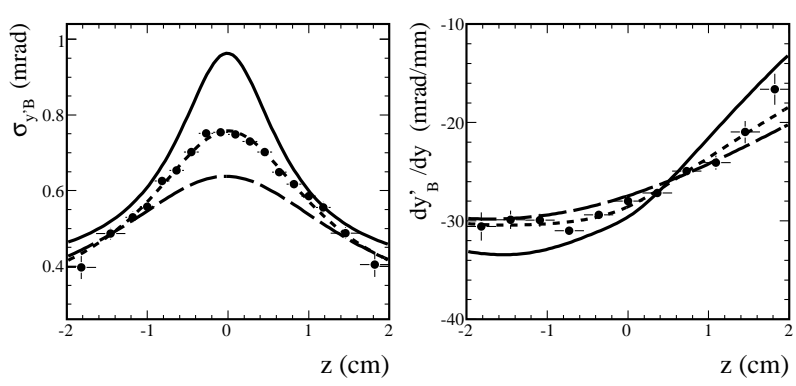

Figure 34. Impact of $\beta_{y}^{*}$ on the $z$-dependence of the vertical-boost angular spread $\sigma_{y_{\mathrm{B}}^{\prime}}$ (left), and of the position-angle correlation (right), predicted using a full detector simulation. The curves are fits to simulated data for $\beta_{y}^{*}=14$ (long dashes), 10 (short dashes) and $6 \mathrm{~mm}$ (solid line). The points with error bars are shown for the $10 \mathrm{~mm}$ simulation to indicate the sensitivity to $\beta_{y}^{*}$ variations. The muon pairs are generated assuming $\epsilon_{y-}\left(\epsilon_{y+}\right)=2.3(1.4) \mathrm{nm}-\mathrm{rad}$ and $z_{y-}^{w}=z_{y+}^{w}=0$, and neglecting beam-beam effects.

Figure 35 shows the two-dimensional $\chi^{2}$ contours for a fit of $\epsilon_{y-}, \epsilon_{y+}$, and $\beta_{y, B}^{*}\left(=\beta_{y+}^{*}\right.$ $=\beta_{y^{-}}^{*}$ ) to a measurement of $\sigma_{y_{\mathrm{B}}^{\prime}}$ using simulated data. In this fit, the emittances are poorly determined and highly correlated (left), and the $\beta_{y}^{*-}$ $\epsilon_{y-}$ plot (right) also exhibits structure (albeit to a lesser degree). This is because the data allow a precise determination of the peak value of $\sigma_{y_{\mathrm{B}}^{\prime}}$ (Fig. 34, left), but do not constrain the large$|z|$ tails for lack of statistics. More information is thus needed to separate the $e^{+}$and $e^{-}$emittances. The $y-\bar{y}^{\prime}{ }_{\mathrm{B}}$ correlation provides this additional constraint: Figure 36 shows the $\chi^{2}$ contours of the same parameters in the combined fit to simulated, simultaneous measurements of $\sigma_{y_{\mathrm{B}}^{\prime}}$ and $\delta{\overline{y^{\prime}}}_{\mathrm{B}}(z) / \delta y$. The fit reproduces the true (generated) value of $\beta_{y}^{*}$ to within $0.3 \mathrm{~mm}$, with a statistical accuracy of $\pm 0.3 \mathrm{~mm}$ for a simulated exposure of $4 \mathrm{fb}^{-1}$.

Fig. 37 displays the sensitivity of boost observables to the longitudinal position of the vertical waists, using a simplified simulation ("toy Monte Carlo"). The longitudinal position of the 

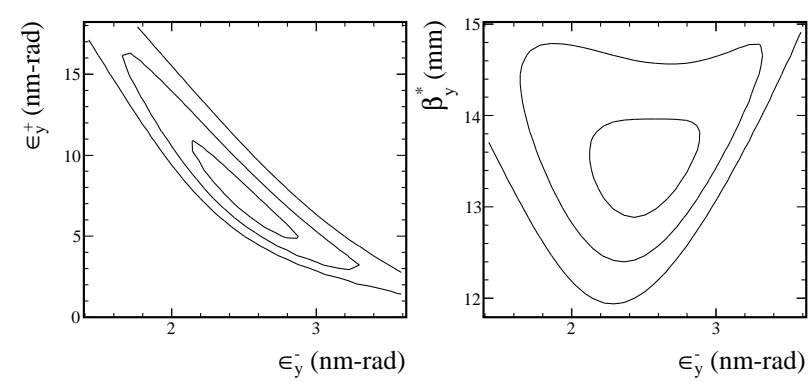

Figure 35. 1-, 2-, and 3- $\sigma$ contours for the vertical emittances and (assumed common) vertical IP $\beta$-function $\beta_{y, B}^{*}$ extracted from a fit to $\sigma_{y_{\mathrm{B}}^{\prime}}$ in a Monte Carlo simulation that includes the full detector response. The muon pairs are generated assuming $\epsilon_{y-}\left(\epsilon_{y+}\right)=2.3(1.4) \mathrm{nm}-\mathrm{rad}$ and $\beta_{y-}^{*}=\beta_{y+}^{*}=12.1 \mathrm{~mm}$, and neglecting beambeam effects.
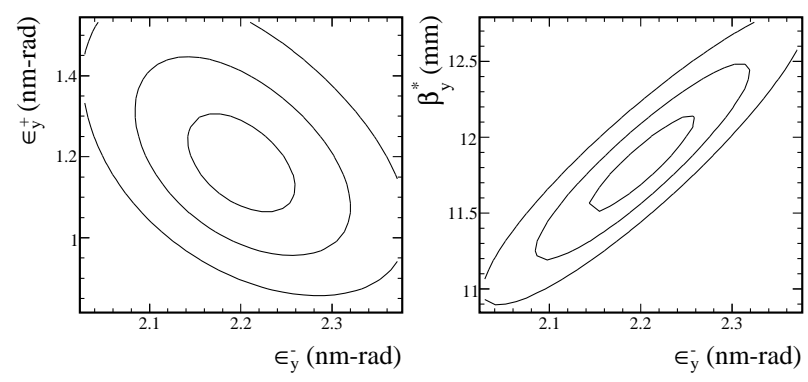

Figure 36. 1-, 2-, and 3- $\sigma$ contours for the vertical emittances and IP $\beta$-function $\beta_{y, B}^{*}$ extracted from a combined fit to $\sigma_{y_{\mathrm{B}}^{\prime}}$ and the $y$ - $\bar{y}_{\mathrm{B}}{ }_{\mathrm{B}}$ correlation, in a Monte Carlo simulation that includes the full detector response. The muon pairs are generated assuming $\epsilon_{y-}\left(\epsilon_{y+}\right)=2.3$ (1.4) nm-rad and $\beta_{y-}^{*}=\beta_{y+}^{*}=12.1 \mathrm{~mm}$, and neglecting beambeam effects.

peak angular divergence trails the waist of the higher-energy electron beam. Separating the electron and positron waists distorts the shape of the $\delta \bar{y}^{\prime}{ }_{\mathrm{B}}(z) / \delta y$ measurement, rather than simply shifting the whole curve towards the waist of one beam or the other. This simplified Monte Carlo lacks the ability to model correlated detector errors (which are however included in the full detector simulation used for Fig. 34).
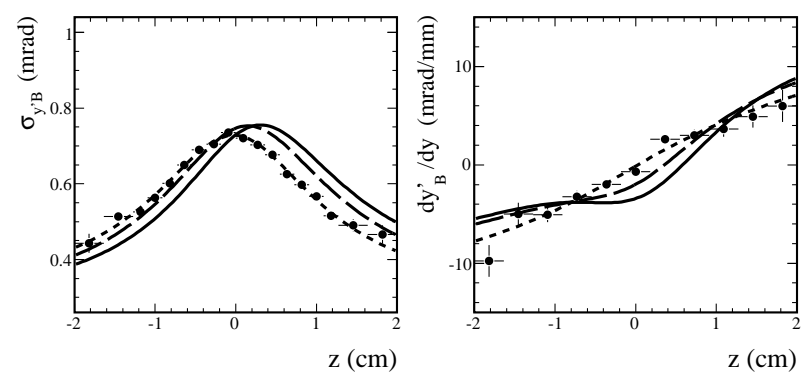

Figure 37. Impact of the waist positions on the $z$-dependence of the vertical-boost angular spread (left) and of the position-angle correlation (right), predicted using a parameterized simulation. The curves are fits to simulated data for $z_{y-}^{w}=z_{y+}^{w}=0$ (short dashes); $z_{y-}^{w}=+2 \mathrm{~mm}$, $z_{y+}^{w}=-2 \mathrm{~mm}$ (long dashes); and $z_{y-}^{w}=+4 \mathrm{~mm}$, $z_{y+}^{w}=-4 \mathrm{~mm}$ (solid curve). The points with error bars are shown for the $\left\{z_{y-}^{w}=z_{y+}^{w}=0\right\}$ simulation to indicate the sensitivity to waist separation. The muon pairs are generated assuming $\epsilon_{y-}\left(\epsilon_{y+}\right)=2.3(1.4) \mathrm{nm}$-rad and $\beta_{y-}^{*}=\beta_{y+}^{*}=$ $10 \mathrm{~mm}$, and neglecting beam-beam effects.

\subsubsection{Predicted Beam-beam Signatures}

Beam-beam-induced distortions of the $e^{ \pm}$ phase space at the PEP-II IP are known to induce a significant reduction of the predicted specific luminosity with increasing bunch currents; these dynamic changes are reflected in the evolution of the three-dimensional luminosity distribution. But beam-beam forces are also expected to produce measurable effects in the angular distribution of the boost vector.

The spread in vertical boost angle predicted by the beam-beam simulation of Ref. [27] is shown in Fig. 38 for very low and for nominal $e^{ \pm}$bunch currents: both the peak value of $\sigma_{y_{\mathrm{B}}^{\prime}}$ and the shape 
of its $z$-dependence are impacted. The analysis of the evolution of the individual beam distributions indicates that the peak angular spread grows mainly due to a sizable increase in the vertical emittance of the beams; the associated, albeit moderate reduction in $\beta_{y}^{*}$ accounts for the slightly sharper fall-off, at nominal bunch currents, of the $z$-dependence.

The effects of the beam-beam interaction upon the $y-\bar{y}_{\mathrm{B}}^{\prime}$ correlation are illustrated in Fig. 39. The predictions of Eq. 16 (which ignores beambeam distortions) are in good agreement with the simulation at low current; at nominal currents the general features are well predicted, but the quantitative agreement is not as good because the bunches now deviate significantly from their original Gaussian shape, and the effective waist position is slightly shifted by the vertical pinch effect [36]. Note, however, that the beam-beam induced distortions illustrated here have a much weaker impact than, and will be diluted by, the detector resolution effects shown in Fig. 33.
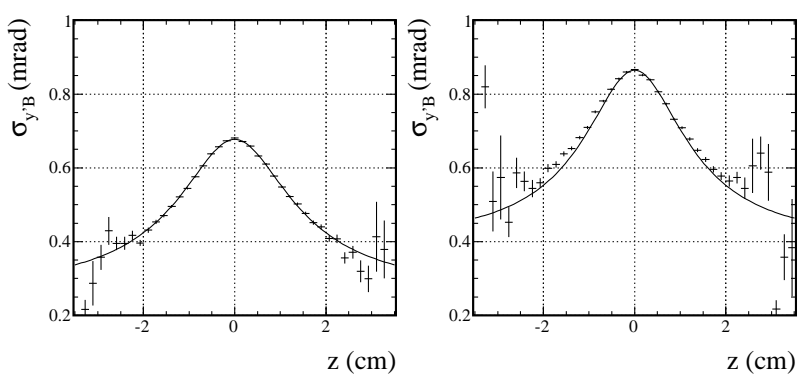

Figure 38. $z$-dependence of the vertical-boost angular spread, predicted by the beam-beam simulation at $e^{+}$and $e^{-}$bunch currents of $0.010 \times$ $0.006 \mathrm{~mA}^{2}$ (left) and $1.60 \times 0.92 \mathrm{~mA}^{2}$ (right). The points with statistical error bars are the simulated angular spread in each $z$ bin. The solid curve is from Eq. 26 using emittances and $\beta$-functions inferred from the simulated $e^{ \pm}$distributions at the IP.
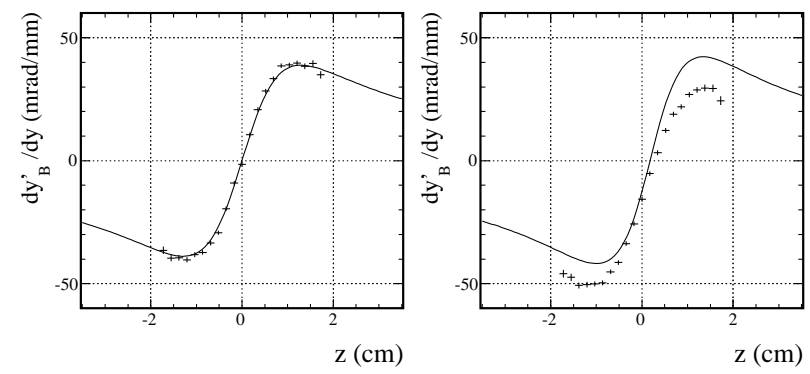

Figure 39. $z$-dependence of the verticalboost angle-position correlation, predicted by the beam-beam simulation at very low (left) and at nominal (right) bunch currents, and assuming perfect detector resolution. The points with statistical error bars are the simulated angularposition correlation in each $z$ bin. The solid curve is from Eq. 16 using emittances and $\beta$-functions inferred from the simulated $e^{ \pm}$distributions at the IP.

\subsubsection{History of Vertical Phase-Space Pa- rameters}

PEP-II has been operating for over eight years, allowing to follow the evolution of the beam parameters extracted from our fits, and to produce history plots that reflect changes in the machine optics. To perform this study, the data was partitioned into intervals approximately two weeks long, each containing a sufficient number of $e^{+} e^{-} \rightarrow \mu^{+} \mu^{-}$events to perform an adequately precise measurement of $\beta_{y, B}^{*}$. For each of these intervals, the measured $z$-dependence of $\sigma_{y_{\mathrm{B}}^{\prime}}$ and of the $y-\bar{y}^{\prime}{ }_{\mathrm{B}}$ correlation was fit for the beam parameters. Figures 40 and 41 show typical fits over one such interval. The vertical angular spread at $z=0$ (Fig. 40, right) significantly exceeds the single-beam estimates of Table 1 , as expected in the presence of the beam-beam effects discussed above. The $y-\bar{y}_{\mathrm{B}}{ }_{\mathrm{B}}$ correlation measurement exhibits (Fig. 41, right) the strong dilution expected from the Monte Carlo simulation (see Fig. 33, right), yet provides an effective constraint in the combined fit for the beam parameters. The typical statistical error on such a fit amounts to 0.7 
$\mathrm{mm}$ on $\beta_{y, B}^{*}$. The corresponding systematic uncertainties add up to approximately $\pm 1 \mathrm{~mm}$.
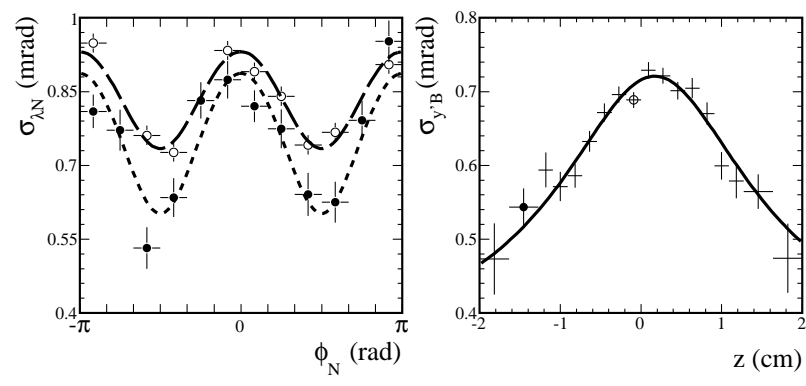

Figure 40. Measurement of the longitudinal dependence of the vertical-boost angular spread. Left: azimuthal dependence of the dip angle of the decay plane, at $z \approx 0$ (open circles) and for $z \approx-1.5 \mathrm{~cm}$ from the IP (full circles). The corresponding dashed lines represent the fits that yield $\sigma_{x_{\mathrm{B}}^{\prime}}$ and $\sigma_{y_{\mathrm{B}}^{\prime}}$ at those z positions. Right: measured $z$-dependence of $\sigma_{y_{\mathrm{B}}^{\prime}}$. The solid line is a fit using the parameterization of Eq. 26. The two highlighted data points (full and open circles) correspond to the two fits shown on the left.

The time evolution of $\beta_{y}^{*}$, as measured by the boost method and assuming $\beta_{y+}^{*}=\beta_{y-}^{*}=\beta_{y, B}^{*}$, is presented in Fig. 42, and compared with the $\beta_{y \text {,eff }}^{*}$ measurements extracted from the longitudinal luminosity distribution (Sec. 5.1.3). The two methods yield consistent results, confirming the apparent discrepancy with the low-current phaseadvance measurements.

Fig. 43 shows the combined fit results for the $e^{ \pm}$emittances. As evidenced by the size of the error bars, the fit is much more sensitive to the electron than to the positron beam emittance, because of the higher HER beam energy (see also Fig. 36, left). A systematic difference (not shown) between single and combined fits remains to be understood. These vertical phase-space measurements will be confronted with constraints from the luminous-size analysis in Sec. 9.3.
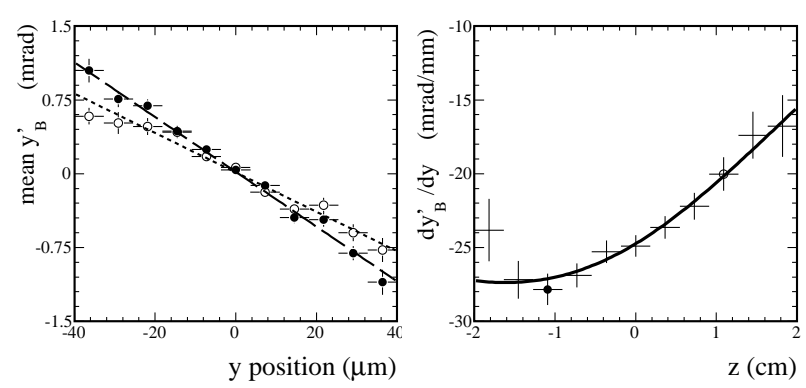

Figure 41. Left: correlation between vertical vertex position and mean boost angle, for $\mu^{+} \mu^{-}$vertices in the intervals $-1.3<z<-0.9 \mathrm{~cm}$ (full circles) and $0.9<z<1.3 \mathrm{~cm}$ (open circles). The slopes of the corresponding straight-line fits (left, dashed lines) are plotted as a function of $z$ in the right figure, and their longitudinal dependence fit (solid line) using the parameterization of Eq. 16. The two highlighted data points (open and closed circle) in the right figure, correspond to the two fits shown on the left.

\subsection{Characterization of Horizontal Phase Space}

A similar set of boost-vector measurements can be performed in the horizontal plane. Because the longitudinal evolution of horizontal observables is determined by $\beta_{x}^{*}$, which is considerably larger than the bunch lengths, horizontal-hourglass effects remain undetectable. The horizontal-boost analysis therefore provides weaker constraints on the beam parameters than the corresponding vertical measurements of Sec. 7.2. Some $z$-averaged measurements, however, remain useful: for instance, because of the $e^{+}-e^{-}$energy asymmetry, the horizontal boost angular spread is largely dominated by two parameters, the horizontal emittance and IP $\beta$-function of the electron beam (Eq. 13). The online measurement of $\sigma_{x^{\prime}}$ relies on this approximation.

\subsubsection{Beam-beam Signatures}

The effect of the beam-beam interaction on horizontal-boost observables was studied using the same simulations as in Sec. 7.2.2. The pre- 

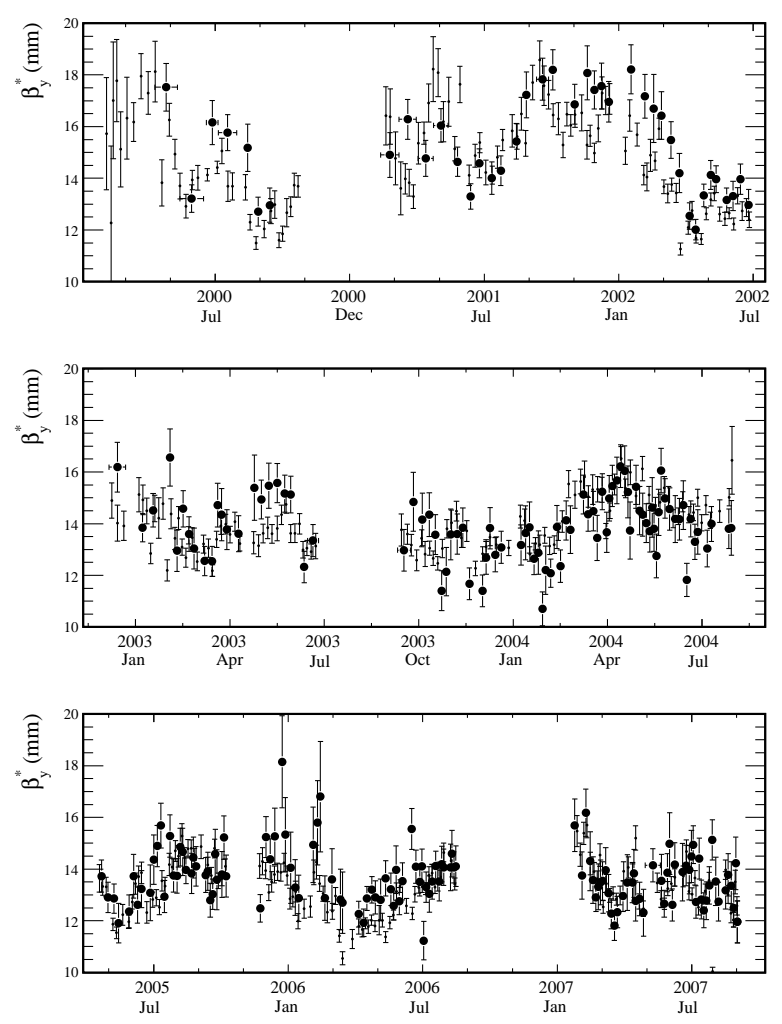

Figure 42. Six-year history of $\beta_{y}^{*}$ measured by the boost method (circles) and extracted from the fit to the longitudinal luminosity profile (points).

dicted spread in horizontal boost angle is shown in Fig. 44 for very low and for nominal $e^{ \pm}$bunch currents. The beam-beam interaction induces a $\sim 50 \%$ increase in horizontal angular spread, accompanied by a sizable reduction in horizontal luminous size (Fig. 45). These predictions can be directly confronted with $B A B A R$ measurements under typical high-luminosity conditions.

The $z$-dependence of the horizontal-boost angular spread is shown in Fig. 46 (left). As expected from the beam-beam simulation, the observed angular spread is significantly larger than that naively computed from single-beam parameters (Table 1). The positive slope of its $z$ dependence suggests that the horizontal waist
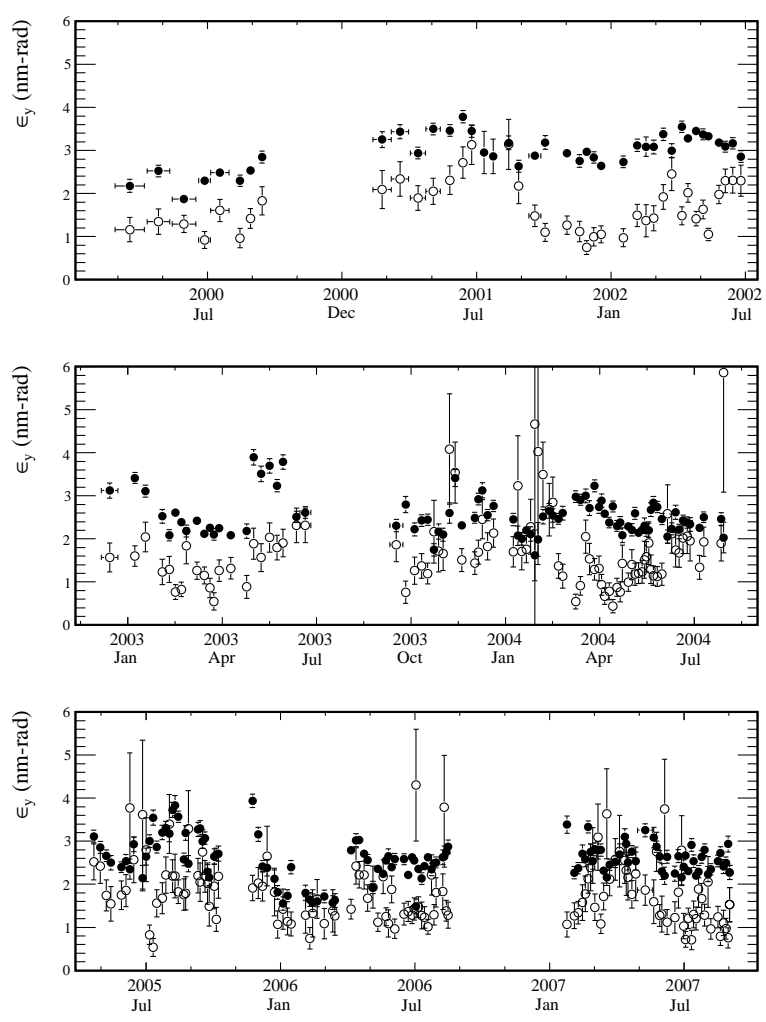

Figure 43. Six-year history of the $e^{-}$(closed circles) and $e^{+}$(open circles) vertical emittances, from the combined fit to $\sigma_{y_{\mathrm{B}}^{\prime}}(z)$ and $\delta{\overline{y^{\prime}}}_{\mathrm{B}}(z) / \delta y$.

may be offset in the positive direction by a considerable fraction of $\beta_{x}^{*}$. This interpretation is suggested by analogy with the vertical angular spread (Fig. 40, right): a positive slope is observed at negative $z$ (with respect to the waist), and vice versa. The horizontal measurement samples only a small fraction of the hourglass shape, compared to the vertical measurement, before "running out" of luminosity at large $|z|$.

Figure 46 (right) shows the $z$-dependence of the horizontal luminous size $\sigma_{x \mathcal{L}}$, measured using a simple vertexing technique and resolution correction (Sec. 4.2.2). The reported magnitude is comparable to that expected in the presence of strong beam-beam effects (Fig. 45, right). It also is fully 

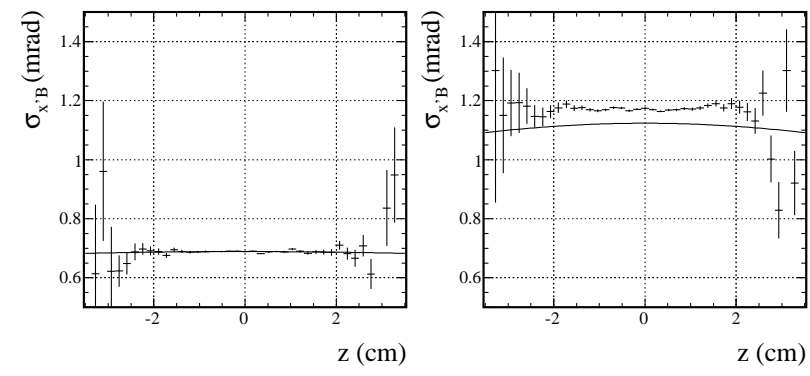

Figure 44. Longitudinal dependence of the horizontal-boost angular spread, predicted by the beam-beam simulation at very low (left) and at nominal (right) bunch currents (see Fig. 38). The points with statistical error bars are the simulated angular spread in each $z$ bin. The solid curves are from Eq. 14 (with $x$ substituted for $y$ ) using horizontal emittances and $\beta$-functions inferred from Gaussian fits to the simulated $e^{ \pm}$distributions at the IP.
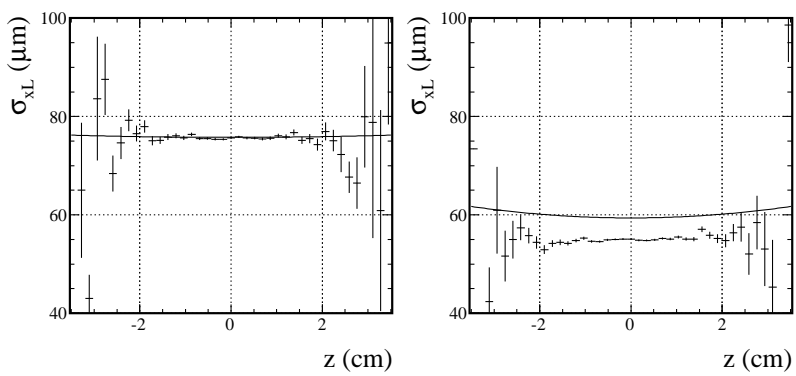

Figure 45. As in Fig. 44, but for the horizontal luminous size, at very low (left) and at nominal (right) bunch currents. The points with error bars are the values of $\sigma_{x \mathcal{L}}$ predicted by the simulation in each $z$ bin. The solid curves reflect the combination of Eqs. 8 and 9, using horizontal emittances and $\beta$-functions inferred from the simulated $e^{ \pm}$distributions.

consistent, in spite of very different systematics, with the horizontal luminous size measured dur- ing the same period by the much more sophisticated impact-parameter method (Fig. 27). However, the slight $z$-dependence of $\sigma_{x \mathcal{L}}$ suggests a horizontal-waist shift of opposite sign to that implied by Fig. 46 (left). A more extensive study of the impact of beam-beam distortions on the shape of the luminous ellipsoid and on the boost distributions, would be required to lift this apparent (but minor) contradiction.

The five-year history of the horizontal luminous size and $e^{-}$angular divergence (which dominates the horizontal-boost angular spread), reconstructed from archived BABAR data, is presented in Fig. 47. The sharp reduction in $\sigma_{x \mathcal{L}}$ and correlated increase in $\sigma_{x^{\prime}}$ - were accompanied by an appreciable luminosity increase; these step changes reflect the onset of the significant dynamic- $\beta$ enhancement associated with the move to half-integer tunes.
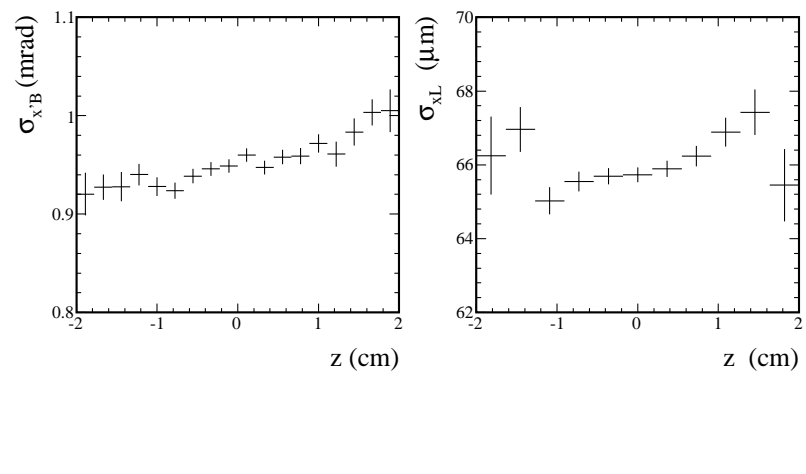

Figure 46. Measurement of the longitudinal dependence of the horizontal boost angular spread (left) and of the horizontal luminous size (right).

\subsubsection{Crossing Angles}

For head-on collisions, the average boost direction is aligned with the longitudinal axis of the luminous ellipsoid. When a crossing angle is introduced, the difference between the mean boost angle and the luminous tilt (Sec. 3.3) provides a measure of the actual crossing angle, as described in Eq. 12. The relation depends upon the relative difference in transverse beam sizes at the 

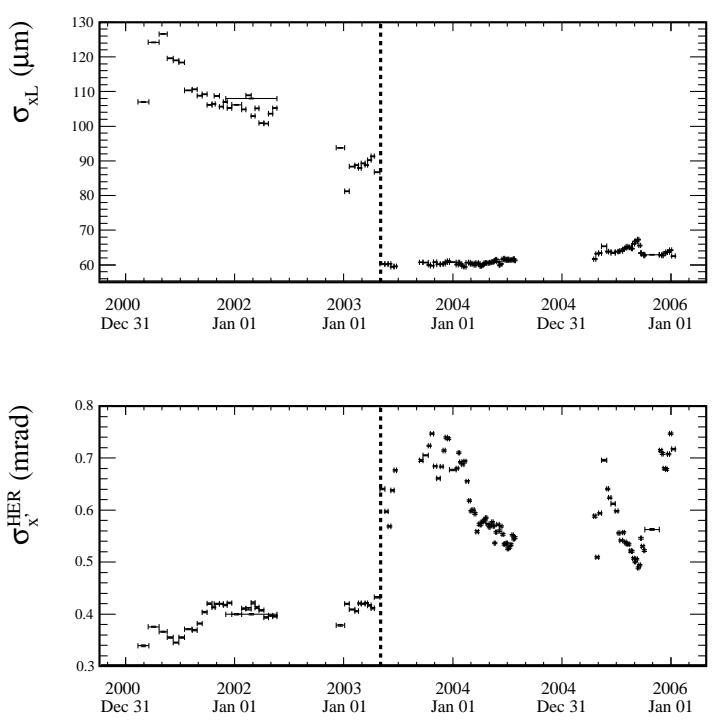

Figure 47. Long-term evolution of $\sigma_{x \mathcal{L}}$ (top) and $\sigma_{x_{-}^{\prime}}$ (bottom). The dotted vertical line marks the date when the horizontal tunes of both rings were moved very close to the half-integer.

interaction point, which for typical beam sizes introduces but a small fractional correction to the naive horizontal crossing-angle estimate.

Figure 48 (left) displays the history of the measured mean horizontal boost angle $\overline{x^{\prime}}{ }_{B}$ and horizontal luminous tilt $\overline{x^{\prime}} \mathcal{L}$. Both observables display large, correlated steps, corresponding either to reestablishing accelerator operation after major maintenance periods or to re-optimizing beam conditions. In contrast, the resulting 0.3 - $0.4 \mathrm{mrad}$ crossing angle (Fig. 48 , right) exhibits much smaller variations. Its non-zero average is consistent with the optimum crossingangle recorded by beam-orbit monitors during dedicated beam-beam experiments: the best luminosity results from an empirical compromise between crossing-angle and parasitic-crossinginduced beam separation [34].

In the vertical, the much smaller beam size leads to considerably tighter crossing-angle tol- erances, not only to ensure the geometrical overlap of the ribbon-like beams, but mainly because the deleterious impact of the beam-beam interaction rapidly grows when the vertical crossing angle exceeds $\sim 10 \mu \mathrm{rad}$; a similar sensitivity has been observed in KEKB [35]. Figure 49 displays the measured mean vertical boost angle $\overline{y^{\prime}}$ B and the vertical luminous tilt $\overline{y^{\prime}} \mathcal{L}$, whose difference is consistent with zero considering the measurement uncertainties. The BABAR detector reports measurements of these angles every five minutes with typical resolutions of 0.1 and $0.2 \mathrm{mrad}$ respectively.
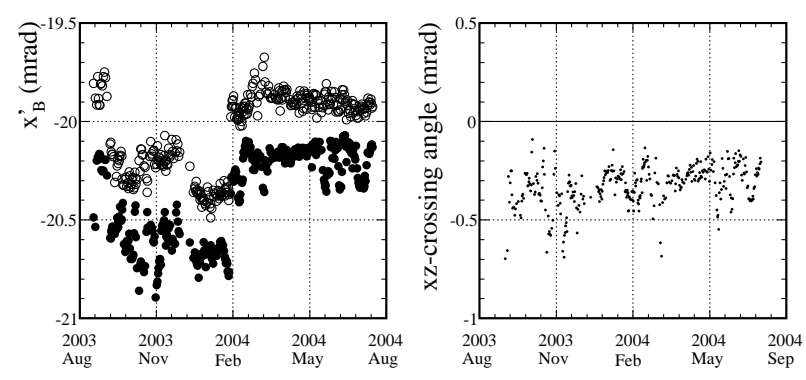

Figure 48. Left: mean horizontal boost $\overline{x^{\prime}}$ B (closed circles), compared to the horizontal tilt of the luminous ellipsoid (open circles). Their difference (right) measures the horizontal crossing angle.

\section{Stored-beam profile measurements}

\subsection{Methodology}

The lattice functions that relate the beam profile measured at a synchrotron-light monitor to the emittances of the two eigenmodes (Eqs. 17,18) are measured by resonant excitation and analyzed in the context of the MIA formalism (Sec. 3.6). The extracted eigenemittances can then be combined with these same lattice functions at the IP to yield the transverse parameters of each beam at that particular location.

At any point around the ring, the lattice functions above can be converted into, or alternatively 

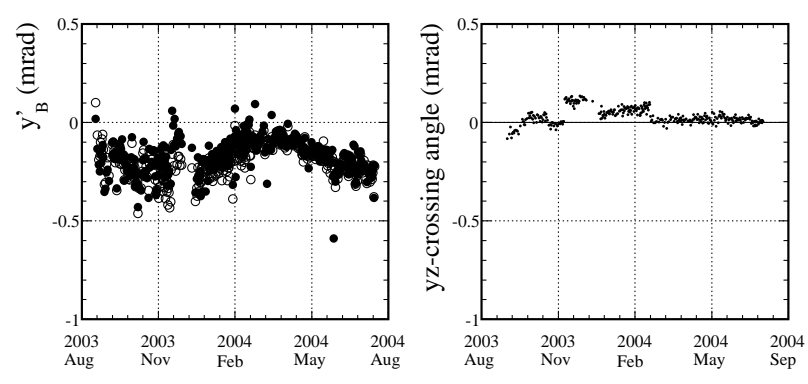

Figure 49. Left: mean vertical boost ${\overline{y^{\prime}}}_{\mathrm{B}}$ (closed circles), compared to the vertical tilt of the luminous ellipsoid (open circles). Their difference (right) measures the vertical crossing angle. The steps in the measured crossing angle coincide with down-times indicating a possible dependence upon detector alignment changes.

expressed in terms of, the fully coupled one-turn matrix $\mathbf{M}$ at that location [37]. Such one-turn matrices are therefore required at three locations in the LER: those at the SXML and SLML profile monitors are used to extract the eigenemittances and the beam tilt-angle from the measured beam profiles, while the one-turn matrix at the IP is needed to convert the results back into IP beam sizes. In practice, these one-turn matrices $\mathbf{M}$ are built from consecutive transfer matrices $\mathbf{T}$ (which are themselves based on the MIA data): one from the IP to the SXML, a second one from the SXML to the SLML, and finally a third matrix connecting the SLML back to the LER IP. The procedure is similar in the HER, except that here only two one-turn matrices are required, one at the SLMH and one at the IP.

The above procedure is well established and has been routinely used in numerous storage rings (at least in a simplified form applicable to the nocoupling limit). As described above however, this analysis is valid only to the extent that the beambeam interaction does not significantly alter the lattice functions in either ring. Its application to PEP-II, even at moderate bunch currents, requires to take carefully into account the mutual focusing of the two beams.

\subsection{Transverse Profile Analysis in the Presence of Beam-Beam Effects}

The effect of the beam-beam interaction on the one-turn matrices is modeled in the linear approximation, by inserting a matrix $\mathbf{B}_{ \pm}$at the IP, with off-diagonal focusing terms that depend on the charge and transverse sizes of the other beam. For example, the beam-beam matrix $\mathbf{B}_{+}$acting on the positron beam is given by:

$$
\left(\begin{array}{cccc}
1 & 0 & 0 & 0 \\
\frac{-2 r_{e} N_{-}}{\gamma+\sigma_{x-}\left(\sigma_{\left.x-+\sigma_{y-}\right)}\right.} & 1 & 0 & 0 \\
0 & 0 & 1 & 0 \\
0 & 0 & \frac{-2 r_{e} N_{-}}{\gamma+\sigma_{y-}\left(\sigma_{x-}+\sigma_{y-}\right)} & 1
\end{array}\right),
$$

where $r_{e}$ is the classical electron radius, $N_{-}$is the number of electrons per bunch (or per slice, see below), $\gamma_{+}$is the relativistic factor of the positrons, and $\sigma_{x-}\left(\sigma_{y-}\right)$ is the horizontal (vertical) size of the $e^{-}$beam at the place where the collision occurs. Because one is interested in solving for the lattice parameters at the IP itself, the beam-beam matrix is split into two parts, inserting on either side of the IP the matrix $\sqrt{\mathbf{B}_{+}}$(simply obtained by omitting the 2 in the off-diagonal terms). In the case of the HER for instance, the one-turn matrix at the IP is given by

$$
\mathbf{M}_{\mathrm{IP}}=\sqrt{\mathbf{B}_{-}} \mathbf{T}_{\mathrm{SLMH} \rightarrow \mathrm{IP}} \mathbf{T}_{\mathrm{IP} \rightarrow \mathrm{SLMH}} \sqrt{\mathbf{B}_{-}} .
$$

To take into account the longitudinal spread of the beams and their hourglass shape, the $e^{ \pm}$ longitudinal particle distributions, assumed to be Gaussians, are divided into slices of equal charge; each slice collides sequentially with all the slices of the other beam. When building the corresponding $\mathbf{B}_{ \pm}$matrices, the charge of each slice is assumed to be concentrated at the longitudinal centroid of that slice, with a transverse distribution that depends, at each step of the collision process, on the distance of the slice to the (vertical) waist. For a given beam, all slices have the same transverse emittance, but each slice is associated with its own one-turn matrix at the IP (and at each light monitor); the two eigenemittances are determined from the measured beam profiles and the slice-averages of the coefficients of the emittances in Eqs. 17 and 18. This procedure thus takes into account the longitudinal 
Table 5

Beam parameters at the PEP-II IP extracted from recent profile-monitor measurements at moderate beam currents.

\begin{tabular}{|c|c|c|c|c|}
\hline Parameter & \multicolumn{2}{|c|}{ LER $\left(e^{+}\right)$} & \multicolumn{2}{|c|}{ HER $\left(e^{-}\right)$} \\
\hline "Beam current $(\mathrm{mA})$ & \multicolumn{2}{|c|}{ 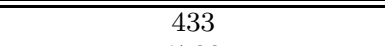 } & \multicolumn{2}{|c|}{124} \\
\hline Number of bunches & \multicolumn{2}{|c|}{1722} & \multicolumn{2}{|c|}{1722} \\
\hline 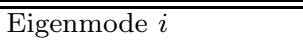 & $\overline{c i=1(x \text {-like })}$ & $\overline{i=2(y \text {-like })}$ & $\overline{c i=1(x \text {-like })}$ & $\overline{c i=2(y \text {-like })}$ \\
\hline \multicolumn{5}{|c|}{ Single-beam analysis, neglecting the beam-beam interaction } \\
\hline$\overline{\epsilon_{i}(\mathrm{~nm}-\mathrm{rad})}$ & 28.3 & 2.3 & 111 & 1.1 \\
\hline$\beta_{i}^{*}(\mathrm{~mm})$ & 259 & 7.9 & 419 & 10.6 \\
\hline$\sigma_{i}(\mu \mathrm{m})$ & 85 & 6.5 & 214 & 5.8 \\
\hline Tilt angle (degrees) & \multicolumn{2}{|c|}{0.71} & \multicolumn{2}{|c|}{-1.20} \\
\hline \multicolumn{5}{|c|}{ Beam-beam interaction accounted for by the iterative procedure of Sec. 8.2} \\
\hline$\epsilon_{i}(\mathrm{~nm}-\mathrm{rad})$ & 27.7 & 2.2 & 103 & 1.1 \\
\hline$\beta_{i}^{*}(\mathrm{~mm})$ & 248 & 7.7 & 228 & 10.2 \\
\hline$\sigma_{i}(\mu \mathrm{m})$ & 82 & 6.3 & 153 & 4.9 \\
\hline Tilt angle (degrees) & \multicolumn{2}{|c|}{0.64} & \multicolumn{2}{|c|}{-1.10} \\
\hline Beam-beam parameter & $2.5 \times 10^{-3}$ & $2.4 \times 10^{-3}$ & $9.7 \times 10^{-3}$ & $5.6 \times 10^{-3}$ \\
\hline
\end{tabular}

variation of the vertical beam-beam parameters during the collision process.

In general, the electron beam is tilted by an angle $\psi_{-}$at the location of each slice-slice collision, while the expression above for the beambeam force is in uncoupled coordinates. One must therefore apply a rotation matrix $\mathbf{R}_{-}$to the $\mathbf{B}_{+}$ matrix acting on the positron slice in order to get the rotated beam-beam matrix $\mathbf{B}_{\mathbf{r}+}$ :

$$
\mathbf{B}_{\mathbf{r}+}=\mathbf{R}_{-}^{-1} \mathbf{B}_{+} \mathbf{R}_{-},
$$

where

$$
\mathbf{R}_{-}=\left(\begin{array}{cccc}
\cos \psi_{-} & 0 & \sin \psi_{-} & 0 \\
0 & \cos \psi_{-} & 0 & \sin \psi_{-} \\
-\sin \psi_{-} & 0 & \cos \psi_{-} & 0 \\
0 & -\sin \psi_{-} & 0 & \cos \psi_{-}
\end{array}\right) .
$$

Equivalent considerations apply to the rotated matrix $\mathbf{B}_{\mathbf{r}-}$. The full sequence of rotated beambeam matrices then models the beam-beam interaction at the IP.

A difficulty with this technique is evident: the $e^{-}$beam size at the IP must be known in order to determine the $e^{+}$beam size there, and vice-versa. The solution is an iterative approach that starts with a zero beam-beam contribution and then gradually increases its strength. In the initial step the bunch populations $N_{\mp}$ are set to 0 , the ring lattices are independent, and the IP beam sizes and tilts can be estimated using the measured, unaltered lattice functions. These IP beam sizes are then used in the following iteration, $N_{\mp}$ set to a small non-zero value, the one-turn matrices altered to include the effect of the beam-beam kicks, and the eigenemittances computed anew. The procedure is repeated by progressively ramping up the charge $N_{\mp}$ in the opposing bunch used in $\mathbf{B}_{ \pm}$, until both bunch populations reflect the actual beam currents at the time the beam-profile measurements were recorded. The number of longitudinal slices depends on the strength of the beam-beam interaction in the data sample considered; it is chosen large enough for the iterative beam-beam correction to converge.

\subsection{Experimental results}

When the currents, and hence the beam-beam forces, are low enough, this procedure yields a physically plausible result, as illustrated in Table 5 . The profile monitor data were recorded in collision, immediately prior to a set of MIA lattice measurements. Figure 50 shows the IP beam parameters converging as the bunch populations are ramped up, in the analysis, from 0 (Table 5, center rows) to their actual value in 80 steps. The calculation then continues for a further 20 iterations with constant bunch charges, and all IP beam parameters (Table 5 , bottom rows) remain stable. A single longitudinal slice representing the entire bunch is sufficient to ensure conver- 
gence, and the results do not change when the number of slices is increased. The impact of the beam-beam interaction on the interpretation of the profile-monitor data is illustrated by the reduction of $\beta_{1}^{*}\left(e^{-}\right)$from 42 to $23 \mathrm{~cm}$, that reflects a significant onset of the dynamic- $\beta$ effect in the HER, as expected in view of the already sizable LER current.

At first sight, the transverse IP beam sizes appear reasonable: while in this experiment the luminosity was too low for directly measuring the luminous size using $B A B A R$, the inferred values of $\sigma_{y \mathcal{L}}$ and $\sigma_{x \mathcal{L}}$ (Table 6 ) are reasonably consistent with the range of measurements reported in Figs. 24 and 27, respectively. But closer scrutiny reveals inconsistencies. The product of convoluted beam sizes $\Sigma_{x} \times \Sigma_{y}$ inferred from profilemonitor data can be confronted with that directly measured by scanning one beam transversely across the other [11]-[12], as well as with that calculated from the measured specific luminosity $\mathcal{L}_{s p}$. After applying the required hourglass corrections [10] to the raw measurements of $\Sigma_{y}$ and $\mathcal{L}_{s p}$, the beam-beam scans and the specific luminosity yield values of $\Sigma_{x} \times \Sigma_{y}$ that are consistent within $10 \%$ (Table 6 ); in contrast, the result inferred using profile monitors is 60 $70 \%$ larger. Equivalently, the specific luminosity predicted using measured beam currents and synchrotron-light profiles would fall significantly short of that actually observed.

Similar measurements and other circumstantial evidence accumulated in the last few years of running suggests that the discrepancy is caused by the combination of at least two effects. First, the value of $\epsilon_{1}\left(e^{-}\right)$inferred from SLMH measurements (Table 5 ) is 1.5 to 2 times as large as that predicted by lattice simulations; this can be traced back to an overestimation of the horizontal beam size measured at the HER SLM, whose primary mirror is known to have suffered large thermal stresses that distort the image (the vertical measurement remains unaffected because it relies on the interferometer). Using as a guide the value of $\Sigma_{x}$ measured in beam-beam scans, scaling the horizontal spot size down by $15 \%$ at the HER SLM and repeating the beam-beam correction procedure yields $\sigma_{x \mathcal{L}}=66 \mu \mathrm{m}, \Sigma_{x}=143 \mu \mathrm{m}$ and $\Sigma_{y}=7.7 \mu \mathrm{m}$. While the corresponding electron emittance is typical of the values reported in Sec. 9 below, the value of $\Sigma_{x} \times \Sigma_{y} \sim 1100 \mu \mathrm{m}^{2}$ remains incompatible with those extracted from the specific luminosity or the beam-beam scan.

This persistent discrepancy is presumably due to at least one of the vertical IP spot sizes not being accurately predicted using the combination of lattice-function and profile-monitor measurements. The most likely cause of this inconsistency is the sensitivity of the ring optics to the stability of the orbit, especially in the LER. Already at moderate beam currents of a few hundred $\mathrm{mA}$, the ring orbits are significantly different, because of the synchrotron-radiation heat load, from those recorded during lattice measurements, when only one bunch is circulating and the total current is less than a thousandth of its nominal value. The interpretation of beam-profile measurements in terms of IP beam sizes thus becomes very delicate.

Table 6

Comparison of convoluted IP beam sizes determined by various methods. The profile-monitor results are computed from the IP parameters in the bottom rows of Table 5. The bunch lengths assumed in computing the hourglass corrections are $\sigma_{z+}\left(\sigma_{z-}\right)=10.2(9.8) \mathrm{mm}$.

\begin{tabular}{|c|c|}
\hline \hline \multicolumn{2}{|c|}{ Synchrotron-light profile monitors } \\
\hline$\sigma_{x \mathcal{L}}$ & $72.3 \mu \mathrm{m}$ \\
$\sigma_{y \mathcal{L}}\left(z=z_{y}^{w}\right)$ & $3.9 \mu \mathrm{m}$ \\
$\Sigma_{x}$ & $173 \mu \mathrm{m}$ \\
$\Sigma_{y}\left(z=z_{y}^{w}\right)$ & $8.0 \mu \mathrm{m}$ \\
$\Sigma_{x} \times \Sigma_{y}$ & $1387 \mu \mathrm{m}^{2}$ \\
\hline \hline \multicolumn{2}{|c|}{ Beam-beam scans } \\
\hline$\Sigma_{x}, \Sigma_{y}$ (measured) & $150,6.87 \mu \mathrm{m}$ \\
$\Sigma_{y}$ (hourglass corr'd) & $5.24 \mu \mathrm{m}$ \\
$\Sigma_{x} \times \Sigma_{y}$ & $787 \mu \mathrm{m}^{2}$ \\
\hline \hline \multicolumn{2}{|c|}{ Specific luminosity } \\
\hline $\mathcal{L}_{s p}$ (measured) & $4.32 \mu \mathrm{b}^{-1} s^{-1} \mathrm{~mA}^{-2} \mathrm{~b}$ \\
$\mathcal{L}_{s p}$ (hourglass corr'd) & $5.24 \mu \mathrm{b}^{-1} s^{-1} \mathrm{~mA}^{-2} \mathrm{~b}$ \\
$\Sigma_{x} \times \Sigma_{y}$ & $869 \mu \mathrm{m}^{2}$ \\
\hline
\end{tabular}

Numerous attempts to extend this profilemonitor analysis to the strong beam-beam con- 

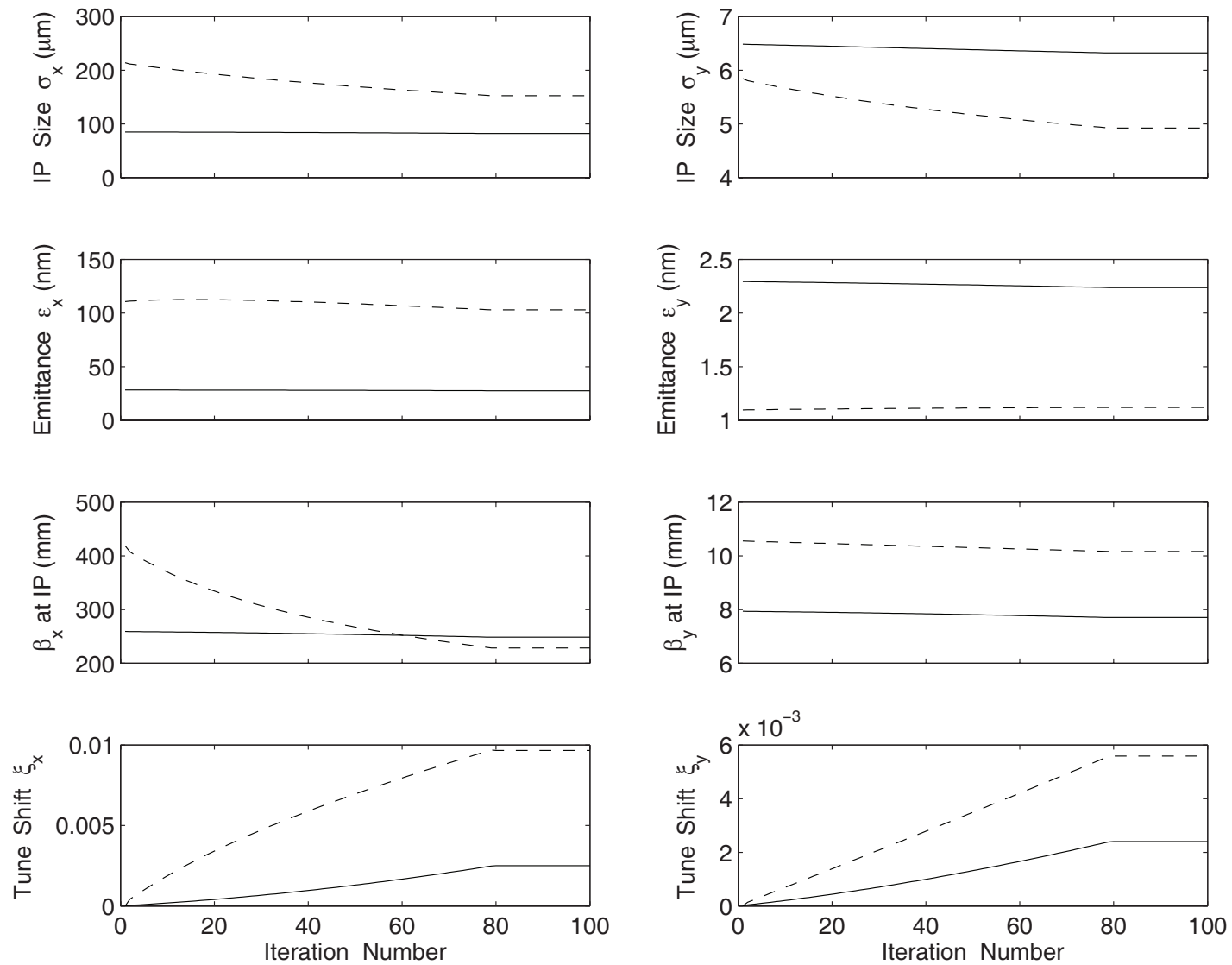

Figure 50. Iterative determination of IP parameters from profile monitor data. The HER transverse beam sizes, emittances, $\beta$-functions and beam-beam parameters are shown by dashed lines; the corresponding LER quantities are the solid lines. The bunch populations are ramped from 0 to their full value in 80 iterations, and then remain constant for an additional 20 .

ditions of routine operating currents have yielded no satisfactory solution. The thermally-induced orbital and optical distortions are clearly exacerbated by the larger heat load. There also exist other reasons why the low-current lattice characterization may not apply under nominal colliding conditions. The low-current data presented above were recorded immediately before the lattice was measured, so at that particular time the state of the machine was as close as could be to the MIAbased model used in the profile-monitor analysis. When PEP-II is operating for physics, the operators continuously tune the machine for maximum luminosity. The procedure includes not only tune adjustments (which have been approximately incorporated into the model), but also changes to 
skew quadrupole settings to optimize the coupling corrections, and to local bumps in sextupoles that affect both coupling and dispersion. Modeling this empirically optimized machine with sufficient precision becomes an intractable proposition.

\section{Combined IP Phase-Space Analysis}

\subsection{Analysis strategy}

The strategy is outlined in Fig. 51, which summarizes the logical flow of the luminous-region analyses and profile-monitor studies described in the preceding sections and consolidated below. Each set of observables: the combination of measured lattice functions and transverse beam profiles on the one hand (Fig. 51, top left), and the luminous-region parameters on the other (Fig. 51, right), offers a nearly complete description of the IP phase space - at least conceptually. Comparing results for overlapping parameters can be used to identify inconsistencies (as illustrated in Sec. 8) or to validate the techniques (see Secs. 9.29.4 below). Combining all measurements should eventually yield a complete, and partially constrained, description.

Transverse-beam profiles at synchrotron-light monitors can be combined with lattice functions measured by resonant excitation, to extract the eigenmode emittances $\epsilon_{1,2}$ in each ring separately, and predict the $e^{+}$and $e^{-}$spot sizes at the IP, and from there the specific luminosity. These traditional measurements can then, in principle, be confronted with luminous-region observables, either as a consistency check or to provide additional constraints. In PEP-II however, the inconsistencies between lattice functions measured in single-bunch mode, and those in collision at intermediate or full beam currents (Sec. 8), significantly restrict the usefulness of profile monitors for phase-space determination at the IP.

The longitudinal and vertical dependence of the luminosity $\mathcal{L}$ and of the angular distribution of the boost vector allow the determination, under high-luminosity conditions, of the convoluted bunch length $\Sigma_{z}$ and of the vertical hourglass parameters (common effective IP $\beta$-function, longitudinal position of the waist, $\sigma_{y \mathcal{L}}$ at the waist). The mutual consistency between the results of these very different methods, and in particular the potential impact of $x-y$ coupling on these analyses, are examined in Sec. 9.2. The impactparameter and transverse-boost techniques also provide, respectively, a constraint on, and a measurement of, the vertical phase-space of the two beams (Sec. 9.3).

The combination of these vertical $e^{ \pm}$IP parameters with the specific luminosity $\mathcal{L}_{s p}$, measured using the radiative-Bhabha monitor, yields the horizontal convoluted beam size $\Sigma_{x}$. Measuring simultaneously $\Sigma_{x}$ and the horizontal luminous size $\sigma_{x \mathcal{L}}$ is tantamount to determining the two $e^{ \pm}$IP horizontal beam sizes (up to a two-fold ambiguity). Finally, the horizontal-boost distribution $\sigma_{x_{\mathrm{B}}^{\prime}}$ directly measures the horizontal $e^{-}$ angular divergence, which together with the horizontal $e^{-}$spot size allows one to solve for $\beta_{x-}^{*}$ and $\epsilon_{x-}$ (Sec 9.4).

The convoluted beam sizes $\Sigma_{x}, \Sigma_{y}$ can also be directly measured by transverse beam-beam scans (Fig. 51, bottom left), providing a partial consistency check on the results above. However, because such scans are possible only in the low beam-beam parameter regime, they reflect neither the full impact of the horizontal dynamic- $\beta$ effect, nor that of beam-beam-induced verticalemittance blowup. The values of $\Sigma_{x}$ and $\Sigma_{y}$ extracted from such scans therefore represent an upper and a lower limit, respectively, of the corresponding IP spot-size combinations under highluminosity conditions.

\subsection{Vertical hourglass parameters}

The long-term evolution of the effective vertical IP $\beta$-function is presented in Fig. 52 . The results of the longitudinal luminosity profile and boost methods are highly consistent (as predicted by simulations), typically tracking each other within $1 \mathrm{~mm}$. The vertical luminous-size results display, over most of the running period, relative variations similar to those of the other two methods, but with $\beta_{y}^{*}$ values lower by 3 to $5 \mathrm{~mm}$ and more consistent with phase advance measurements (see Fig. 25).

The corresponding history of the vertical-waist positions are shown in Fig. 53. Here again, the relative variations in $z_{y}^{w}$ are highly corre- 


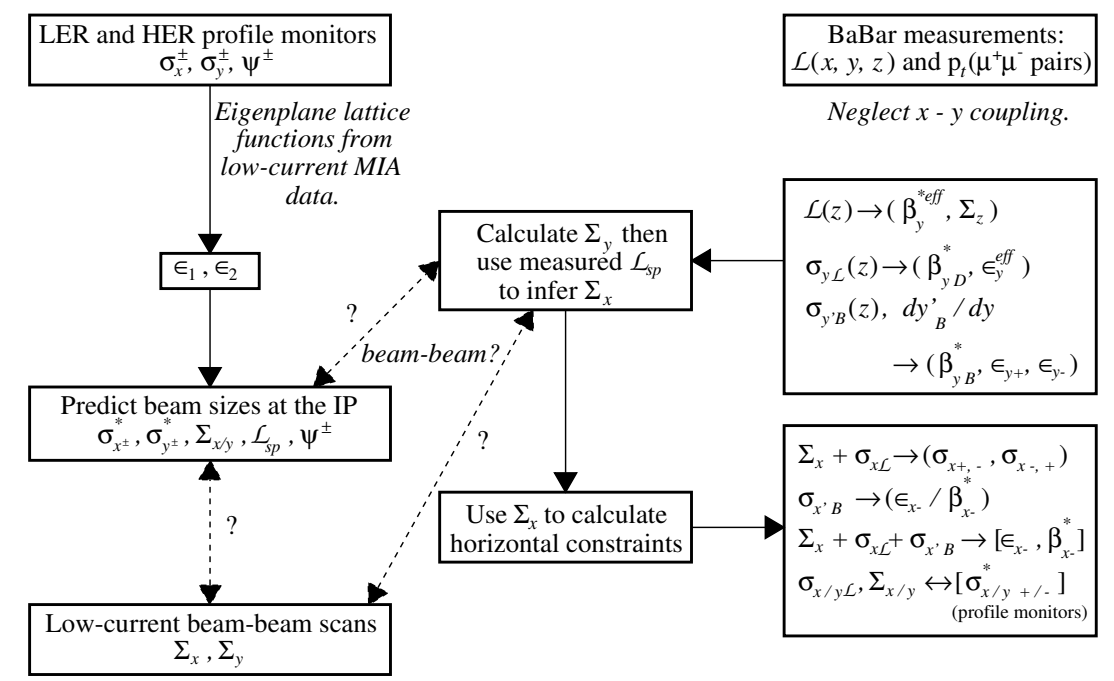

Figure 51. Schematic of possible inputs to a combined analysis of the two-beam phase space at the IP. Variables within parentheses are obtained from the indicated measurements, whereas variables within square brackets can be constrained by, but not fully determined from, the indicated measurements.

lated, suggesting they reflect actual waist displacements. The boost method provides the most statistically precise measurement; the reported waist typically lies within $1 \mathrm{~mm}$ of the longitudinal position $z_{c}$ of the collision point (which is extracted independently from the longitudinal luminosity distribution). The systematic difference in waist position between the longitudinal luminosity profile and the boost method could not be explained by instrumental, acceptance, or beambeam effects. It appears consistent in sign and magnitude with the impact, on the longitudinal luminosity distribution, of an impedance-induced asymmetry in the longitudinal positron distribution (already indicated by streak-camera measurements [26]). But this difference in the reported position of the effective waist may also be due to separated $e^{+}$and $e^{-}$waists, or to different sensitivities of the two methods to $x-y$ coupling. However it remains small enough $(\sim 2 \mathrm{~mm}$, i.e. $15 \%$ of $\beta_{y}^{*}$ ) not to bias the $\beta_{y}^{*}$ measurement.

Although none of the luminous-region methods by itself is able to determine separately $\beta_{y+}^{*}$ and $\beta_{y-}^{*}$, each of these measurements imposes a different constraint on the allowed values of the two IP $\beta$-functions; requiring that their results overlap, may therefore restrict the range of allowed $\beta_{y+,-}^{*}$ values. These constraints are weakly dependent on the vertical $e^{+}$and $e^{-}$emittances, and either can be expressed analytically (see Eq. 10 for the longitudinal luminosity distribution), or are computed numerically (vertical luminous-size and boost methods). An example of the resulting contours is presented in Fig. 54. The boost and longitudinal-profile constraints mostly overlap, but the luminous-size method provides orthogonal information. However, the overlap region $\left(\beta_{y+}^{*} \sim 9 \mathrm{~mm}, \beta_{y-}^{*} \sim 21 \mathrm{~mm}\right)$ is inconsistent both with single-bunch phase advance data (open circles), and with predictions from PEP-II lattice models.

This inconsistency could not be explained by systematic instrumental or acceptance biases, nor by beam-beam distortions; neither could it be resolved by reasonable variations of the assumed emittances. To investigate the potential impact 

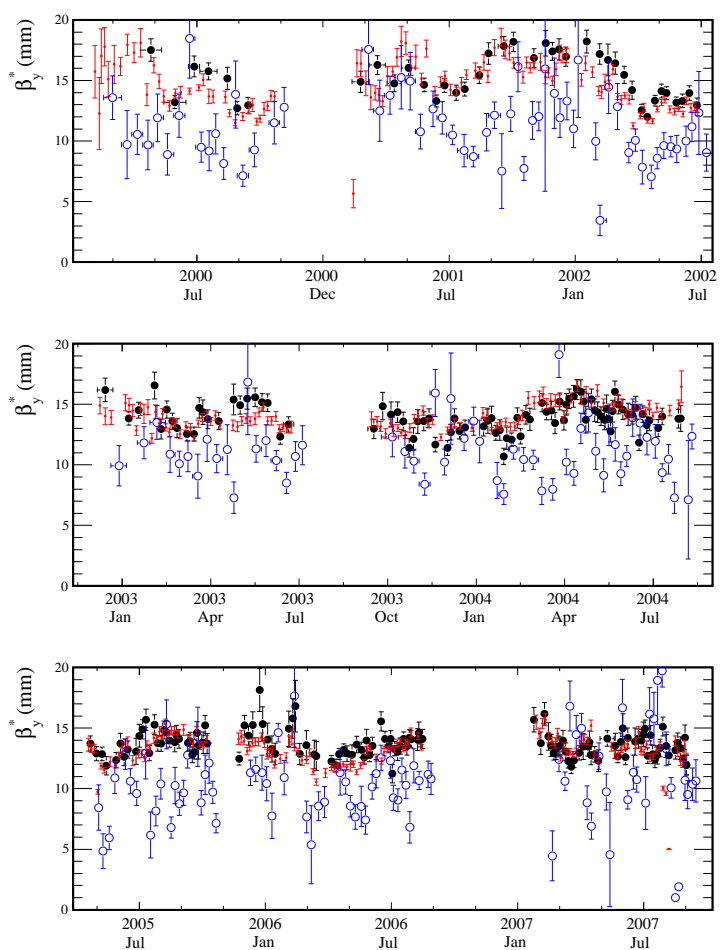

Figure 52. History of the vertical IP $\beta$-function, measured using the longitudinal luminosity profile $\left(\beta_{y \text {,eff }}^{*}\right.$, small red squares), the RMS angular spread of the $\mu^{+} \mu^{-}$boost vector $\left(\beta_{y, B}^{*}\right.$, full black circles), and the vertical luminous size $\left(\beta_{y, D}^{*}\right.$, open blue circles).

of $x$-y coupling, simulated luminosity and boostangle distributions were generated using sets of lattice functions measured, over the course of one year, in single-bunch mode by the MIA method (Sec. 3.6). Applying the (uncoupled) analyses of Secs. 5, 6 and 7 to these simulated distributions yields the results displayed in Fig. 55. Here each symbol shows the results of the fit to a data set that was generated assuming either coupling as measured (squares and circles), or with coupling turned off (triangles) by setting the $w$ matrix (Eqs. 17, 18) to zero. In all cases, the coupling distorts the hourglass shape, as evidenced by the
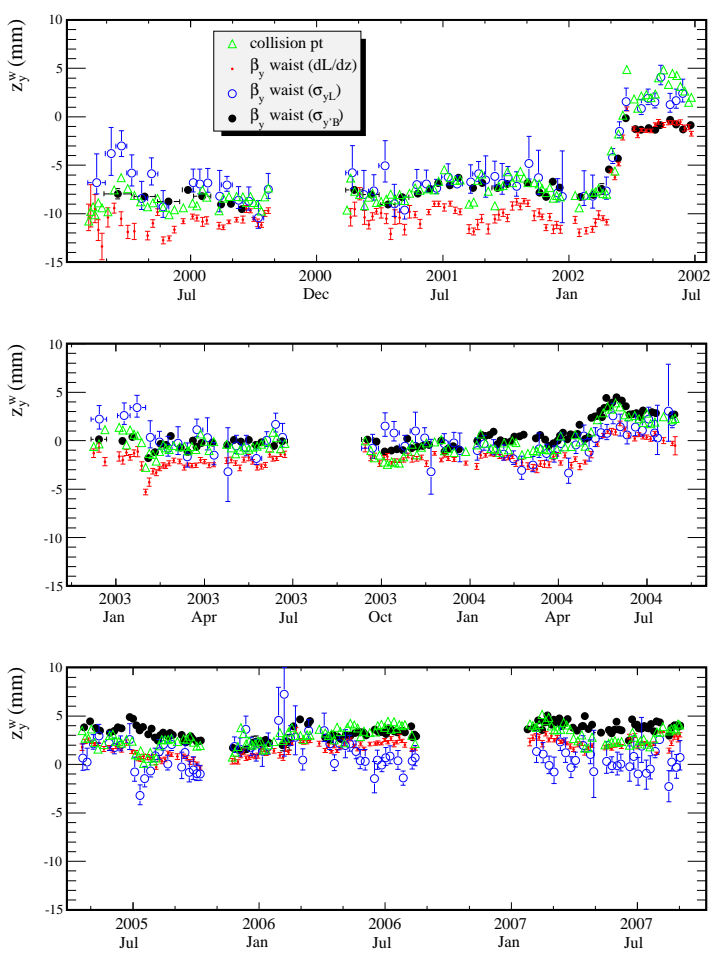

Figure 53. History of the longitudinal position of the (assumed common) vertical waist, measured using the longitudinal luminosity profile (small red squares), the angular spread of the $\mu^{+} \mu^{-}$ boost vector (full black circles), and the vertical luminous size (open blue circles). The green triangles are the fitted position $z_{c}$ of the collision point.

correlation, across the three analyses, of sampleto-sample variations. The $\beta_{y}^{*}$ bias is smallest $(0.5-2 \mathrm{~mm})$ for the luminous-size method, and largest $(1-5 \mathrm{~mm})$ for the boost technique.

A similar analysis was applied to the measurements of the vertical luminous size at the waist, displayed in Fig. 56. Remarkably, the impactparameter technique and the boost method - fundamentally different as they are - yield results consistent to better than $1 \mu \mathrm{m}$ RMS. A systematic difference is however apparent, which would 


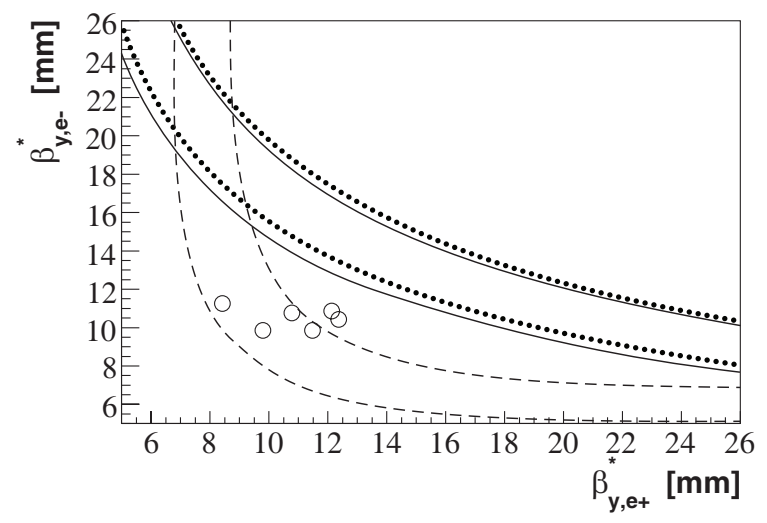

Figure 54. Constraints in the $\beta_{y+}^{*}-\beta_{y-}^{*}$ plane extracted from three months of BABAR data (July to September 2005), by different methods. Dotted line: longitudinal luminosity profile $\left(\beta_{y, \text { eff }}^{*}\right.$ $=14 \pm 1 \mathrm{~mm}$ ). Solid line: angular spread of the boost vector $\left(\beta_{y, B}^{*}=14 \pm 1 \mathrm{~mm}\right)$. Dashed line: vertical luminous size $\left(\beta_{y, D}^{*}=10 \pm 1 \mathrm{~mm}\right)$. These lines are $\pm 1 \sigma$ contours, drawn assuming $\epsilon_{y-}\left(\epsilon_{y+}\right)=3(2)$ nm-rad as suggested by Fig. 43. The open circles represent low-current, phaseadvance measurements of $\beta_{y+,-}^{*}$ that assume no $x-y$ coupling.

be consistent, both in sign and in magnitude, with underestimating the bias correction (Sec. 6.4.3) to the luminous size by roughly $1 \mu \mathrm{m}$. In contrast, a simulation study of coupling-induced biases, summarized in Fig. 57, suggests that the impact of transverse coupling on the measured vertical luminous size is typically larger when using the boost rather than the impact-parameter method (as was also the case for $\beta_{y}^{*}$ ). It may well be, therefore, that the systematic difference observed in Fig. 56 is actually associated with an underestimation of either the $e^{+}$or $e^{-}$vertical emittance by the boost analysis.

\subsection{Vertical Phase Space}

Measurements of the vertical $e^{+}$and $e^{-}$ emittances by the boost method, assuming $\beta_{y+}^{*}=\beta_{y-}^{*}$, were discussed in Sec. 7.2.3 and
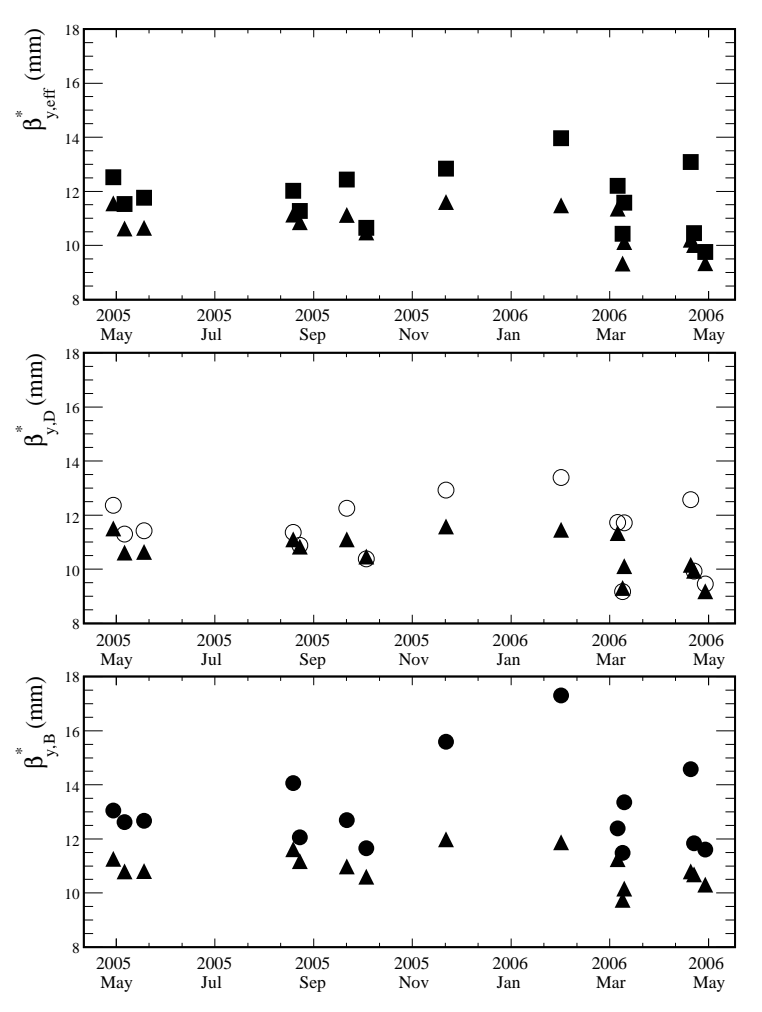

Figure 55. Impact of coupling on simulated $\beta_{y}^{*}$ measurements by different methods: longitudinal luminosity profile ( $\beta_{y \text {,eff }}^{*}$, top); vertical luminous size $\left(\beta_{y, D}^{*}\right.$, center $)$; vertical-boost angular spread $\left(\beta_{y, B}^{*}\right.$, bottom $)$. The symbols are defined in the text.

presented in Fig. 43. Simulations indicate that in the absence of transverse coupling and because of the energy asymmetry, the HER vertical emittance is determined more reliably than the LER emittance. In addition, and due to internal correlations, the fitted vertical $e^{+}$IP spot size $\left(\sqrt{\epsilon_{y+} \times \beta_{y, B}^{*}}\right)$ is less sensitive to unequal $e^{+}-e^{-}$ $\beta$-functions and emittances, than the corresponding angular spread $\left(\sqrt{\epsilon_{y+} / \beta_{y, B}^{*}}\right)$ or than the LER emittance itself.

The boost measurements are confronted with the impact-parameter results in Fig. 58, using the effective vertical emittance defined in Eq. 11. The 

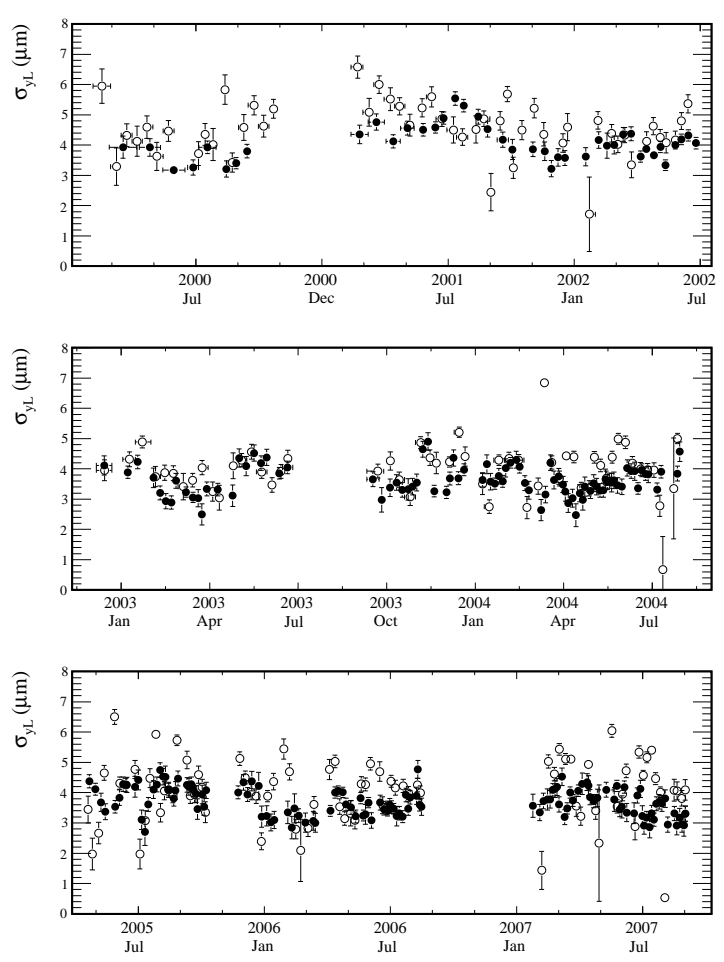

Figure 56. History of the vertical luminous size $\sigma_{y \mathcal{L}}$, directly measured by the impact-parameter method (open circles), or inferred from the results $\left(\epsilon_{y+}, \epsilon_{y-}, \beta_{y, B}^{*}\right)$ of the boost analysis (full circles).

two techniques track each other nicely in terms of relative variations, suggesting these reflect actual changes in the vertical emittance(s). The difference between emittances measured by the impact-parameter and by the boost method has the same sign as, but is more pronounced than in the case of the vertical luminous-size determination (Fig. 56). Part of the discrepancy might be due, as in the case of $\sigma_{y \mathcal{L}}$, to an underestimated bias correction $\left(\sigma_{y \mathcal{L}}, \beta_{y, D}^{*}\right.$ and $\epsilon_{y \text {,eff }}$ all decrease when the bias correction increases). But an additional contribution to the discrepancy in vertical effective emittance may be attributable to the systematic difference (most likely caused by $x$ $y$ coupling) between the effective $\beta$-functions re-

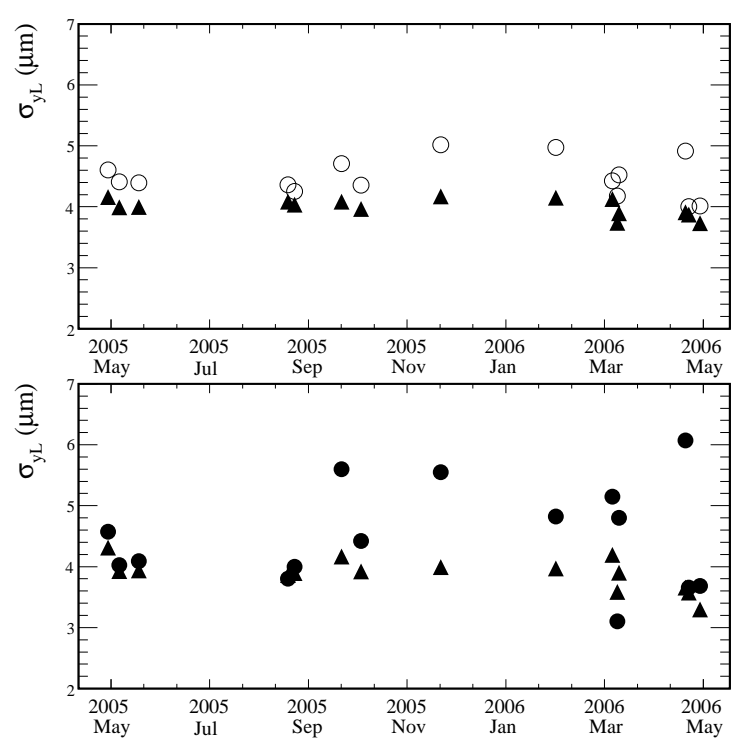

Figure 57. Impact of coupling on simulated $\sigma_{y \mathcal{L}}$ measurements by the impact-parameter (top) and by the boost (bottom) methods. Each symbol shows the results of the fit to simulated luminosity and boost-angle distributions generated using one set of lattice functions measured in singlebunch mode at the indicated date, and assuming either zero coupling (triangles), or coupling as measured (open circles, full circles).

turned by the boost and impact-parameter methods $\left(\beta_{y, B}^{*}>\beta_{y, D}^{*}\right.$, see Fig. 52$)$.

Figure 59 illustrates the potential impact of transverse coupling on simulated vertical emittance and $\beta_{y}^{*}$ measurements by the boost method. Boost angular distributions were generated, using the same sets of measured lattice functions as in the preceding section and assuming $\epsilon_{y+}=\epsilon_{y-}=3 \mathrm{~nm}$-rad. In the absence of $x-y$ coupling, the fitted effective $\beta$-function $\beta_{y, B}^{*}$ (triangles, top) and HER emittance (triangles, center) perfectly reproduce the corresponding input parameter (crosses); however the fitted LER emittance (triangles, bottom) deviates from its generated value, the more so the more the true value of $\beta_{y-}^{*}$ differs from that of $\beta_{y+}^{*}$. When coupling 

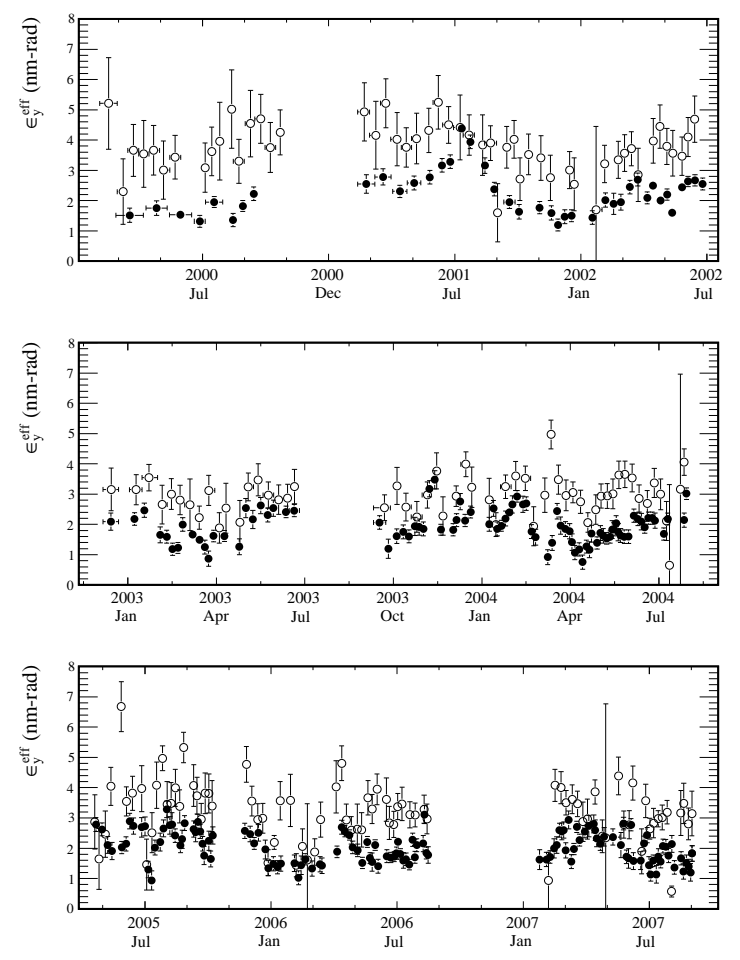

Figure 58. History of the effective vertical emittance $\epsilon_{y \text {,eff }}$, measured using the impact-parameter method (open circles), or inferred from the results $\left(\epsilon_{y+}, \epsilon_{y-}, \beta_{y, B}^{*}\right)$ of the boost analysis (full circles).

is turned on (circles), the fitted value of $\beta_{y, B}^{*}$ typically increases, and the fitted electron and positron emittances both deviate from their true value. Further simulations have shown that these emittance biases can be of either sign, depending on the coupling parameters and on the relative true values of $\beta_{y+}^{*}, \beta_{y-}^{*}, \epsilon_{y-}$ and $\epsilon_{y+}$.

\subsection{Horizontal phase space}

In spite of the systematic uncertainties introduced by residual coupling near the IP, a partial validation of the vertical phase-space measurements above is however provided by the comparison (Fig. 60, top) of the directly-measurable horizontal luminous size $\sigma_{x \mathcal{L}}$ (Eq. 8) with the hor-
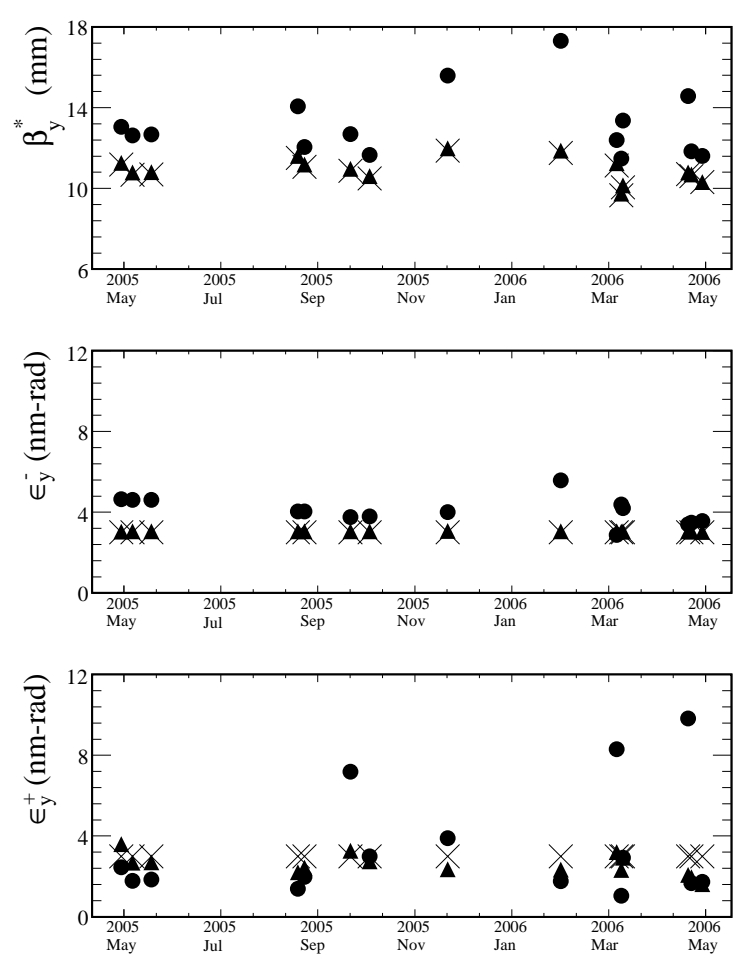

Figure 59. Impact of coupling on simulated vertical phase-space measurements by the boost method: IP $\beta$-function $\beta_{y, B}^{*}$ (top), $e^{-}$(center) and $e^{+}$(bottom) emittances. The crosses are the true (input) values. The other symbols show the results of the fit to simulated boost-angle distributions generated using lattice functions measured in single-bunch mode, and assuming either zero coupling (triangles), or coupling as measured (full circles).

izontal convoluted beam size $\Sigma_{x}$ (Eq. 7). The latter is calculated from the IP vertical $\beta$-functions (Fig. 42) and emittances (Fig. 43) extracted from the boost distribution, and from the measured specific luminosity $\left(\mathcal{L}_{s p} \sim 1 / \Sigma_{x} \Sigma_{y}\right)$ averaged over the corresponding time window and hourglass-corrected [10] using the measured ratio of $\beta_{y, B}^{*} / \Sigma_{z}$. The ratio $\Sigma_{x} / \sigma_{x \mathcal{L}}$ (Fig. 60 , bottom) is determined by that of the $e^{+}$and $e^{-}$hor- 
izontal beam sizes, and reaches a minimum of 2 when $\sigma_{x-}=\sigma_{x+}$. The range of observed values is consistent with this physical bound and provides an indication of the relative horizontal beam sizes under the simplifying assumption of negligible coupling. The same data can also be presented in terms of a determination of the two horizontal IP beam sizes (Fig. 61). Additional input is required to lift the ambiguity as to which of the two beams exhibits the largest horizontal spot size at the IP.
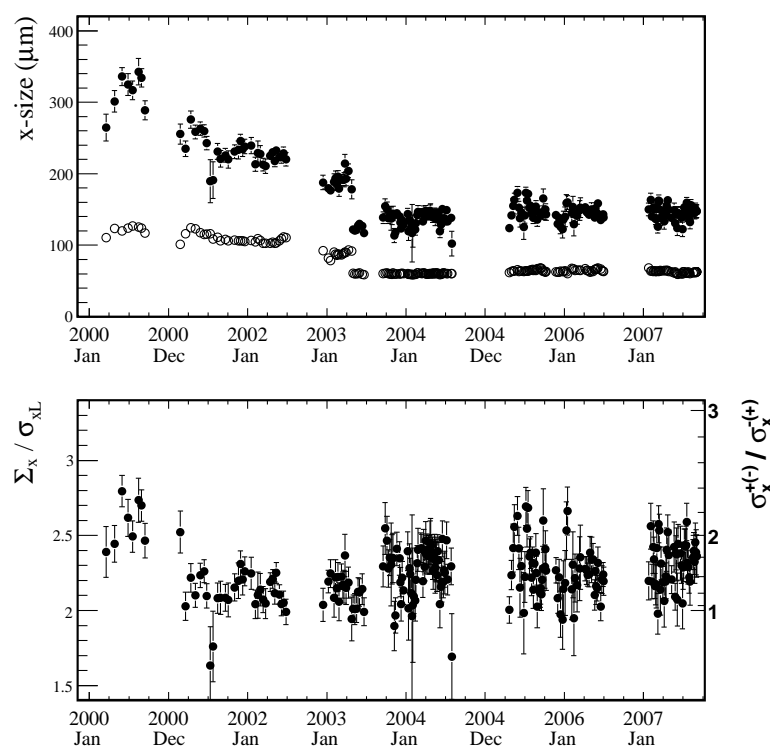

Figure 60. Top: comparison of $\Sigma_{x}$ (points with error bars), inferred from the measured specific luminosity and the vertical-boost analysis, with the horizontal luminous size $\sigma_{x \mathcal{L}}$ (open circles). Their ratio (bottom, left scale) is determined by that of the $e+$ and $e^{-}$horizontal beam sizes (bottom, right scale).

One more handle is provided by the horizontalboost angular spread, which (up to a $\sim 6 \%$ correction) is dominated by the HER angular divergence (Eq. 13). Combining $\sigma_{x_{-}^{\prime}}$ with the possible

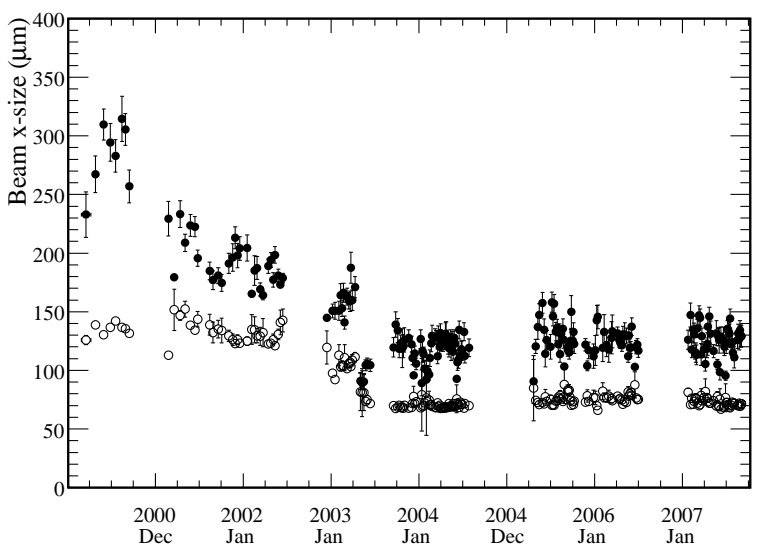

Figure 61. Seven-year history of the $e^{-}$and $e^{+}$horizontal IP spot sizes, inferred by combining $\Sigma_{x}$ and $\sigma_{x \mathcal{L}}$ measurements. Luminous-region analyses do not allow, by themselves, to lift the ambiguity. The step-like drop in mid 2003 occurred when the horizontal tunes of both rings were moved very close to the half-integer.

solutions for $\sigma_{x_{-}}$yields two solutions for the horizontal emittance and IP $\beta$-function of the electron beam, presented in Fig. 62. Recent models of the PEP-II optics tuned on low-current lattice measurements [38], consistently suggest that the horizontal LER emittance is the smallest of the two, thereby lifting the ambiguity in Fig. 62 . This in turn identifies the $e^{+}$horizontal IP spot size as the smaller of the two (open circles) in Fig. 61. These lattice models predict $\epsilon_{x-}\left(\epsilon_{x+}\right)=50-60$ (20-40) nm, yielding a reasonable agreement with the high-current measurement of $\epsilon_{x-}$ presented here.

\section{Conclusions}

We presented in this paper the development and application of a novel suite of analysis tools that extract an extensive set of $e^{+}$and $e^{-}$beam parameters at the PEP-II IP, from the spatial and kinematic distributions of $e^{+} e^{-} \rightarrow \mu^{+} \mu^{-}$events reconstructed using the $B A B A R$ detector. 

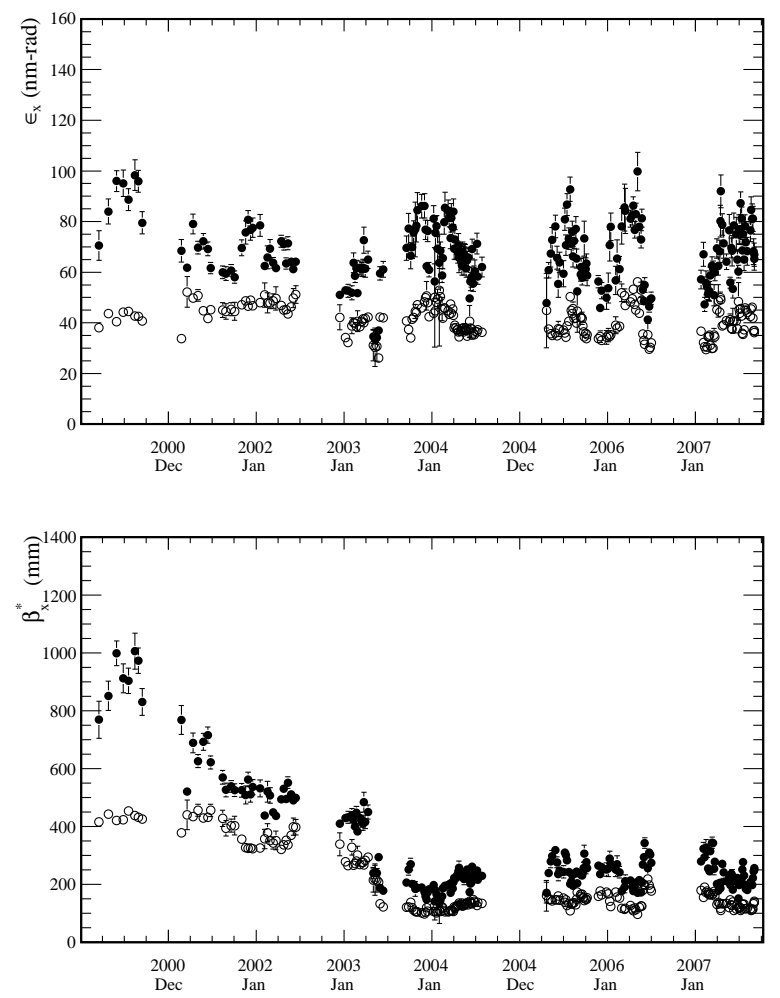

Figure 62. Seven-year history of the $e^{-}$horizontal emittance (top) and IP $\beta$-function (bottom), inferred by combining $\Sigma_{x}, \sigma_{x \mathcal{L}}$ and $\sigma_{x_{\mathrm{B}}^{\prime}}$ measurements. Open circles correspond to the $\sigma_{x-}<\sigma_{x+}$ solution, full circles to $\sigma_{x-}>\sigma_{x+}$. Simulations of the PEP-II lattice favor the latter.

While monitoring the luminous-centroid position is by now routine procedure in most colliding-beam experiments, the high luminosity of $e^{+} e^{-}$factories allows in addition the continuous determination, on much shorter time scales, of the orientation of the luminous ellipsoid with respect to the nominal beam axis. In the special case of the $B$ factories, the $e^{+}-e^{-}$energy asymmetry also gives access to the horizontal and vertical crossing angles between the two beams, because that asymmetry is reflected in the transverse-momentum distributions of fully- reconstructed final states.

More importantly, the simultaneous analysis of the three-dimensional luminosity distribution and of the angular distribution of muon pairs allows a continuous determination, under normal highluminosity conditions and on time scales varying from a half hour to a few weeks, of a large panoply of longitudinal and transverse phase-space parameters. The use of three complementary techniques, with very different instrumental systematics and diverse sensitivities to the unknown beam parameters, allows, in some cases, to separate electron and positron properties; it also provides much-needed redundancy and allows crucial consistency checks. Several of the observables accessible to these luminous-region analyses are either not directly measurable by conventional accelerator techniques, or can be determined only at very low current or in single-bunch mode, greatly complicating the interpretation of accelerator performance during physics running.

The list of the measured phase-space and optics parameters studied in this paper is presented in Table 7 , which constitutes a self-consistent snapshot of beam properties at the PEP-II IP at the end of the 2007 run. The long-term evolution of these quantities, measured over the entire life of the SLAC $B$-factory, was presented in Secs. 5, 7 and 9 . These history plots demonstrate the progressive optimization of the optics and emittances of each of the beams, and provide striking illustrations of, for instance, the onset of the dynamic- $\beta$ effect.

The techniques presented in this paper are in principle applicable to all high-luminosity $e^{+} e^{-}$ colliders - albeit to various degrees. In particular, the full potential of the very powerful boost technique can only be realized in the presence of a significant asymmetry between beam energies. The impact-parameter method may run into fundamental instrumental limitations in some of the proposed "super-B" factories, where sub-micron IP spot sizes can no longer be resolved by vertex detectors. Except for the boost technique (which requires fully-reconstructed exclusive final states), the methods introduced here should also prove useful in hadron colliders. 
Table 7

Typical beam parameters at the PEP-II IP extracted from luminous-region measurements in August 2007. Errors are statistical only. The longitudinal phase-space measurements were presented in Sec. 5, and the impact-parameter (DOCA) and boost (B) methods detailed in Secs. 6 and 7 respectively. Combined analyses of transverse phase space were discussed in Sec. 9. The dominant systematic uncertainty affecting vertical parameters is indicated in the right column; in most cases it also impacts the derived horizontal parameters.

\begin{tabular}{|c|c|c|c|c|c|}
\hline IP parameter & $e^{-}$ & & $e^{+}$ & Method & Comments \\
\hline $\begin{array}{l}\text { Total beam current }(\mathrm{A}) \\
\text { Number of bunches } \\
\text { Bunch current }(\mathrm{mA} / \mathrm{b}) \\
\text { Luminosity }\left(\mathrm{cm}^{-2} s^{-1}\right) \\
\mathcal{L}_{s p}\left(\mathrm{~cm}^{-2} s^{-1} \mathrm{~b}^{-1}(\mathrm{~mA} / \mathrm{b})^{-2}\right)\end{array}$ & $\begin{array}{l}1.796 \\
1722 \\
1.04\end{array}$ & $\begin{array}{l}11.1 \times 10^{33} \\
3.98 \times 10^{30}\end{array}$ & $\begin{array}{l}2.526 \\
1722 \\
1.47\end{array}$ & $\begin{array}{l}\text { rad. Bhabha } \\
\text { rad. Bhabha }\end{array}$ & $\begin{array}{l}\text { bef. hourglass crct'n } \\
\text { bef. hourglass crct'n }\end{array}$ \\
\hline $\begin{array}{l}\Sigma_{z}(\mathrm{~mm}) \\
\sigma_{z}(\mathrm{~mm}) \\
\end{array}$ & $11.1 \pm 1.1$ & $\overline{15.15 \pm 0.02}$ & $11.1 \pm 1.1$ & $\begin{array}{c}\mathcal{L}(z) \\
\mathcal{L}(z) \& V_{R F} \\
\end{array}$ & Opportunistic only \\
\hline $\begin{array}{l}\beta_{y, D}^{*}(\mathrm{~mm}) \\
\beta_{y, \text { eff }}^{*}(\mathrm{~mm}) \\
\beta_{y, B}^{*}(\mathrm{~mm}) \\
\epsilon_{y, \text { eff }}(\mathrm{nm}-\mathrm{rad}) \\
\epsilon_{y, \text { eff }}(\mathrm{nm}-\mathrm{rad}) \\
\epsilon_{y-,+}(\mathrm{nm}-\mathrm{rad}) \\
\sigma_{y-},+(\mu \mathrm{m}) \\
\Sigma_{y}(\mu \mathrm{m}) \\
\sigma_{y \mathcal{L}}(\mu \mathrm{m}) \\
\sigma_{y \mathcal{L}}(\mu \mathrm{m}) \\
\sigma_{y}\end{array}$ & $\begin{array}{l}2.5 \pm 0.1 \\
5.7 \pm 0.3\end{array}$ & $\begin{array}{c}10.1 \pm 0.8 \\
12.8 \pm 0.2 \\
12.9 \pm 0.3 \\
3.1 \pm 0.4 \\
1.5 \pm 0.1 \\
\\
6.9 \pm 0.2 \\
3.2 \pm 0.1 \\
4.0 \pm 0.2 \\
\end{array}$ & $\begin{array}{l}1.0 \pm 0.1 \\
3.6 \pm 0.2\end{array}$ & $\begin{array}{c}\text { DOCA } \\
\mathcal{L}(z) \\
\mathrm{B} \\
\text { DOCA } \\
\epsilon_{y+} \& \epsilon_{y-(\mathrm{B})} \\
\mathrm{B} \\
\mathrm{B} \\
\sigma_{y+,-}(\mathrm{B}) \\
\sigma_{y+,-}(\mathrm{B}) \\
\text { DOCA } \\
\end{array}$ & $\begin{array}{l}\text { Resolution bias } \\
\text { Coupling } \\
\text { Coupling } \\
\text { Resolution bias } \\
\text { Coupling } \\
\text { Coupling } \\
\text { Coupling } \\
\\
\text { Resolution bias } \\
\end{array}$ \\
\hline $\begin{array}{l}\sigma_{x \mathcal{L}}(\mu \mathrm{m}) \\
\Sigma_{x}(\mu \mathrm{m}) \\
\sigma_{x-,}(\mu \mathrm{m}) \\
\sigma_{x-}^{\prime}(\mathrm{mrad}) \\
\epsilon_{x-}(\mathrm{nm}-\mathrm{rad}) \\
\beta_{x-}^{*}(\mathrm{~mm})\end{array}$ & $\begin{array}{c}129 \pm 4 \\
0.60 \pm 0.02 \\
75 \pm 2 \\
222 \pm 6\end{array}$ & $\begin{array}{c}64.0 \pm 0.1 \\
148 \pm 3\end{array}$ & $\begin{array}{c}71 \pm 1 \\
- \\
- \\
-\end{array}$ & $\begin{array}{c}\text { DOCA } \\
\mathcal{L}_{s p} \& \Sigma_{y} \\
\Sigma_{x} \& \sigma_{x} \mathcal{L} \\
\text { B } \\
\sigma_{x} \& \sigma_{x}^{\prime} \\
\sigma_{x} \& \sigma_{x}^{\prime}\end{array}$ & \\
\hline
\end{tabular}

\section{Acknowledgments}

The work reported here has greatly benefited from the insight, encouragement and intellectual challenges from our accelerator colleagues over the last several years: we would like to thank, in particular, S. Ecklund, J. Seeman, M. Sullivan, U. Wienands and Y. Yan. We are grateful for the excellent luminosity and machine conditions provided by the PEP-II Operations team, as well as for the dedicated efforts by our BABAR collaborators and by the computing organizations that support the SLAC $B$ Factory program. The collaborating institutions wish to thank SLAC for its support and kind hospitality. This work is supported by DOE and NSF (USA), NSERC (Canada), CEA and CNRS-IN2P3 (France), BMBF and DFG (Ger- many), INFN (Italy), FOM (The Netherlands), NFR (Norway), MES (Russia), MEC (Spain), and STFC (United Kingdom). Individuals received support from the Marie Curie EIF (European Union) and the A. P. Sloan Foundation.

\section{REFERENCES}

1. D. Cinabro et al., Nucl. Instrum. Meth. A 481, 29 (2002).

2. D. Cinabro et al., Phys. Rev. E57 (1998) 1193.

3. J. Slaughter et al., "Tevatron Run II Luminosity, Emittance and Collision Point", Proc. Particle Accelerator Conference (PAC) 2003, Vol. 3, $1763-1765$.

4. "PEP-II: An Asymmetric B Factory", Conceptual Design Report, SLAC-R-418 (1993).

5. B. Aubert et al. [BABAR Collaboration], Nucl. 
Instrum. Meth. A 479, 1 (2002).

6. U. Wienands et al., SLAC-PUB-12612 (2007).

7. S. Ecklund, C. Field and G. Mazaheri, Nucl. Instrum. Meth. A 463, 68 (2001).

8. P. Billoir, Nucl. Instrum. Meth. A 225, 352 (1984); D. N. Brown, E. A. Charles, and D. A. Roberts, "The BABAR track fitting algorithm," Proceedings of CHEP 2000, Padova, Italy (2000).

9. D. Brown, A. Gritsan and D. Roberts, "Internal alignment of the BABAR silicon vertex tracking detector," First LHC Detector Alignment Workshop, Geneva, Switzerland (2006).

10. M. Venturini and W. Kozanecki, SLAC-PUB8699 (2001).

11. M. Venturini and W. Kozanecki, SLAC-PUB8700 (2001); W. Kozanecki et al., "Beambeam experience at the PEP-II B factory", Proc. International Workshop " $e^{+} e^{-}$Factories 99", Tsukuba, Japan, 21-24 Sep 1999.

12. Y. Cai, SLAC-PUB-8479 (2000).

13. P. Castro-Garcia, "Luminosity and Beta Function Measurement at the ElectronPositron Collider Ring LEP", Doctoral Thesis, Universitat de Valencia, May 1996.

14. Y.T. Yan, "Storage ring measurement, model, and correction", in ICFA Beam Dynamics Newsletter, No. 42, Sec. 4-2, P. 71-87, Y. Cai ed.; and reference therein.

15. Y. Yan et al., SLAC-PUB-11209 (2005).

16. A.S. Fisher, "Instrumentation and Diagnostics for PEP-II", Beam Instrumentation Workshop, Stanford, CA 4-7 May 1998, AIP Conf. Proc. 451 (Amer. Inst, Physics, Woodbury, NY, 1998), pp. 395-403.

17. A.S. Fisher et al., "Design of an X-Ray Imaging System for the Low- Energy Ring of PEP-II", Proc. European Particle Accelerator Conf. 2004, 5-9 July 2004, Lucerne, Switzerland, SLAC-PUB-10528.

18. A. S. Fisher et al., "Design of the Synchrotron-Light Monitors for PEP-II", Proc. European Particle Accelerator Conf., Barcelona, Spain, June 1996, p. 1734.

19. A. S. Fisher et al., "A Synchrotron-Light Interferometer for PEP-II", Proc. Beam Instrumentation Workshop 2000, Cambridge, MA,
8-11 May 2000, SLAC-PUB-8456.

20. A. S. Fisher et al., "Beam-Size Measurements on PEP-II Using Synchrotron-Light Interferometry", Proc. Particle Accelerator Conf. 2001, Chicago, IL, 18-22 June 2001, pp. 547549 .

21. A. S. Fisher, "Fringe Pattern of the PEP-II Synchrotron-Light Interferometers", SLACTN-05-048.

22. Y. Cai, "Method of measuring the coupled lattice functions at the interaction point in $e^{+} e^{-}$storage rings", Phys. Rev. E 68036501 (2003).

23. Y. Cai et al., SLAC-PUB-7642 (1997).

24. Y. Cai et al., EPAC-2006-MOPLS052.

25. S. Agostinelli et al. [GEANT4 Collaboration], Nucl. Instrum. Meth. A 506, 250 (2003).

26. W. Cheng, A. Fisher, and J. Corbett, "Streak-Camera Measurements with High Currents in Pep-II and Variable Optics in Spear3", contributed to the 13th Beam Instrumentation Workshop (BIW08), Lake Tahoe (CA), May 2008.

27. I. V. Narsky et al., "Study of Beam-beam Effects at PEP-II", Proc. 9th European Particle Accelerator Conference (EPAC 2004).

28. A. Fisher et al., "Bunch-Length Measurements in PEP-II", SLAC-PUB-11230 and Proc. 2005 Particle Accelerator Conference, PAC-2005-TPPP026.

29. A. Novokhatski, private communication (2008).

30. D. Teytelman, private communication (SLAC-PUB in preparation).

31. G. Schott, Ph. D. Dissertation, Universite Paris VI (2004).

32. J. M. Thompson, Ph. D. Dissertation, Stanford University (2008).

33. D. Cinabro et al., CLNS 02/1812 (2002).

34. W. Kozanecki et al., SLAC-PUB-11216 (2005).

35. Y. Funakoshi et al., "Orbit feedback system for maintaining an optimum beam collision", Phys. Rev. ST Accel. Beams 10:101001 (2007).

36. K. Yokoya and P. Chen, "Beam-Beam Interaction in Linear Colliders", in Lecture Notes in Physics No. 400, p. 415, ed. M. Dienes, 
M. Month, and S. Turner (Springer-Verlag, 1992).

37. D. Sagan and D. Rubin, "Linear analysis of coupled lattices", Phys. Rev. ST Accel. Beams 2:074001 (1999).

38. Y. Cai and Y. Yan, private communication; U. Wienands et al., Presentation to the PEP-II Machine Advisory Committee (2007), http://www.slac.stanford.edu/grp/ad/ pep-ii/MAC-Review/MAC-Nov2007/ Wienands_beam-beam_fin.pdf (unpublished) 